\title{
WestVirginiaUniversity
}

THE RESEARCH REPOSITORY @ WVU

Graduate Theses, Dissertations, and Problem Reports

2013

\section{The role of focal adhesion kinase in nonmuscle cell contraction}

Kimberly M. Arnold

West Virginia University

Follow this and additional works at: https://researchrepository.wvu.edu/etd

\section{Recommended Citation}

Arnold, Kimberly M., "The role of focal adhesion kinase in nonmuscle cell contraction" (2013). Graduate Theses, Dissertations, and Problem Reports. 4949.

https://researchrepository.wvu.edu/etd/4949

This Dissertation is protected by copyright and/or related rights. It has been brought to you by the The Research Repository @ WVU with permission from the rights-holder(s). You are free to use this Dissertation in any way that is permitted by the copyright and related rights legislation that applies to your use. For other uses you must obtain permission from the rights-holder(s) directly, unless additional rights are indicated by a Creative Commons license in the record and/ or on the work itself. This Dissertation has been accepted for inclusion in WVU Graduate Theses, Dissertations, and Problem Reports collection by an authorized administrator of The Research Repository @ WVU.

For more information, please contact researchrepository@mail.wvu.edu. 
The role of focal adhesion kinase in nonmuscle cell contraction

Kimberly M. Arnold

\begin{abstract}
Dissertation submitted to the School of Medicine at West Virginia University

in partial fulfillment of the requirements

for the degree of
\end{abstract}

Doctor of Philosophy

in

Cellular \& Integrative Physiology

\author{
Robert Wysolmerski, Ph.D., Chair \\ Jun Liu, Ph.D. \\ Karen Martin, Ph.D. \\ Fred Minnear, Ph.D. \\ Michael Schaller, Ph.D. \\ Department of Physiology \& Pharmacology
}

Morgantown, West Virginia

2013

Keywords: focal adhesion kinase (FAK), focal adhesions, nonmuscle cell, cell contraction, extracellular matrix, tension 


\section{ABSTRACT \\ The role of focal adhesion kinase in nonmuscle cell contraction}

\section{Kimberly M. Arnold}

Focal adhesions are specialized cell contact sites of distinct molecular composition and structure that bridge the actin cytoskeleton to the extracellular matrix and provide for efficient bidirectional transmission of biochemical and mechanical signals between the intra- and extracellular compartment. Many proteins within the focal adhesion have been discovered to be an integral component of the adhesion structure and function; however, a key molecule in the organization and physiological activity of focal adhesions is focal adhesion kinase (FAK). FAK is a nonreceptor tyrosine kinase that is essential for cell processes including cell migration, growth, and survival. Due to its connection between the cytoskeleton and extracellular matrix, FAK has been proposed to be a key component of integrin downstream signaling that regulates the organization of actin for transduction of cellular forces from inside to outside of the cell. While many studies have focused on determining FAK's role in sensing mechanical forces and regulating contractile signaling pathways, very few studies have attempted to determine the role of FAK in cell contraction through direct measurement of cellular tension. Therefore, the role of FAK in fibroblast and endothelial cell contractility was determined.

To investigate the role of FAK in endothelial cell tension and monolayer permeability, a stable FAK knockdown human pulmonary microvessel endothelial cell line (FAK-KD) was generated. Knockdown of FAK altered both cell morphology and actin distribution, and increased focal adhesion formation and VE-cadherin localization to cell-cell contacts. Measurement of tension produced by cells embedded within a three-dimensional (3-D) collagen matrix revealed that loss of FAK increased basal tension without alterations in basal myosin phosphorylation. Agonist-induced force was unaffected. However, loss of FAK enhanced endothelial monolayer barrier function. Thus, FAK is responsible for the balance between cellmatrix and cell-cell cohesion in order to regulate endothelial cell tension and monolayer permeability.

In order to determine the role of FAK in fibroblast cell contractility, FAK knockout (FAK-KO) mouse embryonic fibroblasts (MEFs) embedded in 3-D collagen gels were utilized. FAK null MEFs produced a decrease in basal tension and minimal agonist induced force compared to controls (FAK-WT). However, myosin II phosphorylation was comparable between FAK-KO and FAK-WT MEFs. Investigation of the collagen matrix revealed that FAKKO MEFs had an inability to organize their collagen matrix. Inhibition of FAK kinase activity or expression of FAK mutants revealed that FAK kinase activity was dispensable for tension generation. Thus, FAK localization to the focal adhesion was critical in the transmission of internal force to the collagen matrix resulting in cell contraction.

Collectively, these data show that FAK is an integral part in nonmuscle cellular tension. Although the loss of FAK altered tension generation differently in fibroblasts and endothelial cells, the differences in each cell's physiological function may explain why FAK regulates cell tension differently. Nevertheless, FAK is an important molecular player in focal adhesions facilitating the transduction of forces from inside to outside of the cell and may be a novel target in the development of treatments to control cell contractility. 


\section{Acknowledgements}

The past eight years have been the most challenging yet rewarding journey that I have ever had to face. I never would have made it through without the following people:

First and foremost, I would like to thank my mentor, Dr. Robert Wysolmerski, for his constant support and guidance throughout my graduate studies. He not only taught me critical scientific skills like experimental design and methods, but also to always ask questions and be curious in science. I thank him for his unending support, even when projects took an unexpected turn, and allowing me to grow as a scientist under his guidance.

I would also like to acknowledge the members of my dissertation committee, Dr. Jun Liu, Dr. Karen Martin, Dr. Fred Minnear, and Dr. Michael Schaller for their input and suggestions on my dissertation project and promoting my development as a scientist. To all of the faculty and students in the Biomedical Sciences Graduate Program at West Virginia University, particularly the Cellular \& Integrative Physiology program, I am grateful to have been a part of such a wonderful program and appreciate all the help and guidance they have given me.

Thank you to all my friends at WVU for their support and giving me the means to escape science when needed. I appreciate your friendship throughout the years. To my lab mate, Bridget, and my cubicle mates, Sarah and Mandy, I thank them for the laughs and needed Naticakes trips and especially a big thanks to Mandy for providing the "fuel" to write this dissertation. To my dear friends Kim, Steph, and Cristy, even though we were miles apart I thank them for always supporting me and listening when I needed someone to talk to. Finally, a special thank you to Becky for taking the time to provide feedback on part of this dissertation and always being there when I needed help, career advice, or just a good laugh.

To my parents, Rudy and Nancy, I cannot thank them enough for all their support they have given me throughout the years and promoting my curiosity in science. I know most of the time they did not fully understand what I was talking about or going through, but I thank them for listening and always being there when I needed them. Without their unwavering support in my educational pursuits I would have never been able to achieve my goals.

Finally, I would like to thank my husband, Raymond, for his unconditional love and support throughout my undergraduate and graduate studies. I can never thank him enough for staying in West Virginia with me after he graduated so I could finish my dissertation. Thank you for always being a great friend in good times and bad and providing a shoulder to cry on or an ear to listen. I appreciate that you always understood when I needed to spend a night working or a weekend doing experiments. Thank you for always believing in me and knowing that I could succeed. I could have not done this without you. 


\section{TABLE OF CONTENTS}

The role of focal adhesion kinase in nonmuscle cell contraction...........................................

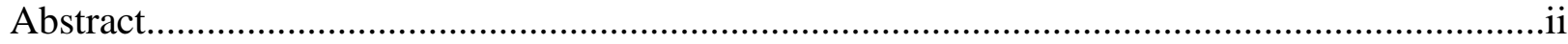

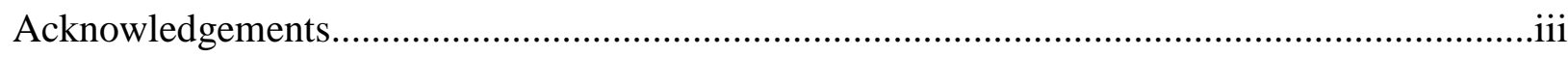

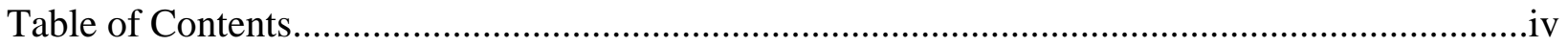

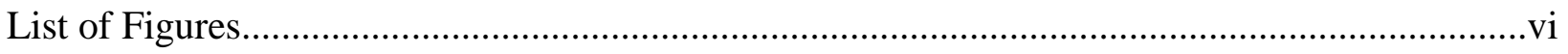

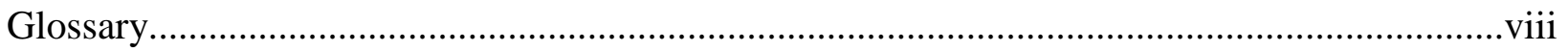

CHAPTER 1: Background and Significance............................................................

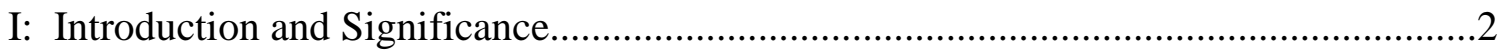

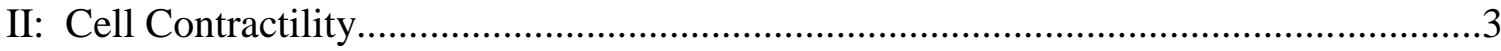

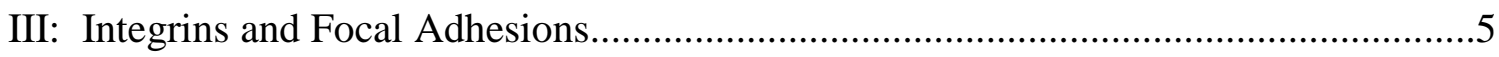

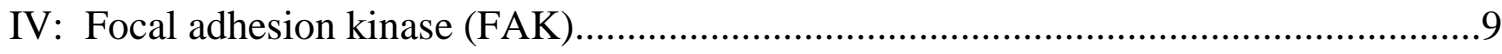

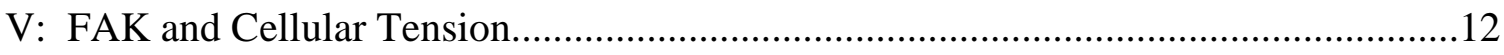

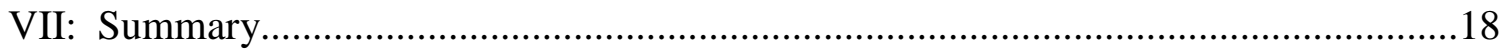

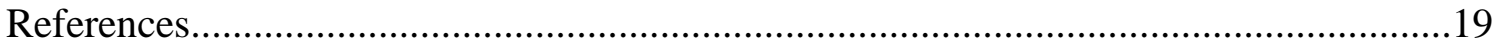

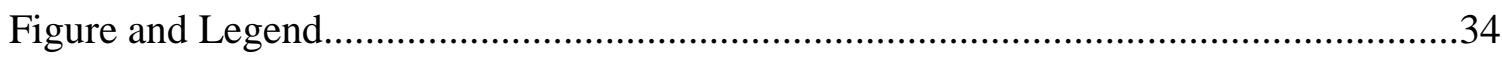

CHAPTER 2: Loss of focal adhesion kinase enhances endothelial barrier function and

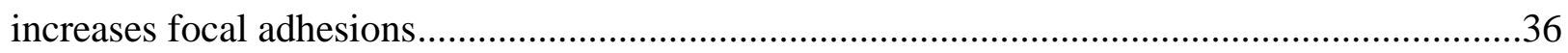

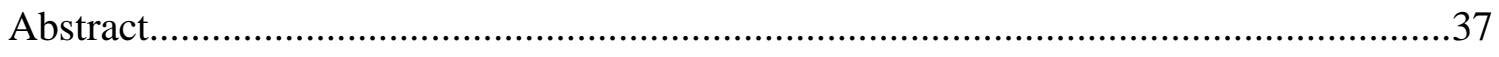

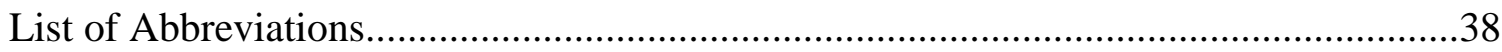

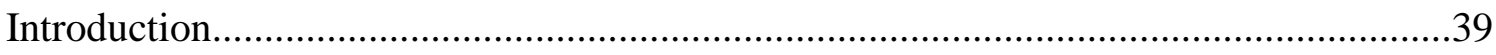

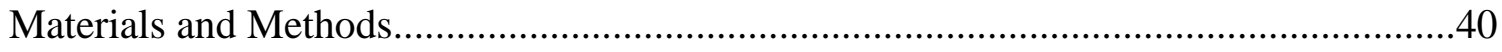

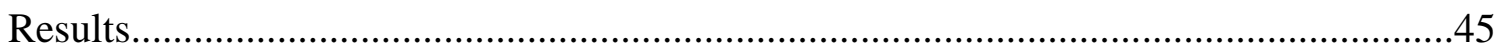




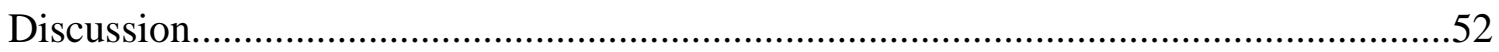

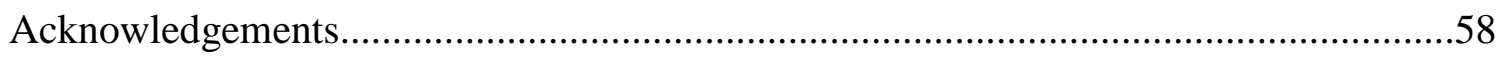

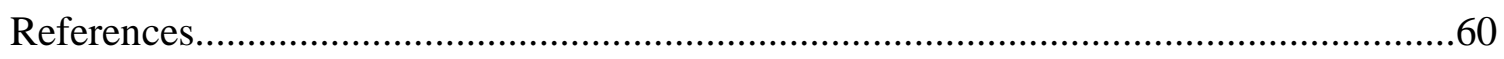

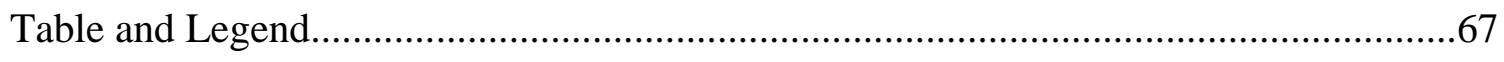

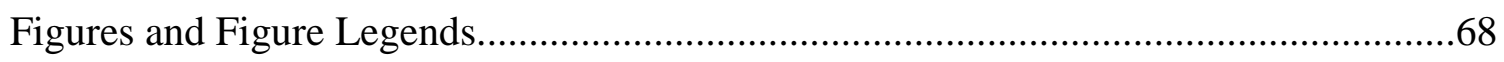

CHAPTER 3: Focal adhesion kinase (FAK) is necessary for tension generation and

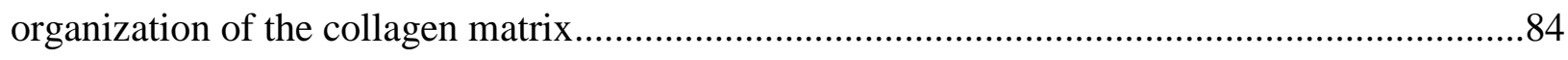

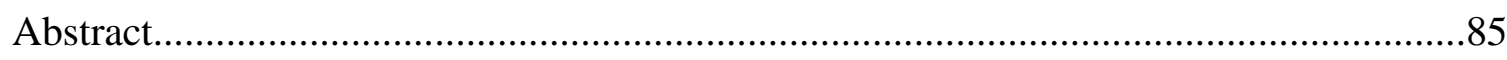

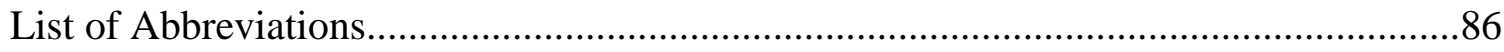

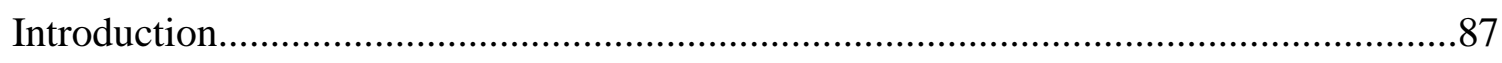

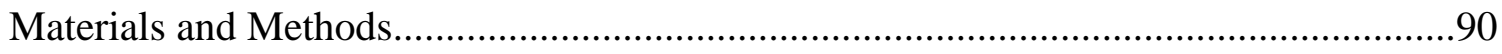

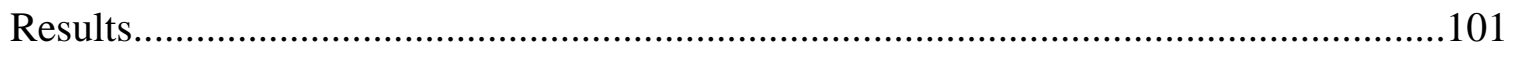

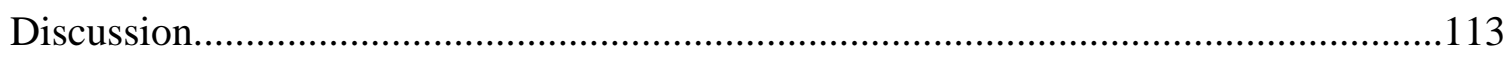

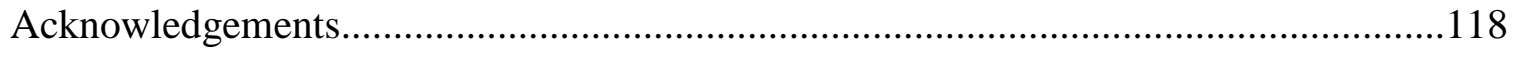

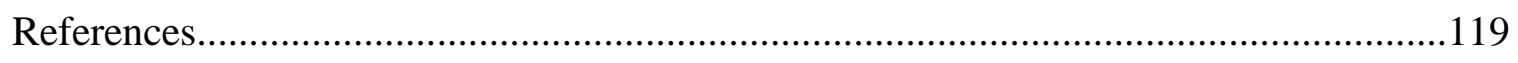

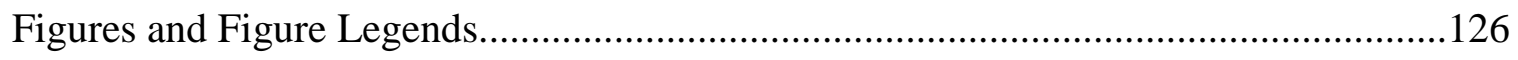

Supplementary Figures and Figure Legends.....................................................147

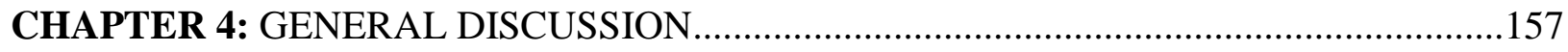

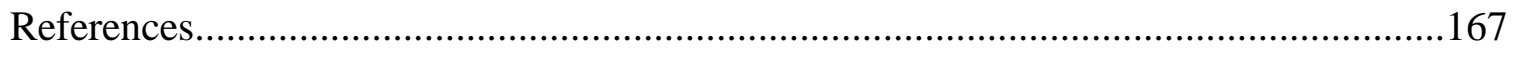

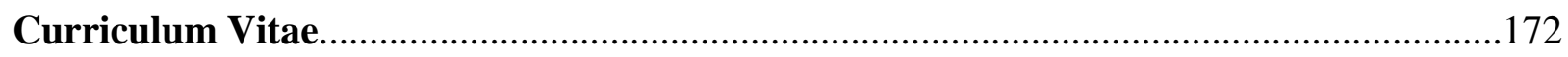




\section{LIST OF FIGURES}

\section{Chapter 1: Background and Significance}

1. Model of FAK's involvement in the regulation of cellular tension.

\section{Chapter 2: Loss of Focal Adhesion Kinase Enhances Endothelial Barrier Function and Increases Focal Adhesions}

Table 1: Effect of FAK knockdown on vinculin plaques..........................................67

1. FAK and Pyk2 protein expression in endothelial cells and MEFs............................68

2. Effect of FAK knockdown on cell proliferation and death......................................70

3. Effect of FAK knockdown on transendothelial electrical resistance..........................72

4. Localization of actin and vinculin in endothelial cells and MEFs..........................74

5. Vinculin protein expression in FAK-KD and WT endothelial cells...........................76

6. Effect of FAK knockdown on endothelial cell isometric tension and myosin II

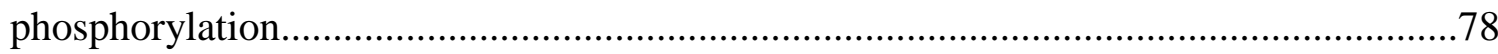

7. VE- and N-cadherin protein expression in FAK-KD and WT endothelial cells..........80

8. Localization of VE-cadherin and F-actin in WT and FAK-KD human pulmonary microvessel endothelial cells

Chapter 3: Focal adhesion kinase (FAK) is necessary for fibroblast tension generation and organization of collagen matrix in three-dimensional culture

1. Effect of FAK knockdown on basal and agonist induced tension generation and

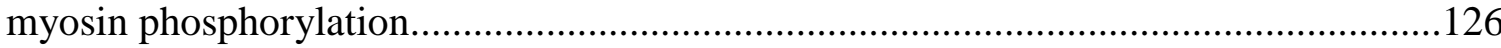

2. FAK knockout alters cell morphology in 2-D and 3-D....................................128

3. Effect of the loss of FAK on 3-D focal adhesion distribution and integrin protein

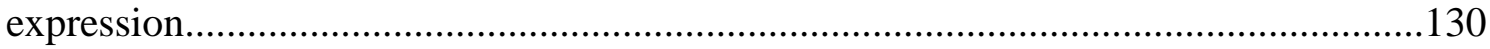

4. Loss of FAK alters MEF ability to compress 3-D collagen gel.............................132

5. Imaging of collagen matrix by second harmonic generation microscopy.................134

6. Effect of the loss of FAK on 3-D collagen matrix organization...............................136

7. Re-expression of FAK restores 3-D cell morphology, force generation and gel compression.

8. Re-expression of FAK restores collagen matrix organization 
9. FAK localization in focal adhesion and not kinase activity is important for tension

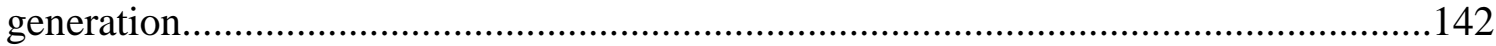

10. Mechanism of FAK regulation of fibroblast tension generation and collagen matrix organization

\section{Supplementary Figures}

1. Analysis of FAK, Pyk2, and p53 protein levels.................................................147

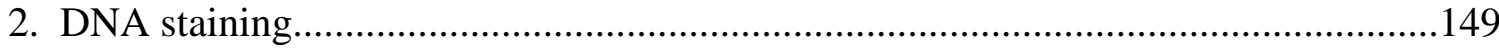

3. Re-expression of FAK restores cell spreading and focal adhesion formation

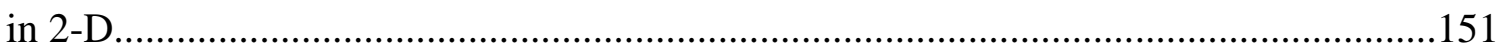

4. Restoration of SHG signal in FAK re-expression MEFs.....................................153

5. Inhibition of Src-family of kinases does not affect force generation........................155 


\section{GLOSSARY}

2-D - two-dimensional

3-D - three-dimensional

$\alpha$ - alpha chain of integrin heterodimer

ASAP1 - a phospholipid-dependent Arf GTPase-activating protein

$\beta$ - beta chain of integrin heterodimer

$\mathrm{BDM}$ - 2,3-butanedione monoxime

C4 - chloropyramine hydrochloride

Cdc42 - cell division control protein 42 homolog

CPI-17 - inhibitor of myosin phosphatase

CytoD - cytochalasian D

DOC - deoxycholate

ECIS - electric cell-substrate impedance sensing system

ECM - extracellular matrix

ERK - extracellular signal-regulated kinase

FAK - focal adhesion kinase

FAK180/3 - FAK knockout fibroblasts expressing constitutively active FAK

FAK-dead - FAK knockout fibroblasts expressing kinase dead mutant of FAK

FAK-KD - focal adhesion kinase knockdown human pulmonary microvessel endothelial cells

FAK-KO - focal adhesion kinase knockout mouse embryonic fibroblasts

FAK-WT - wild-type littermates of FAK-KO mouse embryonic fibroblasts

FAT - focal adhesion targeting domain of FAK

FERM - domain with sequence homology with band 4.1 and ezrin/radixin/moesin proteins

FRNK - FAK knockout fibroblasts expressing a truncated form of FAK

FRNK-C1034S - FAK knockout fibroblasts expressing FRNK targeting mutant

GAP - GTPase activating proteins

GDP - guanosine diphosphate

GEF - guanine nucleotide exchange factors

GRAF - a GTPase activating protein for Rho associated with FAK

GTP - guanosine triphosphate

HLEC - human pulmonary microvessel endothelial cells

ILK - integrin-linked kinase

$\mathrm{KO}$ - knockout

LARG - a Rho guanine nucleotide exchange factor

LPA - lysophosphatidic acid

MCP-1 - monocyte chemoattractant protein

MEFs - mouse embryonic fibroblasts

MLCK - myosin light chain kinase

MLCP - myosin light chain phosphatase 
MYPT1 - myosin phosphatase targeting subunit 1

p130Cas - p130 Crk-associated substrate

p190RhoGAP - a Rho GTPase activating protein

p190RhoGEF - a Rho guanine nucleotide exchange factor

p53-KO - p53 knockout mouse embryonic fibroblasts

PAGE - polyacrylamide gel electrophoresis

PAK - p21-activated kinase

PBS - phosphate buffered saline

PDZ-RhoGEF - a Rho guanine nucleotide exchange factor

Pyk2 - FAK-related proline-rich tyrosine kinase 2

Rac1 - Ras-related C3 botulinum toxin substrate 1

RGD - Arginine-Glycine-Aspartic Acid; amino acid sequence on integrin ligands

RhoA - Ras homolog gene family, member A

ROCK - Rho-associated kinase

RLC - nonmuscle myosin II regulatory light chain

SDS - sodium dodecyl sulfate

SEM - scanning electron microscopy

SHG - second harmonic generation

Src - nonreceptor tyrosine kinase

shRNA - short hairpin RNA

TBS - Tris-buffered saline

TER - transendothelial resistance

WT - wild-type control human pulmonary microvessel endothelial cells

WT-FAK - FAK knockout fibroblasts expressing full-length focal adhesion kinase 


\section{CHAPTER 1:}

\section{Background and Significance}




\section{I: Introduction and Significance}

Cell function requires the integration of molecular, biochemical, and biophysical signals and investigating how cells move, deform, and interact with each other and how they sense, generate, and respond to mechanical forces underlies the study of cell mechanics. The ability for a cell to sense and generate tension is pivotal for normal tissue morphology and function and is necessary in many physiological processes including embryogenesis, angiogenesis, and wound healing (Krieg et al., 2008; Lee et al., 2010; Wong et al., 2011). Understanding how a cell integrates mechanical forces within and transmits the tension to its external environment can aid in understanding how abnormal regulation of this process can contribute to the formation of disease. It has therefore been of recent interest to understand how cytoskeletal dynamics and cell adhesion with the underlying matrix contributes to the mechanical properties of the cell. Tension occurs in an object when there is a pulling force at one end and a resisting force at the other. In a cell, the pulling force is the contractile network of actin and myosin, and the resisting force is adhesion to other cells or with the cell's external surroundings. Cell adhesion to its underlying matrix anchors the cell allowing the generation of force, which aids in processes such as cell migration and growth. It is known that forces between cells and the cell with its external surroundings is critical for the generation of tension; however, how these structures are involved in the regulation of force development is not well understood. Also, the molecules that are critical in the transmission of this force to the underlying matrix have yet to be elucidated. Thus, this dissertation will focus on determining how the cell-matrix adhesion is involved in the generation and transmission of force by modulating expression of a key adhesion protein, focal adhesion kinase (FAK), in order to alter focal adhesion dynamics and measure changes in cellular tension. 


\section{II: Cell Contractility}

Cellular tension is regulated by myosin II activation and rearrangement of the actin cytoskeleton (Burridge and Chrzanowska-Wodnicka, 1996). Myosin II becomes activated upon phosphorylation of its regulatory light chains (RLC) allowing myosin bipolar filament assembly, interaction with actin cytoskeletal filaments, and stimulation of myosin II ATPase activity (Adelstein and Conti, 1975; Scholey et al., 1980; Tan et al., 1992). The phosphorylation status of myosin II can be regulated through direct phosphorylation of myosin II or inhibition of myosin light chain phosphatase (MLCP) (Alessi et al., 1992; Shimizu et al., 1994; Shirazi et al., 1994) through direct phosphorylation of myosin phosphatase targeting subunit 1 (MYPT1) (Trinkle-Mulcahy et al., 1995; Ichikawa et al., 1996; Ito et al., 2004) or indirectly via phosphorylation of CPI-17, which becomes a potent inhibitor of MLCP (Eto et al., 1995). Several kinases can catalyze the phosphorylation of myosin II including the calcium/calmodulindependent myosin light chain kinase (MLCK) (Scholey et al., 1980), p21-activated kinase (gamma-PAK) (Chew et al., 1998), intregrin-linked kinase (ILK) (Wilson et al., 2005), and Rhoassociated protein kinase (ROCK) (Amano et al., 1996a). Primary control of myosin II phosphorylation is through the serine/threonine kinase MLCK, which becomes activated following the binding of calcium dependent calmodulin resulting in direct phosphorylation of serine-19 and threonine-18 of myosin II RLC (Sellers, 1991; Tan et al., 1992). Serine-19 is the major site of phosphorylation with threonine-18 phosphorylation occurring at a slower rate.

Myosin II phosphorylation can also be regulated in a calcium-independent manner through the Ras homolog gene family, member A (RhoA). RhoA, a small GTPase protein and member of the Ras superfamily, is a key component in the regulation of the actin/myosin contractile machinery (Hall, 1994; Takai et al., 1995; Chrzanowska-Wodnicka and Burridge, 
1996). It is primarily involved in the bundling of stress fibers, which cluster integrins and promotes focal adhesion formation (Ridley and Hall, 1992; Chrzanowska-Wodnicka and Burridge, 1996). Control of RhoA activation is through GTP loading and unloading. The activation of RhoA through GTP loading is controlled by guanine nucleotide exchange factors (GEFs), which dissociate bound GDP from RhoA allowing the association of GTP. Conversely, inactivation of RhoA occurs through intrinsic hydrolysis of GTP by RhoA, which is initiated by GTPase-activating proteins (GAPs) (Hall, 1994; Burridge and Chrzanowska-Wodnicka, 1996). RhoA activation of its downstream effector ROCK, a serine/threonine kinase, can directly affect myosin II and actin cytoskeletal dynamics through two pathways (Amano et al., 1996a, 1996b; Clark et al., 1998; Nobes and Hall, 1999). ROCK can enhance myosin II activation through direct phosphorylation of myosin II RLC or by inactivation of MLCP, which increases myosin ATPase activity and promotes interaction between actin and myosin (Amano et al., 1996a; Kimura et al., 1996; Kureishi et al., 1997; Kawano et al., 1999; Totsukawa et al., 2000; Emmert et al., 2004). ROCK can also activate an actin-binding kinase known as LIM-kinase and alter cytoskeletal dynamics by regulating an actin severing protein called cofilin. When cofilin is in its active, dephosphorylated form, it can bind to F-actin and cause the severing of the actin filaments. When LIM-kinase becomes activated by ROCK, it specifically phosphorylates cofilin at serine 3, which affects cofilin's ability to bind F-actin, and thus inhibits the protein's ability to sever and depolymerize F-actin (Arber et al., 1998; Yang et al., 1998; Sumi et al., 1999). Phosphorylated cofilin may act to bundle and stabilize the actin filaments. Therefore, the actin/myosin contractile machinery, and thus, cell tension is altered by pathways that are regulating both myosin activity and cytoskeletal dynamics (Figure 1A). 


\section{III: Integrins and Focal Adhesions}

To begin to understand how the mechanical properties of cells are regulated, it is essential to determine what role key structures within the cell play in the regulation of this force. In order for the cell to generate tension, it needs to interact with its external environment. This interaction occurs when transmembrane receptors called integrins bind to extracellular matrix (ECM) proteins creating a physical linkage for the cell to its surroundings. Integrins are a large family of heterodimeric receptors that span the plasma membrane and bind to ECM proteins (e.g. fibronectin and collagen) via their large extracellular globular head domains (Ruoslahti, 1991; Hynes, 1992; van der Flier and Sonnenberg, 2001). The integrins bind to specific sequence motifs (Arginine-Glycine-Aspartic acid (RGD) sequence) present on ECM ligands (D’Souza et al., 1988). Integrins consist of an alpha $(\alpha)$ and a beta $(\beta)$ subunit. Most $\beta$ subunits can combine with multiple $\alpha$ subunits and a few of the $\alpha$ subunits can pair with multiple $\beta$ chains. Combinations of the $\alpha$ and $\beta$ chains yield a diverse family of receptors for ECM proteins (Hynes, 1992; Arnaout et al., 2005). Typically, the type of integrin found on a cell surface is dictated by the ECM proteins that the cell is adhered to (Dejana et al., 1988; Singer et al., 1988; Fath et al., 1989); however, it was found that a laminin-binding integrin $\alpha_{6} \beta_{1}$, typically not thought to bind to fibronectin, was detected on the cell surface of cells adhering to fibronectin (Cattelino et al., 1995). The specificity of the integrins to ECM proteins depends on differences in affinity of the integrin for the ECM proteins; hence, $\alpha_{5} \beta_{1}$ and $\alpha_{v} \beta_{3}$ can both bind fibronectin (Wu et al., 1993; Wu et al., 1996; Yang and Hynes, 1996). However, each integrin has been found to be essential in vivo even though the specificity of the integrins overlap in culture (Sheppard, 2000).

Internally, the integrins are linked to the contractile machinery (actin and myosin) through multiprotein complexes called focal adhesions, which consist of adaptor proteins that 
bind to the integrin's short cytoplasmic tails (Burridge et al., 1988; Jockusch et al., 1995; Liu et al., 2000). Focal adhesions are flat, elongated structures located around the periphery of the cell at the ends of actin stress fibers (Abercrombie and Dunn, 1975; Izzard and Lochner, 1976; Sastry and Burridge, 2000; Zamir and Geiger, 2001). Typically, focal adhesions are thought to be an anomaly of cells grown on a solid surface; however, there is evidence of these structures in vivo (e.g. aortic endothelial cells form adhesions with the underlying basement membrane (Kano et al., 1996), myotendinous junctions are formed between skeletal muscles cells and tendon collagen fibers (Tidball, 1983, Tidball, 1984; Tidball and Daniel, 1986; Trotter et al. 1981, Trotter et al., 1983), and membrane bound dense plaques were observed in smooth muscle cells (Turner et al., 1991)).

The cytoplasmic domain of $\beta$-integrins is critical for focal adhesion formation and targeting the integrin to the plasma membrane (Solowska et al., 1989; Hayashi et al., 1990; Marcantonio et al., 1990; Geiger et al., 1992; LaFlamme et al., 1992). Following binding to the ECM, a conformational change is induced in the cytoplasmic domain of the integrins, unmasking the short cytoplasmic tails, and allowing the interaction with focal adhesion proteins (Hynes, 2002; Puklin-Faucher and Sheetz, 2009). Previous studies have shown that cells seeded on substrates covalently linked with a low density of short synthetic peptides containing the RGD sequence that integrins bind to does not induce cell spreading or formation of focal adhesions. Increasing the density of the peptides but keeping significant space between them results in cell spreading without focal adhesion formation. It is not until the peptides are at high densities and close together that the cells are able to form focal adhesions indicating that integrins need to be clustered in order for focal adhesions to form (Massia and Hubbell, 1991). Integrins have no intrinsic enzymatic activity, and therefore, clustering integrins following ligand occupancy 
triggers the recruitment of key kinases and focal adhesion proteins such as talin, vinculin, $\alpha$ actinin, paxillin, and FAK to elicit downstream signaling cascades (Miyamoto et al., 1995a, 1995b). Upon binding to the ECM, integrin clustering results in an increase in protein tyrosine phosphorylation in a variety of cell types including fibroblasts, carcinoma cells, $\mathrm{T}$ lymphocytes, B cells, platelets, and neutrophils (Golden et al., 1990; Guan et al., 1991; Kornberg et al., 1991; Nojima et al., 1992; Freedman et al., 1993; Kanner et al., 1993) allowing external signals to alter cell behavior. Internally, the focal adhesions are linked to the cellular cytoskeleton through a variety of actin binding proteins although the hierarchal structure of the adhesion is complex and may vary depending on the condition (Jockusch et al., 1995; Hynes, 2002; Zaidel-Bar and Geiger, 2010). Currently, there are roughly 180 documented components of focal adhesions with 742 direct interactions between these components with this number continuing to grow, indicating the complexity in the network of proteins that form the adhesion (Zaidel-Bar and Geiger, 2010). Nevertheless, focal adhesions create a bridge between the actin cytoskeleton and the ECM allowing for bidirectional signaling where the cell can respond to external cues (outside-in signaling) as well as influence its own surroundings (inside-out signaling) (Hynes, 2002).

A cell can sense changes in external or internal tension and respond either through alteration of adhesive sites or through activation of signaling pathways to alter cell behavior. The ability for the cell to accomplish this is known as mechanotransduction. Focal adhesions are a prime candidate in sensing changes in tension due to their close proximity to the plasma membrane, connections with the ECM and actin cytoskeleton, and abundance of signaling proteins within the complex. Mechanical stimulation results in modulation of cell spreading, remodeling of the actin cytoskeleton, activation of actin/myosin interactions, recruitment of 
integrins, and reinforcement of focal adhesions and cytoskeletal structures (Schwartz, 2010). Focal adhesion formation will increase in response to external forces being applied to the cell and disassemble when force is removed (Balaban et al., 2001; Riveline et al., 2001). Mechanosensitive focal adhesions underlie a number of behaviors including development and maintenance of bone, blood vessels and myocardium, regulation of blood pressure, and myometrial remodeling (Cattaruzza et al., 2004; Danowski et al., 1992; Sharp et al., 1997; Simpson et al., 1993; Wozniak et al., 2000; Wu et al., 2008).

Focal adhesions are also able to sense the tension that the cell generates and transmit force through the cellular membrane to the ECM leading to remodeling of the ECM fibers and establishment of a denser, highly cross-linked matrix (Larsen et al., 2006). Cells will exert stronger forces on a matrix that is more rigid, and therefore, the organization of the matrix is an indication of internal cellular tension. Rigidity of the matrix also plays a role in focal adhesion formation. Cells adhering to rigid surfaces form more robust adhesions compared to those seeded onto soft, pliable substrates (Choquet et al., 1997; Guo et al., 2006) most likely due to the rigid surfaces' capability of resisting strong contractile forces of the cell. Treatment of cells with lysophosphatidic acid (LPA), thrombin, or bombesin increases Rho activity and subsequently increases contractile activity of the cell and focal adhesion size (Ridley and Hall, 1992; Chrzanowska-Wodnicka and Burridge, 1996; Ishida et al., 1999; Totsukawa et al., 2000). Inhibition of cell tension causes focal adhesions to disassemble (Chrzanowska-Wodnicka and Burridge, 1996). The ability for focal adhesions to respond to changes in internal and external forces is an inherent property of adhesions and is important for cell function such as cell migration and growth. Many of the focal adhesion proteins have been under investigation to determine their involvement in responding to external forces and in the generation and 
transmission of force; however a key protein that has become a prime candidate for sensing and generating forces is focal adhesion kinase (FAK).

\section{IV: Focal adhesion kinase (FAK) structure and function}

FAK is a ubiquitously expressed nonreceptor tyrosine kinase named after its subcellular localization (Kanner et al., 1990; Schaller et al., 1992, Kornberg et al., 1991; Guan et al., 1991; Hanks et al., 1992). It is a key structural and functional protein involved in signaling pathways known to control diverse cellular processes including cell adhesion, migration, polarity, growth, and survival (Ilic et al., 1995; Ilic et al., 1998; Zhao et al., 1998; Owen et al., 1999; Renshaw et al., 1999; Sieg et al., 1999; Sieg et al., 2000; Webb et al., 2002). FAK activity is also critical in normal tissue function and disease including vascular development (Shen et al., 2005; Braren et al., 2006), dendrite formation, axon outgrowth (Watanabe et al., 2008), skin fibrosis (Wong et al., 2011), cardiomyocyte-induced hypertrophy (Peng et al., 2008), and lung fibrosis (Lagares et al., 2012). FAK protein levels are elevated in benign, preinvasive, and invasive tumors (Gabarra-Niecko et al., 2003) and dominant-negative inhibition of FAK reduces in vivo primary mammary tumor growth and lung metastasis formation (van Nimwegen et al., 2005).

The protein structure of FAK suggests that it acts as both an adaptor protein and as a kinase. FAK protein consists of three main domains: a central catalytic domain flanked on the N-terminus by a region of sequence homology with band 4.1 and ezrin/radixin/moesin (ERM) proteins termed the FERM domain (Frame et al., 2010) and on the C-terminus by a focal adhesion targeting (FAT) domain (Hildebrand et al., 1993). The FERM domain serves as a site of interaction with the cytoplasmic domain of $\beta$-integrins, ezrin, and growth factor receptors (Schaller et al., 1995; Chen et al., 2000; Sieg et al., 2000; Poullet et al., 2001). The FAT domain 
is necessary for localization of FAK to the focal adhesions as well as promoting the binding of proteins such as paxillin and p190RhoGEF (Hildebrand et al., 1993; Hildebrand et al., 1995; Tachibana et al., 1995; Cooley et al., 2000; Zhai et al., 2003). Also, in between the FAT and the kinase domain, there is a proline-rich region providing binding sites for SH3-domain containing proteins which includes p130Cas (p130 Crk-associated substrate), GRAF (a $\underline{\text { GAP for }}$ Rho associated with focal adhesion kinase), and ASAP1 (Harte et al., 1996, Hildebrand et al., 1996; Taylor et al., 1998, Liu et al., 2002).

Under normal conditions, FAK is inactivated by autoinhibition of the kinase domain through binding of the FERM domain (Cooper et al., 2003; Jacamo and Rozengurt, 2005; Lietha et al., 2007). Upon integrin engagement with the ECM, FAK is recruited to focal adhesions and dimerizes at the cytoplasmic face of the membrane. This relieves the authoinhibition and initiates phosphorylation of tyrosine-397 of FAK, thus activating kinase activity (Kornberg et al., 1992; Katz et al., 2002; Cooper et al., 2003). Phosphorylation of FAK provides a binding site for Src, locking both FAK and Src in an activated state, generating binding sites for signaling proteins with $\mathrm{SH} 2$ domains, and creating a kinase complex that results in the phosphorylation and activation of downstream proteins including paxillin and p130Cas (Schaller et al., 1994; Bellis et al., 1995; Miyamoto et al 1995b; Polte and Hanks, 1995; Schaller and Parsons, 1995; Cary et al., 1998; Thomas et al., 1999). Following activation of these proteins, other molecules are then recruited to the adhesions and regulate the organization of the actin cytoskeleton (Parsons, 1993; Schaller and Parsons, 1994; Richardson and Parsons, 1995). Mutation of the FAT domain impairs FAK autophosphorylation and localization to focal adhesions, resulting in the inability to phosphorylate FAK substrates in response to cellular adhesion (Shen and 
Schaller, 1999). Therefore, FAK is an important component in the transmission of extracellular signals into the cell through activation of several cellular signaling pathways.

The molecular biology and biochemistry of FAK has been well documented, however, FAK's physiological role is still being uncovered. Knockout (KO) of FAK in mice is lethal at embryonic day 8.5 due to severe defects in mesoderm and cardiovascular development as a result of impaired cell migration (Furuta et al., 1995; Ilic et al., 1995). Vascular defects in FAK KO mice result from the inability of endothelial cells to organize themselves into a vascular network and not a defect in tissue-specific differentiation (Ilic et al., 2003). An endothelial specific KO of FAK during late embryogenesis is also lethal in mice due to defective vasculogenesis resulting in impaired vasculature and associated hemorrhaging and edema formation, indicating FAK plays a role in angiogenesis and vascular development (Shen et al., 2005). Fibroblasts derived from FAK KO mice embryos form enlarged focal adhesions, have a decrease in cell migration, and have a rounded, contractile cell morphology due to a decrease in cell spreading, implying that there is a lack of focal adhesion disassembly or "turnover" (Ilic et al., 1995). An increase in stable adhesions and tensile stress fibers is thought to lead to an increase in cellular tension which will alter the cell shape and reduce the cell's ability to migrate. Keratinocytes derived from mice with an epidermal-specific KO of FAK appear more contractile with large adhesions and an abundance of tensile stress fibers (Schober at al., 2007). However, FAK knockdown in fibroblasts, epithelial, or endothelial cells, or fibroblast and neurons derived from mice with a conditional $\mathrm{KO}$ of FAK have an elongated or spindle-shaped morphology consistent with the inability of the cells to contract (Rico et al., 2004; Yano et al., 2004; Tilghman et al., 2005; Braren et al., 2006). Also, FAK deficient endothelial and HeLa cells show an increase in cell motility compared to the decrease in motility observed by FAK-null fibroblasts (Yano et al., 
2004; Tilghman et al., 2005; Braren et al., 2006). Thus, there is a potential role for FAK in the regulation of cellular tension.

\section{V: FAK and cell tension}

\section{FAK and mechanotransduction}

Modulation of FAK expression results in changes in cell morphology and adhesion size implicating an alteration in cellular contractile activity; however, it is only suggestive of FAK's involvement in the regulation of cellular tension. One well documented role for FAK in tension generation is through its ability to act as a mechanotransducer. In mechanically stimulated fibroblasts, FAK is the main protein found to be involved in the response to the stimuli (Wang et al., 2001; Sawada and Sheetz, 2002; Torsoni et al., 2003). Wong and colleagues (2011) have also found that physical force can enhance skin fibrosis through the activation of FAK which stimulates ERK (extracellular signal-regulated kinase) activity and the secretion of monocyte chemoattractant protein-1 (MCP-1). Furthermore, studies have shown that extracellular force or stress can activate FAK and result in an increase in tension (Li et al., 1997; Sawada and Sheetz, 2002). Increases in cellular contractility by treatment with LPA or bombesin increases focal adhesion formation and FAK activation possibly due to FAK recruitment to focal adhesions or physical stretching of the FAK molecule to remove the autoinhibition of the kinase activity (Sinnett-Smith et al., 1993; Seufferlein and Rozengurt, 1994). Similarly, inhibition of myosin activity through blebbistatin treatment or Rho activity through the presence of a ROCK inhibitior decreased focal adhesion size and FAK activity in fibroblasts (Pasapera et al., 2010). FAK signaling has also been found to be required in load-induced cardiac hypertrophy and bone remodeling in response to mechanical loading (Clemente et al., 2007; Leucht et al., 2007) 
indicating that FAK is responsible for sensing external forces and integrating them internally to alter focal adhesion dynamics and actinomyosin contractility.

Mechanical forces inside of the cell can be modulated by the rigidity of the ECM. Cells can sense the rigidity of the matrix that they are seeded on or in and adjust the tension that they exert (Choquet et al., 1997; Lo et al., 2000; Saez et al., 2005). Studies have shown that FAK may be critical in this process. Fibroblasts normally migrate towards a stiffer substrate (i.e. higher substrate rigidity); however, FAK KO fibroblasts do not show a preference for stiffness (Lo et al., 2000; Wang et al., 2001). Hence, FAK is responsible in sensing the rigidity of the substrate and therefore changes in tension. FAK can also be involved in the strengthening of the adhesions in response to changes in tension. Control fibroblasts have an increase in traction forces when substrate rigidity increases, which coincides with the formation of actin stress fibers and robust focal adhesions; however, FAK KO fibroblasts maintain lower traction forces (Wang et al., 2001). Correlation between FAK activity and adhesion growth, as well as increases in focal adhesion strength in response to changing forces, suggests that FAK may be involved in a positive feedback loop involving focal adhesion growth, FAK activation, and tension generation.

\section{FAK and contractile signaling pathways}

Numerous studies have shown the involvement of FAK in signaling pathways that regulate cellular contractile activity. Integrin engagement recruits FAK to the focal adhesion along with other scaffolding and signaling proteins which associate with additional molecules to regulate signaling to Rho-GTPases. Integrin signaling networks regulate the activation of the family of Rho-GTPases (Rho, Rac1, Cdc42) through recruitment of associated GAPs and GEFs to focal adhesions. Rho in particular regulates adhesion assembly and disassembly by activating 
pathways that lead to cell contraction and actin polymerization (Figure 1A). Studies have shown that FAK is not needed for focal adhesion assembly but rather necessary for disassembly (Ren et al., 2000; Giannone et al., 2004; Webb et al., 2004; Ezratty et al., 2005). In migrating cells, FAK can cause a local decrease in cell tension to disassemble adhesions by acting on signaling pathways which include ERK and MLCK (Webb et al., 2004). Loss of FAK in keratinocytes has shown to cause a decrease in active Src and p190RhoGAP, inactivation of MLCP, and a slight increase in myosin II phosphorylation (Schober et al., 2007). Thus, FAK can downregulate myosin activation by altering MLCK and Rho activity.

It is known that upon adhesion to the ECM, there is a transient decrease in RhoA activity followed by extended RhoA activation that eventually decreases over time (Ren et al., 1999). However, fibroblasts devoid of FAK are unable to downregulate RhoA following adhesion and levels of active RhoA are sustained for a period of several hours (Ren et al., 2000; Pirone et al., 2006). FAK has been found to be able to suppress RhoA activity via p190RhoGAP phosphorylation (Holinstat et al., 2006). Recent indirect evidence through inhibitor studies has shown that the loss of FAK in fibroblasts also results in an increase in ROCK and MLCK activity (Chen et al., 2002). Inhibition of RhoA or ROCK in FAK null fibroblasts decreases focal adhesion formation and restores cell spreading (Ren et al., 2000; Chen et al., 2002). Inhibition of myosin ATPase activity by treatment with 2,3-butanedione monoxime (BDM) in FAK KO fibroblasts allowed cell spreading suggesting that increased myosin-driven contractility in FAK KO fibroblasts is causing a decrease in cell spreading. However, treated cells did not form tensile stress fibers (Chen et al., 2002).

Thrombin is a potent agonist of cell contractility and binding to its receptor leads to an increase in Rho activation and myosin II phosphorylation (Giuliano and Taylor, 1990; Goeckeler 
and Wysolmerski, 1995; Vouret-Craviari et al., 1998). Rho can be activated by multiple GEFs downstream of FAK signaling. Treatment with thrombin activates FAK in both a Rhodependent and -independent mechanism and PDZ-RhoGEF and LARG (a Rho GEF) can in turn be activated by FAK enhancing the activation of Rho in vivo (Chikumi et al., 2002). Expression of a transmembrane-anchored chimeric form of FAK results in constitutive FAK kinase activity (Chan et al., 1994) and increases in RhoA activation, which is mediated by PDZ-RhoGEF. Blockade of FAK activity through expression of a noncatalytic carboxyl-terminal domain of FAK called FRNK (ㅌK-related non-tyrosine kinase) (Schaller et al., 1993; Richardson and Parsons, 1996; Nolan et al., 1999) inhibited the phosphorylation of PDZ-RhoGEF and thus RhoA activation (Chikumi et al., 2002). In neurons, the p190RhoGEF has been shown to interact with the FAT domain of FAK resulting in phosphorylation of GEF and activation of RhoA following adhesion (Zhai et al., 2003). FAK is also important for activation of Rho-GTPases family member, Rac1, which is involved in lamellipodium formation via the assembly of the cortical actin network (Burridge and Wennerberg, 2004). Activation of the Rac pathway by FAK may provide signals promoting protrusiveness in areas of the cell under tension and this coupling is critical for the maintenance of polarity during cell migration.

FAK has also been linked to the downregulation of Rho through the Rho GAPs which enhance the intrinsic GTPase activity of Rho-GTPases, rendering them inactive. The Rho GAP, GRAF, localizes to stress fibers and focal adhesions and binds to the proline rich region of FAK (Taylor et al., 1998; Taylor et al., 1999). p190RhoGAP is activated by Src at focal adhesions (Arthur et al., 2000) and in endothelial cells, FAK associates and phosphorylates it upon thrombin stimulation (Holinstat et al., 2006). p190RhoGAP does not localize to focal adhesions in skin keratinocytes in which FAK expression was conditionally ablated resulting in elevated 
Rho activity and enhanced stress fibers (Schober et al., 2007). Abnormal regulation of Rho and the enzymes that activate myosin II and regulate cytoskeletal dynamics implicates a role for FAK in the regulation of cellular tension generation.

Based on current literature, it is hypothesized that if FAK is knocked out of the cell, there are alterations in focal adhesion dynamics and an elevation of Rho through modulation of RhoGAPs and RhoGEFs. Active Rho can activate ROCK, causing an increase in myosin activity through direct phosphorylation of myosin II RLC or indirectly through inhibition of MLCP. ROCK can enhance the stability of the cytoskeleton by enhancing actin polymerization and stress fiber formation. The combination of enhanced myosin activity and cytoskeletal stability is thought to lead to an overall increase in cellular tension (Figure 1B). Therefore, it is hypothesized that a loss of FAK results in an overall increase in cellular tension.

\section{FAK and measurements of force generation}

Observing changes in contractile signaling pathways downstream of FAK do not clarify FAK's role in cellular tension generation. Although the role of FAK as a mechanosensor has been well documented, FAK's role in the generation and transmission of adhesive forces is limited. Magnetic tweezers exert mechanical shear stress to the cell by applying forces to substrate coated superparamagnetic beads and reports measurement of cell stiffness (Kollmannsberger and Fabry, 2007). Atomic force microscopy has also been used as an alternative method to measure cell stiffness. Cell internal forces that resist cell shape changes can be estimated from cell stiffness measurements. Since FAK promotes high focal adhesion turnover and cell motility, it would be expected that loss of FAK would result in a less dynamic cytoskeleton and more a rigid cell. However, FAK KO cells are rounded and have an increase in 
cortical actin and loss of stress fibers which are signs of reduced cell stiffness. FAK KO fibroblasts have reduced cell stiffness as measured by magnetic tweezers and atomic force microscopy and an unstable, dynamic cytoskeleton as measured by nanoscale particle tracking (Klemm et al., 2009; Fabry et al., 2011). Therefore, FAK is important in maintaining cell rigidity through promoting a static and highly aligned cytoskeleton (Fabry et al., 2011)

A limited number of studies have focused on directly measuring the tension transmitted across the adhesion onto the underlying substrate and the role FAK plays in this transmission. Through use of an adhesion strength assay using micropatterned substrates (Gallant et al., 2002), it is shown that FAK regulates the time-dependent generation of adhesive force following integrin activation (Michael et al., 2009). In addition, utilization of a microfabricated force sensor consisting of an array of vertically placed microneedles which report the traction force exerted by the cells to the underlying substrate (Tan et al., 2003) reveals that expression of FRNK increased traction forces where as expression of an autophosphorylation-defective mutant of FAK decreases traction force indicating that FAK alters the cytoskeletal tension and forces experienced at the adhesion (Pirone et al., 2006).

Previous studies show that FAK is a necessary component of the signaling pathways that regulate smooth muscle contraction. Upon stimulation of smooth muscle cells, FAK is activated by tyrosine phosphorylation (Tang et al., 1999). When FAK is knocked down in these cells, there is a decrease in stimulated force production accompanied by a decrease in intracellular free calcium and myosin activation (Tang and Gunst, 2001). It appears that FAK both responds to and causes changes in mechanical force linking cell adhesion to changes in cell mechanics. These studies point to a critical role for FAK in the regulation of smooth muscle and nonmuscle cellular tension production; however, these studies are only suggestive of a role for FAK in the 
generation and transmission of cellular tension. There is also no clear evidence linking FAK to actual changes in overall cell contractility, particularly in nonmuscle cells. In addition, a majority of these studies take a reductionist approach and look at singe cells or cells cultured on a substrate in a dish. Physiologically, cells behave in a cohesive unit. Therefore, studying cellular contractility in a three-dimensional (3-D) system would be more indicative of cell behavior in vivo.

\section{VII: Summary}

To determine how the interaction between the ECM, adhesion complexes, and cellular contractile machinery generates force and to identify the signaling pathways that are involved would be a considerable task. Therefore, this dissertation focuses on assessing how focal adhesions are involved in the generation of force production in nonmuscle cells. In order to address focal adhesion involvement in tension generation, expression of FAK in fibroblasts and endothelial cells was modulated. The overall hypothesis of this dissertation is that FAK is critical for force generation in nonmuscle cells. In order to investigate FAK's role in cellular tension, a 3-D culture system was utilized to measure changes in cell contractility. The second chapter of this dissertation investigates the effect of FAK knockdown in human pulmonary microvessel endothelial cells on force generation and endothelial barrier function. The third chapter of this dissertation utilizes FAK-KO MEFs to look at how $\mathrm{KO}$ of FAK alters force generation and how this effects collagen matrix organization in a 3-D environment. Also, the role of FAK kinase activity in force generation of fibroblasts was determined. 


\section{References}

Abercrombie, M., and G.A. Dunn. 1975. Adhesions of fibroblasts to substratum during contact inhibition observed by interference reflection microscopy. Exp. Cell Res. 92:57-62.

Adelstein, R.S., and M.A. Conti. 1975. Phosphorylation of platelet myosin increases actinactivated myosin ATPase activity. Nature. 256:597-598.

Alessi, D., L.K. MacDougall, M.M. Sola, M. Ikebe, and P. Cohen. 1992. The control of protein phosphatase-1 by targetting subunits. The major myosin phosphatase in avian smooth muscle is a novel form of protein phosphatase-1. Eur. J. Biochem. 210:1023-1035.

Amano, M., M. Ito, K. Kimura, Y. Fukata, K. Chihara, T. Nakano, Y. Matsuura, and K. Kaibuchi. 1996a. Phosphorylation and activation of myosin by Rho-associated kinase (Rhokinase). J. Biol. Chem. 271:20246-20249.

Amano, M., H. Mukai, Y. Ono, K. Chihara, T. Matsui, Y. Hamajima, K. Okawa, A. Iwamatsu, and K. Kaibuchi. 1996b. Identification of a putative target for Rho as the serine-threonine kinase protein kinase N. Science. 271:648-650.

Arber, S., F.A. Barbayannis, H. Hanser, C. Schneider, C.A. Stanyon, O. Bernard, and P. Caroni. 1998. Regulation of actin dynamics through phosphorylation of cofilin by LIM-kinase. Nature. 393:805-809.

Arnaout, M.A., B. Mahalingam, and J.P. Xiong. 2005. Integrin structure, allostery, and bidirectional signaling. Annu. Rev. Cell Dev. Biol. 21:381-410.

Arthur, W.T., L.A. Petch, and K. Burridge. 2000. Integrin engagement suppresses RhoA activity via a c-Src-dependent mechanism. Curr. Biol. 10:719-722.

Balaban, N.Q., U.S. Schwarz, D. Riveline, P. Goichberg, G. Tzur, I. Sabanay, D. Mahalu, S. Safran, A. Bershadsky, L. Addadi, and B. Geiger. 2001. Force and focal adhesion assembly: a close relationship studied using elastic micropatterned substrates. Nat. Cell Biol. 3:466-472.

Bellis, S.L., J.T. Miller, and C.E. Turner. 1995. Characterization of tyrosine phosphorylation of paxillin in vitro by focal adhesion kinase. J. Biol. Chem. 270:17437-17441.

Braren, R., H. Hu, Y.H. Kim, H.E. Beggs, L.F. Reichardt, and R. Wang. 2006. Endothelial FAK is essential for vascular network stability, cell survival, and lamellipodial formation. $J$. Cell Biol. 172:151-162.

Burridge, K., and M. Chrzanowska-Wodnicka. 1996. Focal adhesions, contractility, and signaling. Annu. Rev. Cell Dev. Biol. 12:463-519.

Burridge, K., K. Fath, T. Kelly, G. Nuckolls, and C. Turner. 1988. Focal adhesions: transmembrane junctions between the extracellular matrix and the cytoskeleton. Annu. Rev. Cell Biol. 4:487-525. 
Burridge, K., and K. Wennerberg. 2004. Rho and Rac take center stage. Cell. 116:167-179.

Cary, L.A., D.C. Han, T.R. Polte, S.K. Hanks, and J.L. Guan. 1998. Identification of p130Cas as a mediator of focal adhesion kinase-promoted cell migration. J. Cell Biol. 140:211-221.

Cattaruzza, M., C. Lattrich, and M. Hecker. 2004. Focal adhesion protein zyxin is a mechanosensitive modulator of gene expression in vascular smooth muscle cells. Hypertension. 43:726-730.

Cattelino, A., R. Longhi, and I. de Curtis. 1995. Differential distribution of two cytoplasmic variants of the alpha 6 beta 1 integrin laminin receptor in the ventral plasma membrane of embryonic fibroblasts. J. Cell Sci. 108:3067-3078.

Chan, P.Y., S.B. Kanner, G. Whitney, and A. Aruffo. 1994. A transmembrane-anchored chimeric focal adhesion kinase is constitutively activated and phosphorylated at tyrosine residues identical to pp125FAK. J. Biol. Chem. 269:20567-20574.

Chew, T.L., R.A. Masaracchia, Z.M. Goeckeler, and R.B. Wysolmerski. 1998. Phosphorylation of non-muscle myosin II regulatory light chain by p21-activated kinase (gamma-PAK). $J$. Muscle Res. Cell Motil. 19:839-854.

Chen, B.H., J.T. Tzen, A.R. Bresnick, and H.C. Chen. 2002. Roles of Rho-associated kinase and myosin light chain kinase in morphological and migratory defects of focal adhesion kinasenull cells. J. Biol. Chem. 277:33857-33863.

Chen, L.M., D. Bailey, and C. Fernandez-Valle. 2000. Association of beta 1 integrin with focal adhesion kinase and paxillin in differentiating Schwann cells. J. Neurosci. 20:3776-3784.

Chikumi, H., S. Fukuhara, and J.S. Gutkind. 2002. Regulation of G protein-linked guanine nucleotide exchange factors for Rho, PDZ-RhoGEF, and LARG by tyrosine phosphorylation: evidence of a role for focal adhesion kinase. J. Biol. Chem. 277:12463-12473.

Choquet, D., D.P. Felsenfeld, and M.P. Sheetz. 1997. Extracellular matrix rigidity causes strengthening of integrin-cytoskeleton linkages. Cell. 88:39-48.

Chrzanowska-Wodnicka, M., and K. Burridge. 1996. Rho-stimulated contractility drives the formation of stress fibers and focal adhesions. J. Cell Biol. 133:1403-1415.

Clark, E.A., W.G. King, J.S. Brugge, M. Symons, and R.O. Hynes. 1998. Integrin-mediated signals regulated by members of the Rho family of GTPases. J. Cell Biol. 142:473-586.

Clemente, C.F., T.F. Tornatore, T.H. Theizen, A.C. Deckmann, T.C. Pereira, I. Lopes-Cendes, J.R. Souza, and K.G. Franchini. 2007. Targeting focal adhesion kinase with small interfering RNA prevents and reverses load-induced cardiac hypertrophy in mice. Circ Res. 101:13391348. 
Cooley, M.A., J.M. Broome, C. Ohngemach, L.H. Romer, and M.D. Schaller. 2000. Paxillin binding is not the sole determinant of focal adhesion localization or dominant-negative activity of focal adhesion kinase/focal adhesion kinase-related nonkinase. Mol. Biol. Cell. 11:32473263.

Cooper, L.A., T.L. Shen, and J.L. Guan. 2003. Regulation of focal adhesion kinase by its amino-terminal domain through an autoinhibitory interaction. Mol. Cell. Biol. 23:8030-8041.

Danowski, B.A., K. Imanaka-Yoshida, J.M. Sanger, and J.W. Sanger. 1992. Costameres are sites of force transmission to the substratum in adult rat cardiomyocytes. J. Cell Biol. 118:14111420 .

Dejana, E., S. Colella, G. Conforti, M. Abbadini, M. Gaboli, and P.C. Marchisio. 1988. Fibronectin and vitronectin regulate the organization of their respective Arg-Gly-Asp adhesion receptors in cultured human endothelial cells. J. Cell Biol. 107:1215-1223.

D’Souza, S.E., M.H. Ginsberg, T.A. Burke, S.C. Lam, and E.F. Plow. 1988. Localization of an Arg-Gly-Asp recognition site within an integrin adhesion receptor. Science. 242:91-93.

Emmert, D.A., J.A. Fee, Z.M. Goeckeler, J.M. Grojean, T. Wakatsuki, E.L. Elson, B.P. Herring, P.J. Gallagher, and R.B. Wyoslmerski. 2004. Rho-kinase-mediated Ca2+-independent contraction in rat embryo fibroblasts. Am. J. Physiol. Cell Physiol. 286:C8-C21.

Eto, M., T. Ohmori, M. Suzuki, K. Furuya, and F. Morita. 1995. A novel protein phosphatase-1 inhibitory protein potentiated by protein kinase $\mathrm{C}$ isolation from porcine aorta media and characterization. J. Biochem. 118:1104-1107.

Ezratty, E.J., M.A. Partridge, and G.G. Gundersen. 2005. Microtubule-induced focal adeshion disassembly is mediated by dynamin and focal adhesion kinase. Nat. Cell Biol. 7:581-590.

Fabry, B., A.H. Klemm, S. Kienle, T.E. Schaffer, and W.H. Goldmann. 2011. Focal adhesion kinase stabilizes the cytoskeleton. Biophys. J. 101:2131-2138.

Fath, K.R., C.J. Edgell, and K. Burridge. 1989. The distribution of distinct integrins in focal contacts is determined by the substratum composition. J. Cell Sci. 92:67-75.

Freedman, A.S., K. Rhynhart, Y. Nojima, J. Svahn, L. Eliseo, C.D. Benjamin, C.D, C. Morimoto, and E. Vivier. 1993. Stimulation of protein tyrosine phosphorylation in human B cells after ligation of the beta 1 integrin VLA-4. J. Immunol. 150:1645-1652.

Frame, M.C., H. Patel, B. Serrels, D. Lietha, and M.J. Eck. 2010. The FERM domain: organizing the structure and function of FAK. Nat. Rev. Mol. Cell Biol. 11:802-814.

Furuta, Y., D. Ilic, S. Kanazawa, N. Takeda, T. Yamamoto, and S. Aizawa. 1995. Mesodermal defect in late phase of gastrulation by a targeted mutation of focal adhesion kinase, FAK.

Oncogene. 11:1989-1995. 
Gabarra-Niecko, V., M.D. Schaller, and J.M. Dunty. 2003. FAK regulates biological processes important for the pathogenesis of cancer. Cancer Metastasis Rev. 22:359-374.

Gallant, N.D., J.R. Capadona, A.B. Frazier, D.M. Collard, and A.J. Garcia. 2002. Micropatterned surfaces for analyzing cell adhesion strengthening. Langmuir. 18:5579-5584.

Geiger, B., D. Salomon, M. Takeichi, and R.O. Hynes. 1992. A chimeric N-cadherin/beta 1integrin receptor which localizes to both cell-cell and cell-matrix adhesions. J. Cell Sci. 103:943-951.

Giannone, G., P. Ronde, M. Gaire, J. Beaudouin, J. Haiech, J. Ellenberg, and K. Takeda. 2004. Calcium rises locally trigger focal adhesion disassembly and enhance residency of focal adhesion kinase at focal adhesions. J. Biol. Chem. 279:28715-28723.

Giuliano, K.A., and D.L. Taylor. 1990. Formation, transport, contraction, and disassembly of stress fibers in fibroblasts. Cell Motil. Cytoskelet. 16:14-21.

Goeckeler, Z.M., and R.B. Wysolmerski. 1995. Myosin light chain kinase-regulated endothelial cell contraction: the relationship between isometric tension, actin polymerization, and myosin phosphorylation. J. Cell Biol. 130:613-627.

Golden, A., J.S. Brugge, and S.J. Shattil. 1990. Role of platelet membrane glycoprotein IIb-IIIa in agonist-induced tyrosine phosphorylation of platelet proteins. J. Cell Biol. 111:3117-3127.

Guan, J-L., J.E. Trevithick, and R.O. Hynes. 1991. Fibronectin/integrin interaction induces tyrosine phosphorylation of a 120-kDa protein. Cell Regul. 2:951-964.

Guo, W.H., M.T. Frey, N.A. Burnham, and Y.L. Wang. 2006. Substrate rigidity regulates the formation and maintenance of tissues. Biophys. J. 90:2213-2220.

Hall, A., 1994. Small GTP-binding proteins and the regulation of the actin cytoskeleton. Annu. Rev. Cell Biol. 10:31-54.

Hanks, S.K., M.B. Calalb, M.C. Harper, and S.K. Patel. 1992. Focal adhesion protein-tyrosine kinase phosphorylated in response to cell attachment to fibronectin. Proc. Natl. Acad. Sci. USA. 89:8487-8491.

Harte, M.T., J.D. Hildebrand, M.R. Burnham, A.H. Bouton, and J.T. Parsons. 1996. p130Cas, a substrate associated with $\mathrm{v}-\mathrm{Src}$ and $\mathrm{v}-\mathrm{Crk}$, localizes to focal adhesions and binds to focal adhesion kinase. J. Biol. Chem. 271:13649-13655.

Hayashi, Y., B. Haimovich, A. Reszka, D. Boettiger, and A. Horwitz. 1990. Expression and function of chicken integrin beta 1 subunit and its cytoplasmic domain mutant in mouse NIH 3T3 cells. J. Cell Biol. 110:175-184. 
Hildebrand, J.D., M.D. Schaller, and J.T. Parsons. 1993. Identification of sequences required for the efficient localization of the focal adhesion kinase, pp125FAK, to cellular adhesions. $J$. Cell Biol. 123:993-1005.

Hildebrand, J.D., M.D. Schaller, and J.T. Parsons. 1995. Paxillin, a tyrosine phosphorylated focal adhesion-associated protein binds to the carboxyl terminal domain of focal adhesion kinase. Mol. Cell Biol. 6:637-647.

Hildebrand, J.D., J.M. Taylor, and J.T. Parsons. 1996. An SH3 domain-containing GTPaseactivating protein for Rho and $\mathrm{Cdc} 42$ associates with focal adhesion kinase. Mol Cell Biol. 16:3169-3178.

Holinstat, M., N. Knezevic, M. Broman, A.M. Samarel, A.B. Malik, and D. Mehta. 2006. Suppression of RhoA activity by focal adhesion kinase-induced activation of p190RhoGAP: role in regulation of endothelial permeability. J. Biol. Chem. 281:2296-2305.

Hynes, R.O. 1992. Integrins: versatility, modulation, and signaling in cell adhesions. Cell. 69:11-25.

Hynes, R.O. 2002. Integrins: bidirectional, allosteric signaling machines. Cell. 110:673-687.

Ichikawa, K., M. Ito, and D.J. Hartshorne. 1996. Phosphorylation of the large subunit of myosin phosphatase and inhibition of phosphatase activity. J. Biol. Chem. 271:4733-4740.

Ilic, D., E.A. Almeida, D.D. Schlaepfer, P. Dazin, S. Aizawa, and C.H. Damsky. 1998. Extracellular matrix survival signals transduced by focal adhesion kinase suppress p53-mediated apoptosis. J. Cell Biol. 143:547-560.

Ilic, D., Y. Furuta, S. Kanazawa, N. Takeda, K. Sobue, N. Nakatsuji, S. Nomura, J. Fujimoto, M. Okada, and T. Yamamoto. 1995. Reduced cell motility and enhanced focal adhesion contact formation in cells from FAK-deficient mice. Nature. 377:539-544.

Ilic, D., B. Kovacic, S. McDonagh, F. Jin, C. Baumbusch, D.G. Gardner, and C.H. Damsky. 2003. Focal adhesion kinase is required for blood vessel morphogenesis. Circ. Res. 92:300307.

Ishida, T., M.L. Ishida, J. Suero, M. Takahashi, and B.C. Berk. 1999. Agonist-stimulated cytoskeletal reorganization and signal transduction at focal adhesions in vascular smooth muscle cells require c-Src. J. Clin. Invest. 103:789-797.

Ito, M., T. Nakano, F. Erdodi, and D.J. Hartshorne. 2004. Myosin phosphatase: structure, regulation and function. Mol. Cell Biochem. 259:197-209.

Izzard, C.S., and L.R. Lochner. 1976. Cell-to-substrate contracts in living fibroblasts: an interference reflexion study with an evaluation of the technique. J. Cell Sci. 21:129-159. 
Jacamo, R.O., and E. Rozengurt. 2005. A truncated FAK lacking the FERM domain displays high catalytic activity but retains responsiveness to adhesion-mediated signals. Biochem.

Biophys. Res. Commun. 334:1299-1304.

Jockusch, B.M., P. Bubeck, K. Giehl, M. Kroemker, J. Moschner, M. Rothkegel, M. Rudiger, K. Schluter, G. Stanke, and J. Winkler. 1995. The molecular architecture of focal adhesions. Annu. Rev. Cell Dev. Biol. 11:379-416.

Kanner, S.B., L.S. Grosmaire, J.A. Ledbetter, and N.K. Damle. 1993. Beta 2-integrin LFA-1 signaling through phospholipase C-gamma 1 activation. Proc. Natl. Acad. Sci. USA. 90:70997103.

Kanner, S.B., A.B. Reynolds, R.R. Vines, and J.T. Parsons. 1990. Monoclonal antibodies to individual tyrosine-phosphorylated protein substrates of oncogene-encoded tyrosine kinases. Proc. Natl. Acad. Sci. USA. 87:3328-3332.

Kano, Y., K. Katoh, M. Masuda, and K. Fujiwara. 1996. Macromolecular composition of stress fiber-plasma attachment sites in endothelial cells in situ. Circ. Res. 79:1000-1006.

Katz, B.Z., S. Miyamoto, H. Teramoto, M. Zohar, D. Krylov, C. Vinson, J.S. Gutkind, and K.M. Yamada. 2002. Direct transmembrane clustering and cytoplasmic dimerization of focal adhesion kinase initiates its tyrosine phosphorylation. Biochim. Biophys. Acta. 1592:141-152.

Kawano, Y., Y. Fukata, N. Oshiro, M. Amaon, T. Nakamura, M. Ito, F. Matsumura, M. Inagaki, and K. Kaibuchi. Phosphorylation of myosin-binding subunit (MBS) of myosin phosphatase by Rho-kinase in vivo. J. Cell Biol. 147:1023-1038.

Kimura, K., M. Ito, M. Amano, K. Chihara, Y. Fukata, M. Nakafuku, B. Yamamori, J. Feng, T. Nakano, K. Okawa, A. Iwamatsu, and K. Kaibuchi. 1996. Regulation of myosin phosphatase by Rho and Rho-associated kinase (Rho-kinase). Science. 273:245-248.

Klemm, A.H., G. Diez, J-L. Alonso, and W.H. Goldmann. 2009. Comparing the mechanical influence of vincluin, focal adhesion kinase and p53 in mouse embryonic fibroblasts. Biochem. Biophys. Res. Commun. 379:799-801.

Kollmannsberger, P., and B. Fabry. High-force magnetic tweezers with force feedback for biological applications. Rev. Sci. Instrum. 78:114301-114306.

Kornberg, L., H.S. Earp, J.T. Parsons, M. Schaller, and R.L. Juliano. 1992. Cell adhesion or integrin clustering increases phosphorylation of a focal adhesion-associated tyrosine kinase. $J$. Biol. Chem. 267:23429-23442.

Kornberg, L.J., H.S. Earp, C.E. Turner, C. Prockop, and R.L. Juliano. 1991. Signal transduction by integrins: increased protein tyrosine phosphorylation caused by clustering of beta 1 integrins. Proc. Natl. Acad. Sci. USA. 88:8392-8396. 
Krieg, M., Y. Arboleda-Estudillo, P.H. Puech, J. Kafer, F. Graner, D.J. Muller, and C.P. Heisenberg. 2008. Tensile forces govern germ-layer organization in zebrafish. Nat. Cell Biol. 10:429-436.

Kureishi, Y., S. Kobayashi, M. Amano, K. Kimuar, H. Kanaide, T. Nakano, K. Kaibuchi, and M. Ito. 1997. Rho-associated kinase directly induces smooth muscle contraction through myosin light chain phosphorylation. J. Biol. Chem. 272:12257-12260.

LaFlamme, S.E., L.A. Thomas, S.S. Yamada, and K.M. Yamada. 1994. Single subunit chimeric integrins as mimics and inhibitors of endogenous integrin functions in receptor localization, cell spreading and migration, and matrix assembly. J. Cell Biol. 126:1287-1298.

Lagares, D., O. Busnadiego, R.A. Garcia-Fernandez, M. Kapoor, S. Liu, D.E. Carter, D. Abraham, X. Shi-Wen, P. Carreira, B.A. Fontaine, B.S. Shea, A.M. Tager, A. Leask, S. Lamas, and F. Rodriguez-Pascual. 2012. Inhibition of focal adhesion kinase prevents experimental lung fibrosis and myofibroblast formation. Arthritis Rheum. 64:1653-1654.

Larsen, M., V.V. Artym, J.A. Green, and K.M. Yamada. 2006. The matrix reorganized: extracellular matrix remodeling and integrin signaling. Curr. Opin. Cell Biol. 18:463-471.

Lee, S., A. Zeiger, J.M. Maloney, M. Kotecki, K.J. Van Vliet, and I.M. Herman. 2010. Pericyte actomyosin-mediated contraction at the cell-material interface can modulate the microvascular niche. J. Phys. Condens. Matter. 22:194115.

Leucht, P., J.B. Kim, J.A. Currey, J. Brunski, and J.A. Helms. 2007. FAK-mediated mechanotransduction in skeletal regeneration. PLoS ONE. 2:e390.

Li, S., M. Kim, Y.L. Hu, S. Jalali, D.D. Schlaepfer, T. Hunter, S. Chien, and J.Y. Shyy. 1997. Fluid shear stress activation of focal adhesion kinase. Linking to mitogen-activated protein kinases. J. Biol. Chem. 272:30455-30462.

Lietha, D., X. Cai, D.F. Ceccarelli, Y. Li, M.D. Schaller, and M.J. Eck. 2007. Structural basis for the autoinhibition of focal adhesion kinase. Cell. 129:1177-1187.

Liu, S., D.A. Calderwood, and M.H. Ginsberg. 2000. Integrin cytoplasmic domain-binding proteins. J. Cell Sci. 113:3563-3571.

Liu, Y., J.C. Loijens, K.H. Martin, A.V. Karginov, and J.T. Parsons. 2002. The association of ASAP1, an ADP ribosylation factor-GTPase activating protein, with focal adhesion kinase contributes to the process of focal adhesion assembly. Mol. Biol. Cell. 13:2147-2156.

Lo, C.M., H.B. Wang, M. Dembo, and Y.L. Wang. 2000. Cell movement is guided by the rigidity of the substrate. Biophys. J. 79:144-152. 
Massia, S.P., and J.A. Hubbell. 1991. An RGD spacing of $440 \mathrm{~nm}$ is sufficient for integrin alpha V beta 3-mediated fibroblast spreading and $140 \mathrm{~nm}$ for focal contact and stress fiber formation. J. Cell Biol. 114:1089-1100.

Marcantonio, E.E., J.L. Guan, J.E. Trevithick, and R.O. Hynes. 1990. Mapping of the functional determinants of the integrin beta 1 cytoplasmic domain by site-directed mutagenesis. Cell Regul. 1:597-604.

Michael, K.E., D.W. Dumbauld, K.L. Burns, S.E. Hanks, and A.J. Garcia. 2009. Focal adhesion kinase modulates cell adhesion strengthening via integrin activation. Mol. Biol. Cell. 20:25082519.

Miyamoto, S., S.K. Akiyama, and K.M. Yamada. 1995a. Synergistic roles for receptor occupancy and aggregation in integrin transmembrane function. Science. 267:883-885.

Miyamoto, S., H. Teramoto, O.A. Coso, J.S. Gutkind, P.D. Burbelo, S.K. Akiyama, and K.M. Yamada. 1995b. Integrin function: molecular hierarchies of cytoskeletal and signaling molecules. J. Cell Biol. 131:791-805.

Nobes, C.D., and A. Hall. 1999. Rho GTPases control polarity, protrusion, and adhesion during cell movement. J. Cell Biol. 144:1235-1244.

Nojima, Y., D.M. Rothstein, K. Sugita, S.F. Schlossman, and C. Morimoto. 1992. Ligation of VLA-4 on T cells stimulates tyrosine phosphorylation of a 105-kD protein. J. Exp. Med. 175:1045-1053.

Owen, J.D., P.J. Ruest, D.W. Fry, and S.K. Hanks. 1999. Induced focal adhesion kinase (FAK) expression in FAK-null cells enhances cell spreading and migration requiring both auto- and activation loop phosphorylation sites and inhibits adhesion-dependent tyrosine phosphorylation of Pyk2. Mol. Cell Biol. 19:4806-4818.

Parsons, J.T. 1993. Focal adhesion kinase: the first ten years. J. Cell Sci. 116:1409-1416.

Pasapera, A.M., I.C. Schneider, E. Rericha, D.D. Schlaepfer, and C.M. Waterman. 2010. Myosin II activity regulates vinculin recruitment to focal adhesions through FAK-mediated paxillin phosphorylation. J. Cell Biol. 188:877-890.

Peng, X., X. Wu, J.E. Druso, H. Wei, A.Y. Park, M.S. Kraus, A. Alcaraz, J. Chen, S. Chien, R.A. Cerione, and J.L. Guan. 2008. Cardiac developmental defects and eccentric right ventricular hypertrophy in cardiomyocyte focal adhesion kinase (FAK) conditional knockout mice. Proc. Natl. Acad. Sci. USA. 105:6638-6643.

Pirone, D.M., W.F. Liu, S.A. Ruiz, L. Gao, S. Raghaven, C.A. Lemmon, L.H. Romer, and C.S. Chen. 2006. An inhibitory role for FAK in regulating proliferation: a link between limited adhesion and RhoA-ROCK signaling. J. Cell Biol. 174:277-288. 
Polte, T.R., and S.K. Hanks. 1995. Interaction between focal adhesion kinase and Crkassociated tyrosine kinase substrate p130Cas. Proc. Natl. Acad. Sci. USA. 92:10678-10682.

Poullet, P., A. Gautreau, G. Kadare, J.A. Girault, D. Louvard, and M. Arpin. 2001. Ezrin interacts with focal adhesion kinase and induces its activation independently of cell-matrix adhesion. J. Biol. Chem. 276:37686-37691.

Puklin-Faucher, E., and M.P. Sheetz. 2009. The mechanical integrin cycle. J. Cell Sci. 122(Pt 2):179-186.

Ren, X.D., W.B. Kiosses, and M.A. Schwartz. 1999. Regulation of the small GTP-binding protein Rho by cell adhesion and the cytoskeleton. EMBO J. 18:578-585.

Ren, X.D., W.B. Kiosses, D.J. Sieg, C.A. Otey, D.D. Schlaepfer, and M.A. Schwartz. 2000. Focal adhesion kinase suppresses Rho activity to promote focal adhesion turnover. J. Cell Sci. 113:3673-3678.

Renshaw, M.W., L.S. Price, and M.A. Schwartz. 1999. Focal adhesion kinase mediates the integrin signaling requirement for growth factor activation of MAP kinase. J. Cell Biol. 147:611-618.

Richardson, A., and J.T. Parsons. 1995. Signal transduction through integrins: a central role for focal adhesion kinase? Bioessays. 17:229-236.

Richardson, A., and J.T. Parsons. 1996. A mechanism for regulation of the adhesion-associated protein tyrosine kinase pp125FAK. Nature. 380:538-540,

Rico, B., H.E. Beggs, D. Schahin-Reed, N. Kimes, A. Schmidt, and L.F. Reichardt. 2004. Control of axonal branching and synapse formation by focal adhesion kinase. Nat. Neurosci. 7:1059-1069.

Ridley A.J., and A. Hall. 1992. The small GTP-binding protein rho regulates the assembly of focal adhesions and actin stress fibers in response to growth factors. Cell. 70:389-399. Ruoslahti, E. 1991. Integrins. J. Clin. Invest. 87:1-5.

Riveline, D., E. Zamir, N.Q. Balaban, U.S. Schwarz, T. Ishizaki, S. Narumiya, Z. Kam, B. Geiger, and A.D. Bershadsky. 2001. Focal contacts as mechanosensors: externally applied local mechanical force induces growth of focal contacts by an mDia1-dependent and ROCKindependent mechanism. J. Cell Biol. 153:1175-1186.

Ruoslahti, E. 1991. Integrins. J. Clin. Invest. 87:1-5.

Saez, A., A. Buguin, P. Silberzan, and B. Ladoux. 2005. Is the mechanical activity of epithelial cells controlled by deformations or forces? Biophys. J. 89:L52-54 
Sastry, S.K., and K. Burridge. 2000. Focal adhesions: a nexus for intracellular signaling and cytoskeletal dynamics. Exp. Cell Res. 261:25-36.

Sawada, Y., and M.P. Sheetz. 2002. Force transduction by Triton cytoskeletons. J. Cell Biol. 156:609-615.

Schaller, M.D., C.A. Borgman, B.S. Cobb, R.R. Vines, A.B. Reynolds, and J.T. Parsons. 1992. pp125FAK, a structurally distinctive protein-tyrosine kinase associated with focal adhesions. Proc. Natl. Acad. Sci. USA. 89:5192-5196.

Schaller, M.D., C.A. Borgman, and J.T. Parsons. 1993. Autonomous expression of a noncatalytic domain of the focal adhesion-associated protein tyrosine kinase pp125FAK. Mol. Cell Biol. 13:785-791.

Schaller, M.D., J.D. Hildebrand, and J.T. Parsons. 1999. Complex formation with focal adhesion kinase: A mechanism to regulate activity and subcellular localization of Src kinases. Mol. Biol. Cell. 10:3489-3505.

Schaller, M.D., J.D. Hildebrand, J.D Shannon, J.W. Fox, R.R. Vines, and J.T. Parsons. 1994. Complex formation with focal adhesion kinase: Autophosphorylation of the focal adhesion kinase pp125FAK directs SH2-dependent binding of pp60src. Mol. Biol. Cell. 14:1680-1688.

Schaller, M.D., C.A. Otey, J.D. Hildebrand, and J.T. Parsons. 1995. Focal adhesion kinase and paxillin bind to peptides mimicking beta integrin cytoplasmic domains. J. Cell Biol. 130:11811187.

Schaller, M.D., and J.T. Parsons. 1994. Focal adhesion kinase and associated proteins. Curr. Opin. Cell Biol. 6:705-710.

Schaller, M.D., and J.T. Parsons. 1995. pp125FAK-dependent tyrosine phosphorylation of paxillin creates a high-affinity binding site for Crk. Mol. Cell Biol. 15:2635-2645.

Schober, M., S. Raghaven, M. Nikolova, L. Polak, H.A. Pasolli, H.E. Beggs, L.F. Reichardt, and E. Fuchs. 2007. Focal adhesion kinase modulates tension signaling to control actin and focal adhesion dynamics. J. Cell Biol. 176:667-680.

Scholey, J.M., K.A. Taylor, and J. Kendrick-Jones. 1980. Regulation of non-muscle myosin assembly by calmodulin-dependent light chain kinase. Nature. 287:233-235.

Schwartz, M.A. 2010. Integrins and extracellular matrix in mechanotransduction. Cold Spring Harb. Perspect. Biol. doi: 10.1101/cshperspect.a005066.

Sellers, J.R. 1991. Regulation of cytoplasmic and smooth muscle myosin. Curr. Opin. Cell Biol. 3:98-104. 
Seufferlein, T., and E. Rozengurt. 1994. Lysophosphatidic acid stimulates tyrosine phosphorylation of focal adhesion kinase, paxillin, and p130. Signaling pathways and cross-talk with platelet-derived growth factor. J. Biol. Chem. 269:9345-9351.

Sharp, W.W., D.G. Simpson, T.K. Borg, A.M. Samarel, and L. Terracio. 1997. Mechanical forces regulate focal adhesion and costamere assembly in cardiac myocytes. Am. J. Physiol. 273:H546-556.

Shen, T.L., A.Y. Park, A. Alcaraz, X. Peng, I. Jang, P. Koni, R.A. Flavell, H. Gu, and J.L. Guan. 2005. Conditional knockout of focal adhesion kinase in endothelial cells reveals its role in angiogenesis and vascular development in late embryogenesis. J. Cell Biol. 169:941-952.

Shen, Y., and M.D. Schaller. 1999. Focal adhesion targeting: the critical determinant of FAK regulation and substrate phosphorylation. Mol. Biol. Cell. 10:2507-2518.

Sheppard, D. 2000. In vivo functions of integrins: lessons from null mutations in mice. Matrix Biol. 19:203-209.

Shimizu, H., M. Ito, M. Miyahara, K. Ichikawa, S. Okubo, T. Konishi, M. Naka, T. Tanaka, K. Hirano, D.J. Hartshorne, and T. Nakano. 1994. Characterization of the myosin-binding subunit of smooth muscle myosin phosphatase. J. Biol. Chem. 269:30407-30411.

Shirazi, A., K. Iizuka, P. Fadden, C. Mosse, A.P. Somlyo, A.V. Somlyo, and T.A. Haystead. 1994. Purification and characterization of the mammalian myosin light chain phosphatase holoenzyme. The differential effects of the holoenzyme and its subunits on smooth muscle. $J$. Biol. Chem. 269:31598-31606.

Sieg, D.J., C.R. Hauck, D. Ilic, C.K. Klingbeil, E. Schaefer, C.H. Damsky, and D.D. Schlaepfer. 2000. FAK integrates growth-factor and integrin signals to promote cell migration. Nat. Cell Biol. 2:249-256.

Sieg, D.J., C.R. Hauck, and D.D. Schlaepfer. 1999. Required role of focal adhesion kinase (FAK) for integrin-stimulated cell migration. J. Cell Sci. 112:2677-2691.

Simpson, D.G., M.L. Decker, W.A. Clark, and R.S. Decker. 1993. Contractile activity and cellcell contact regulate myofibrillar organization in cultured cardiac myocytes. J. Cell Biol. 123:323-336.

Singer, I.I., S. Scot, D.W. Kawka, D.M. Kazazis, J. Gailit, and E. Ruoslahti. 1988. Cell surface distribution of fibronectin and vitronectin receptors depends on substrate composition and extracellular matrix accumulation. J. Cell Biol. 106:2171-2182.

Sinnett-Smith, J., I. Zachary, A.M. Valverde, and E. Rozengurt. 1993. Bombesin stimulation of p125 focal adhesion kinase tyrosine phosphorylation. Role of protein kinase C, Ca2+ mobilization, and the actin cytoskeleton. J. Biol. Chem. 268:14261-14268. 
Solowska, J., J.L. Guan, E.E. Marcantonio, J.E. Trevithick, C.A. Buck, and R.O. Hynes. 1989. Expression of normal and mutant avian integrin subunits in rodent cells. J. Cell Biol. 109:853861.

Sumi, T., K. Matsumoto, Y. Takai, and T. Nakamura. 1999. Cofilin phosphorylation and actin cytoskeletal dynamics regulated by rho- and Cdc42-activated LIM-kinase 2. J. Cell Biol. 147:1519-1532.

Tachibana, K., T. Sato, N. D’Avirro, and C. Morimoto. 1995. Direct association of pp125FAK with paxillin, the focal adhesion-targeting mechanism of pp125FAK. J. Exp. Med. 182:10891099.

Takai, Y., T. Sasaki, K. Tanaka, and H. Nakanishi. 1995. Rho as a regulator of the cytoskeleton. Trends Biochem. Sci. 20:227-231.

Tan, J.L., S. Ravid, and J.A. Spudich. 1992. Control of nonmuscle myosins by phosphorylation. Annu. Rev. Biochem. 61:721-759.

Tan, J.L., J. Tien, D.M. Pirone, D.S. Gray, K. Bhadriraju, and C.S. Chen. 2003. Cells lying on a bed of microneedles: An approach to isolate mechanical force. Proc. Natl. Acad. Sci. USA. 100:1484-1489.

Tang, D.D., and S.J. Gunst. 2001. Depletion of focal adhesion kinase by antisense depresses contractile activation of smooth muscle. Am. J. Physiol. Cell Physiol. 280:C874-C883.

Tang, D., D. Mehta, and S.J. Gunst. 1999. Mechanosensitive tyrosine phosphorylation of paxillin and focal adhesion kinase in tracheal smooth muscle. Am. J. Physiol. Cell Physiol. 276:C250-C258.

Taylor, J.M., J.D. Hildebrand, C.P. Mack, M.E. Cox, and J.T. Parsons. 1998. Characterization of graf, the GTPase-activating protein for rho associated with focal adhesion kinase. Phosphorylation and possible regulation by mitogen-activated protein kinase. J. Biol. Chem. 273:8063-8070.

Taylor, J.M., M.M. Macklem, and J.T. Parsons. 1999. Cytoskeletal changes induced by GRAF, the GTPase regulator associated with focal adhesion kinase, are mediated by Rho. J. Cell Sci. 112:231-242.

Thomas, J.W., M.A. Cooley, J.M. Broome, R. Salgia, J.D. Griffin, C.R. Lombardo, and M.D. Schaller. 1999. The role of focal adhesion kinase binding in the regulation of tyrosine phosphorylation of paxillin. J. Biol. Chem. 274:36684-36692.

Tidball, J.G. 1983. The geometry of actin filament-membrane associations can modify adhesive strength of the myotendinous junction. Cell Motil. 3:439-447. 
Tidball, J.G. 1984. Myotendinous junction: morphological changes and mechanical failure associated with muscle cell atrophy. Exp. Mol. Pathol. 40:1-12.

Tidball, J.G., and T.L. Daniel. 1986. Myotendinous junctions of tonic muscle cells: structure and loading. Cell Tiss. Res. 245:315-322.

Tilghman, R.W., J.K. Slack-Davis, N. Sergina, K.H. Martin, M. Iwanicki, E.D. Hershey, H.E. Beggs, L.F. Reichardt, and J.T. Parsons. 2005. Focal adhesion kinase is required for the spatial organization of the leading edge in migrating cells. J. Cell Sci. 118:2613-2623.

Torsoni, A.S., S.S. Constancio, W. Nadruz, S.K. Hanks, and K.G. Franchini. 2003. Focal adhesion kinase is activated and mediates the early hypertrophic response to stretch in cardiac myocytes. Circ. Res. 93:140-147.

Totsukawa, G., Y. Yamakita, S. Yamashiro, D.J. Hartshorne, Y. Sasaki, and F. Matsumura. 2000. Distinct roles of ROCK (Rho-kinase) and MLCK in spatial regulation of MLC phosphorylation for assembly of stress fibers and focal adhesions in 3 T3 fibroblasts. J. Cell Biol. 150:797-806.

Trinkle-Mulcahy, L., K. Ichikawa, D.J. Hartshorne, M.J. Siegman, and T.M. Butler. 1995. Thiophosphorylation of the $130-\mathrm{kDa}$ subunit is associated with a decreased activity of myosin light chain phosphatase in alpha-toxin-permeabilized smooth muscle. J. Biol. Chem. 270:18191-18194.

Trotter, J.A., K. Corbett, and B. Avner. 1981. Structure and function of the murine muscletendon junction. Anat. Rec. 201:293-302.

Trotter, J.A., S. Eberhard, and A. Samora. 1983. Structural connections of the muscle-tendon junction. Cell Motil. 3:431-438.

Turner, C.E., N. Kramarcy, R. Sealock, and K. Burridge. 1991. Localization of paxillin, a focal adhesion protein, to smooth muscle dense plaques, and the myotendinous and neuromuscular junctions of skeletal muscle. Exp. Cell Res. 192:651-655.

van der Flier, A., and A. Sonnenberg. 2001. Function and interactions of integrins. Cell Tissue Res. 305:285-298.

van Nimwegen, M.J., S. Verkoeijen, L. van Buren, D. Burg, and B. van de Water. 2005. Requirement for focal adhesion kinase in the early phase of mammary adenocarcinoma lung metastasis formation. Cancer Res. 65:4698-4706.

Vouret-Craviari, V., P. Boquet, J. Pouyssegur, and E. Van Obberghen-Schilling. 1998. Regulation of the actin cytoskeleton by thrombin in human endothelial cells: role of Rho proteins in endothelial barrier function. Mol. Biol. Cell. 9:2639-2653. 
Wang, H.B., M. Dembo, S.K. Hanks, and Y.Y. Wang. 2001. Focal adhesion kinase is involved in mechanosensing during fibroblast migration. Proc. Natl. Acad. Sci. USA. 98:11295-11300.

Watanabe, F., T. Miyazaki, T. Takeuchi, M. Fukaya, T. Nomura, S. Noguchi, H. Mori, K. Sakimura, M. Watanabe, and M. Mishina. 2008. Effects of FAK ablation on cerebellar foliation, Bergmann glia positioning and climbing fiber territory on Purkinje cells. Eur. J. Neurosci. 27:836-854.

Webb, D.J., K. Donais, L.A. Whitmore, S.M. Thomas, C.E. Turner, J.T. Parsons, and A.F. Horwitz. 2004. FAK-Src signalling through paxillin, ERK and MLCK regulates adhesion disassembly. Nat. Cell Biol. 6:154-161.

Webb, D.J., J.T. Parsons, and A.F. Horwitz. 2002. Adhesions assembly, disassembly and turnover in migrating cells - over and over and over again. Nat. Cell Biol. 4:E97-E100.

Wilson, D.P., C. Sutherland, M.A. Borman, J.T. Deng, J.A. Macdonald, and M.P. Walsh. 2005. Integrin-linked kinase is responsible for $\mathrm{Ca} 2+-$-independent myosin diphosphorylation and contraction of vascular smooth muscle. Biochem. J. 392:641-648.

Wong, V.W., K.C. Rustad, S. Akaishi, M. Sorkin, J.P. Glotzbach, M. Januszyk, E.R. Nelson, K. Levi, J. Paterno, I.N. Vial, A.A. Kuang, M.T. Longaker, and G.C. Gurtner. 2011. Focal adhesion kinase links mechanical force to skin fibrosis via inflammatory signaling. Nat. Med. 18:148-152.

Wozniak, M., A. Fausto, C.P. Carron, D.M. Meyer, and K.A. Hruska. 2000. Mechanically strained cells of the osteoblast lineage organize their extracellular matrix through unique sites of alphavbeta3-integrin expression. J. Bone Miner. Res. 15:1731-1745.

Wu, C., J. Bauer, R. Juliano, and J. McDonald. 1993. The alpha 5 beta 1 integrin fibronectin receptor, but not the alpha 5 cytoplasmic domain, functions in an early and essential step in fibronectin matrix assembly. J. Biol. Chem. 268:21883-21888.

Wu, C., P. Hughes, M. Ginsberg, and J. McDonald. 1996. Identification of a new biological function for the integrin alpha $\mathrm{V}$ beta 3: initiation of fibronectin matrix assembly. Cell Adhes. Commun. 4:149-158.

Wu, X., K.G. Morgan, C.J. Jones, R.M. Tribe, and M.J. Taggart. 2008. Myometrial mechanoadaptation during pregnancy: implications for smooth muscle plasticity and remodeling. J. Cell Mol. Med. 12:1360-1373.

Yang, J.T., and R.O. Hynes. 1996. Fibronectin receptor functions in embryonic cells deficient in alpha 5 beta 1 integrin can be replaced by alpha V integrins. Mol. Biol. Cell. 7:1737-1748.

Yang, N., O. Higuchi, K. Ohashi, K. Nagat, A. Wada, K. Kangawa, E. Nishida, and K. Mizuno. 1998. Cofilin phosphorylation by LIM-kinase 1 and its role in Rac-mediated actin reorganization. Nature. 393:809-812. 
Yano, H., Y. Mazaki, K. Kurokawa, S.K. Hanks, M. Matsuda, and H. Sabe. 2004. Roles played by a subset of integrin signaling molecules in cadherin-based cell-cell adhesion. J. Cell Biol. 166:283-295.

Zaidel-Bar, R., and B. Geiger. 2010. The switchable integrin adhesome. J. Cell Sci. 123:13851388.

Zamir, E., and B. Geiger. 2001. Molecular complexity and dynamics of cell-matrix adhesions. J. Cell Sci. 114:3583-3590.

Zhai, J., H. Lin, Z. Nie, J. Wu, R. Canete-Soler, W.W. Schlaepfer, and D.D. Schalepfer. 2003. Direct interaction of focal adhesion kinase with p190RhoGEF. J. Biol. Chem. 278:2486524873.

Zhao, J.H., H. Reiske, and J.L. Guan. 1998. Regulation of the cell cycle by focal adhesion kinase. J. Cell Biol. 143:1997-2008. 

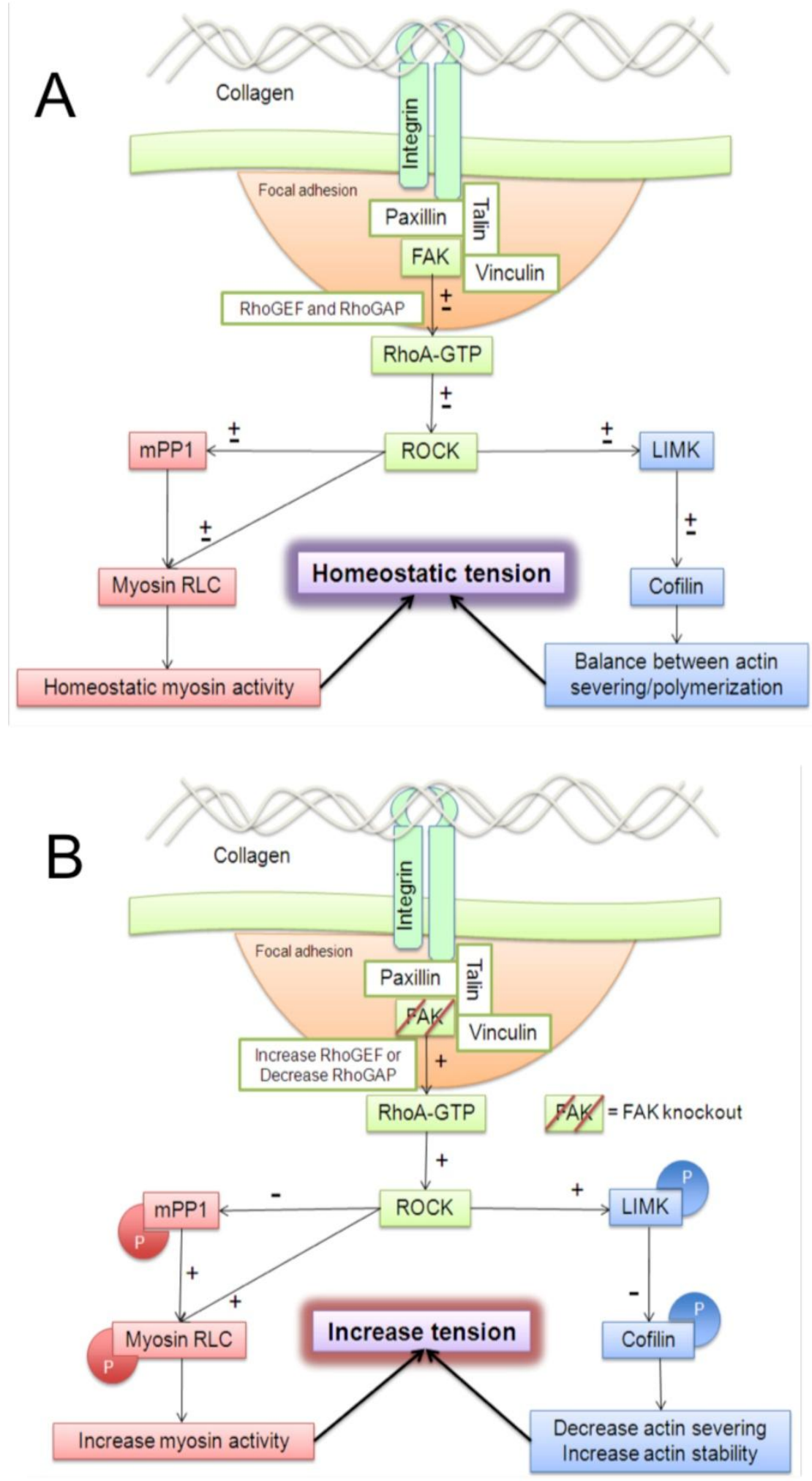

Figure 1 
Figure 1: Model of FAK's involvement in the regulation of cellular tension.

A) Under normal conditions, FAK regulates the activity of RhoA through upstream regulators of GTP binding (i.e. RhoGAPs and RhoGEFs). A balance between active/inactive myosin II and actin severing/polymerization controlled by ROCK leads to the development of basal tension. B) The current accepted model based on data from FAK knockout and knockdown studies indicates that loss of FAK results in changes in the normal balance of RhoA activity, resulting in an increase in active RhoA and leads to activation of ROCK. ROCK can increase myosin II activity and promote actin stabilization resulting in an increase in the generation of cellular tension. 


\title{
Chapter 2
}

\section{Loss of focal adhesion kinase enhances endothelial barrier function and increases focal adhesions}

\author{
Kimberly M. Arnold ${ }^{\mathrm{a}, \mathrm{d}}$, Zoe M. Goeckeler ${ }^{\mathrm{b}, \mathrm{d}}$, and Robert B. Wysolmerski ${ }^{\mathrm{b}, \mathrm{c}, \mathrm{d} \dagger}$ \\ Departments of Physiology and Pharmacology ${ }^{\mathrm{a}}$, Neurobiology and Anatomy ${ }^{\mathrm{b}}$, Mary Babb \\ Randolph Cancer Center ${ }^{\mathrm{c}}$ and Center for Cardiovascular and Respiratory Sciences ${ }^{\mathrm{d}}$, West \\ Virginia University School of Medicine, Morgantown WV 26506
}

Running title: FAK Modulates Endothelial Barrier Function

\section{Grant Numbers and support:}

This research was supported by NIH grants: HL-45788, HL-90937, P20-RR16440 and Cardiovascular and Pulmonary Disease T32 HL-090610.

Corresponding Author ${ }^{\dagger}$ :

Robert Wysolmerski, Ph.D.

Department of Neurobiology and Anatomy

One Medical Center Drive

PO Box 9128

West Virginia University School of Medicine

Morgantown, WV 26506

Tel: $304-293-2213$

FAX: 304-293-5513

Email: rwysolmerski@hsc.wvu.edu

This manuscript was accepted for publication in Microcirculation on April 16, 2013. doi: $10.1111 /$ micc.12063 


\begin{abstract}
Objective: To determine the role of focal adhesion kinase (FAK) in the regulation of endothelial barrier function.
\end{abstract}

Methods: Stable FAK knockdown human pulmonary microvessel endothelial cells were generated by lentiviral infection of FAK shRNA. Measurements of isometric tension and transendothelial electrical resistance were performed.

Results: A FAK knockdown human pulmonary endothelial cell line was generated by lentiviral infection with FAK shRNA and resulted in greater than $90 \%$ reduction in FAK protein with no change in Pyk2 protein. Loss of FAK altered cell morphology and actin distribution in both preand post-confluent endothelial cells. Large, polygonal shaped endothelial cells with randomly organized stress fibers were identified in pre-confluent cultures while in confluent monolayers, endothelial cells were irregularly shaped with actin bundles present at cell margins. An increase in the number and size of vinculin plaques was detected in FAK depleted cells. FAK knockdown monolayers generated a greater transendothelial electrical resistance than controls. Thrombin treatment induced similar changes in transendothelial resistance in both FAK knockdown and control cell lines. FAK depleted endothelial cells developed a higher stable basal isometric tension compared to control monolayers, but the increase in tension stimulated by thrombin does not differ between the cell lines. Basal myosin II regulatory light chain phosphorylation was unaltered in FAK depleted cells. In addition, loss of FAK enhanced VE-cadherin localization to the cell membrane without altering VE-cadherin protein levels.

Conclusion: The loss of FAK in endothelial cells enhanced cell attachment and strengthened cell-cell contacts resulting in greater basal tension leading to formation of a tighter endothelial monolayer.

Key Words: focal adhesions, endothelial cells, isometric tension, permeability 


\section{List of Abbreviations:}

ECIS - electric cell-substrate impedance sensing system

ECM - extracellular matrix

FAK - focal adhesion kinase

FAK-KO - focal adhesion kinase knockout mouse embryonic fibroblasts

FAK-WT - wild-type mouse embryonic wild-type fibroblasts

HLEC - human pulmonary microvessel endothelial cells

FAK-KD - focal adhesion kinase knockdown human pulmonary microvessel endothelial cells

MEFs - mouse embryonic fibroblasts

RLC - myosin II regulatory light chain

TER - transendothelial resistance

WT - wild-type control human pulmonary microvessel endothelial cells 


\section{Introduction}

Endothelial cells lining blood vessels form a continuous layer that confines proteins and blood elements to the vascular lumen. Disruption of the continuous endothelial barrier leads to an increase in permeability and development of edema, a hallmark of acute and chronic inflammation (Lucas et al., 2009; Dushianthan et al., 2011; Mac et al., 2011). Mechanisms that may play a role in altering barrier function leading to edema formation include: 1) loss of endothelial cell cohesion, 2) opening of endothelial cell junctions, 3) activation of intrinsic contractile activity, and 4) disruption of the constitutive balance between homotypic cohesion, junctional integrity, and basal cellular tension.

The interaction of endothelial cells with extracellular matrix (ECM) proteins is equally important for maintenance and stabilization of blood vessels. Endothelial cells attach to the ECM through transmembrane receptors, integrins, which coalesce in formation of focal adhesions (Burridge and Chrzanowska-Wodnicka, 1996). These structures are specialized sites of adhesion that connect the ECM with the cytoskeleton providing the structural link between intra- and extracelluar compartments. This connection allows for efficient transmission of biochemical signals and isometric tension from within the cell to its extracellular microenvironment. Intracellular tension develops when myosin II interacts with actin which is anchored to focal adhesions. The magnitude of tension generated has been shown to correlate with the number and size of focal adhesions (Riveline et al., 2001; Bershadsky et al., 2003; Tan et al., 2003). Thus, maintaining the selective barrier function(s) of the endothelium requires a structural and functional balance between cell-cell and cell matrix interactions.

Several studies have documented the role of endothelial cell contractile activity in edema formation (Shasby et al., 1982; Wysolmerski et al., 1984; Wysolmerski and Lagunoff, 1985); 
however, the role of focal adhesions in endothelial barrier function remains unclear. FAK has emerged as an important regulatory enzyme in many endothelial cell functions ( $\mathrm{Wu}, 2005$; Vadali et al., 2007; Yuan and Rigor, 2010; Yuan et al., 2012). FAK is a cytoplasmic nonreceptor tyrosine kinase (Kornberg et al., 1992; Schaller et al., 1992), which has been shown to be important in the development of the cardiovascular system (Shen et al., 2005; Braren et al., 2006; Zhao et al., 2010). Studies targeting FAK deletion in endothelial cells have detected blood vessel malformations, which contribute to embryonic lethality (Shen et al., 2005), while studies examining FAK's role in vascular permeability have disparate results. Several groups have shown that FAK activation enhances endothelial barrier function (Belvitch and Dudek, 2012; Quadri, 2012), while other reports suggest FAK activation contributes to endothelial barrier dysfunction. Thus, the role of FAK in vascular permeability remains unclear. The purpose of this study was to investigate the role of FAK in vascular permeability using pulmonary microvascular endothelial cells in culture. A stable FAK knockdown human pulmonary microvascular endothelial cell line was established and used to assess the effect of FAK deletion on endothelial cell permeability, basal and agonist induced isometric tension, the morphological distribution of actin and vinculin, and myosin II phosphorylation.

\section{Methods}

\section{Cell Culture}

Mouse embryonic wild-type (FAK-WT) and FAK knockout (FAK-KO) fibroblasts were obtained from ATCC (Manassas, VA) and maintained as outlined previously (Ilic et al., 1995). Immortalized human pulmonary microvessel endothelial cells (HLEC) (Shao and Guo, 2004) were obtained from Dr. Shao (Biomedical Research Institute, University of Massachusetts at 
Amherst) and maintained in EGM2-MV media supplemented with 20\% FCS (Sigma-Aldrich, St. Louis, MO). All cells were grown at $37^{\circ} \mathrm{C}, 5 \% \mathrm{CO}_{2}$.

\section{Generation of FAK Knockdown Endothelial Cells}

Human FAK knockdown (FAK-KD) endothelial cells were generated using a CMV driven GIPZ lentiviral vector which bicistronically encodes GFP and an shRNA to the coding region within the catalytic core of human FAK (Clone \#V2LHS_57330; Open Biosystems, Thermo Scientific, Waltham, MA). Lentivirus was generated by transfecting subconfluent $293 \mathrm{~T}$ cells with the FAK shRNA lentiviral vector and $2^{\text {nd }}$ generation lentiviral packaging vectors, psPAX2 and pCMVVSVG (3:2:1: ratio of DNA concentration, respectively). cDNAs were mixed with TurboFect transfection agent (Thermo Scientific, Waltham, MA) and cells were transfected as outlined previously (Dull et al., 1998; Wang and McManus, 2009). Supernatants containing viral particles were collected every 12 hours for 96 hours, supernatants combined and centrifuged at 2,000 rpm for 10 minutes at $4^{\circ} \mathrm{C}$. Viral supernatants were filtered through a $0.45 \mu \mathrm{m}$ low protein binding syringe filter, frozen, and stored at $-80^{\circ} \mathrm{C}$. To generate FAK-KD HLECs, HLECs were seeded at a density of $4.2 \times 10^{5}$ cells/T- 25 flask 24 hours prior to the addition of FAK-shRNA lentivirus. After 72 hours, shRNA-FAK virus was removed and cells were re-fed in complete growth media. After reaching confluence, cells were split and incubated in the presence of 5 $\mu \mathrm{g} / \mathrm{ml}$ puromycin to select for infected cells. The GIPZ lentiviral vector carries a resistance gene for puromycin allowing only infected cells to survive in the presence of puromycin. Since infected endothelial cells bicistronically express GFP, puromycin-treated cells were also sorted by flow cytometry to isolate a stable FAK-KD cell line. Following cell sorting, stable FAK-KD cells were continuously kept in the presence of $5 \mu \mathrm{g} / \mathrm{ml}$ puromycin for maintaining a stable 
FAK-KD HLEC line. FAK protein levels were measured by Western blot and GFP expression was confirmed using confocal microscopy. To confirm results obtained with the Open Biosystems lenti construct, a limited number of experiments were performed using a stable shRNA FAK knockdown HLEC cell line generated by targeting the 3'-UTR of FAK mRNA (AGCATTGGGTCGGGAACTAA) (Bryant et al., 2012).

\section{Immunofluorescence}

For fluorescent staining, cells were grown in $35 \mathrm{~mm}$ collagen coated dishes. Cells were fixed, permeabilized, and immunostained as outlined by Goeckeler and Wysolmerski (1995). For vinculin staining, cells were incubated with a 1:1,500 dilution of a monoclonal mouse antivinculin antibody (Sigma-Aldrich, St. Louis, MO; Cat.\#-V9131), for VE-cadherin staining 2 $\mu \mathrm{g} / \mathrm{ml}$ of a mouse monoclonal anti-VE cadherin antibody (Santa Cruz Biotechnology Inc., Santa Cruz, CA; Cat.\#-sc9989) and to visualize actin filaments, cultures were incubated with rhodamine phalloidin (Sigma-Aldrich, St. Louis, MO, Cat.\#-P1951). Cells were examined using a Zeiss 510 laser confocal microscope.

\section{Western Blot Analysis}

For western blots, monolayers were lysed in $75 \mu 1$ of SDS sample buffer and protein determinations were performed using a Pierce BCA protein assay after chloroform/methanol extraction as outlined by Wessel and Flugge (1984). Cell lysates $(32 \mu \mathrm{g})$ were electrophoresed on 10\% SDS PAGE gels as described previously (Goeckeler and Wysolmerski, 1995; Goeckeler and Wysolmerski, 2005). PVDF membranes were incubated with the following antibodies: 1:100,000 dilution mouse monoclonal anti-GAPDH (Fitzgerald Industries International, Acton 
MA; Cat.\#-10R-G109A); 1:1,000 dilution of rabbit polyclonal anti-FAK, 1:800 dilution of rabbit anti-Pyk2, 1:500 dilution of mouse monoclonal caspase-3, 1:1,000 dilution of mouse monoclonal N-cadherin (13A9) (Santa Cruz Biotechnology Inc., Dallas, TX; Cat.\#sc-558, Cat.\#sc-9019, Cat.\#sc-7272, Cat.\#sc-59987, respectively), 1:1,000 dilution of rabbit polyclonal anti-cleaved caspase-3 (Asp175) (Cell Signaling Technology, Danvers, MA; Cat.\#9661), 1:1,000 dilution of monoclonal mouse anti-vinculin antibody (Sigma-Aldrich, St. Louis, MO; Cat.\#V9131), and 1:15,000 dilution of mouse monoclonal VE-cadherin Hec1 Cad5 overnight at $4^{\circ} \mathrm{C}$. For visualization of proteins, membranes were incubated in a 1:8,000 dilution of HRP-conjugated goat anti-rabbit or goat anti-mouse (Bio-Rad Laboratories, Hercules, CA) antibodies for 1 hour at RT, washed with TBS/0.1\% Tween-20, and incubated in ECL reagents (GE Healthcare, Piscataway, NJ). Blots were exposed on X-Ray films to detect signal emitted from protein bands. Protein levels were quantitated by laser densitometry (Goeckeler and Wysolmerski, 1995; Chew et al., 1998). GAPDH was used as loading control in all Western blots. Protein levels were measured by Western blot for three independent experiments.

\section{Measurement of Transendothelial Resistance (TER)}

The effect of FAK knockdown on HLEC permeability was assessed by measurement of TER using Applied BioPhysics Model 1600R Electric Cell-Substrate Impedance Sensing System (ECIS) (Applied BioPhysics, Troy, NY). ECIS electrode arrays (8W10E+) were obtained from Applied BioPhysics, cleaned with $10 \mathrm{mM}$ cysteine, and coated with $5 \mu \mathrm{g} / \mathrm{ml}$ rat tail type I collagen in $0.15 \mathrm{M} \mathrm{NaCl}$ for $12 \mathrm{hrs}$ at RT. Electrodes were washed with $300 \mu \mathrm{l}$ complete media, electro-pulsed stabilized, and equilibrated at $37^{\circ} \mathrm{C}, 5 \% \mathrm{CO}_{2}$ for 2 hours. Arrays were inoculated with $1 \times 10^{5}$ cells/well in a total volume of $400 \mu 1$ and immediately connected to the electrode 
clamps. Electrical resistance was continuously measured until a stable TER was detected (approximately 60 hours). To assess changes in agonist stimulated monolayer permeability, 10 $\mathrm{nM}$ thrombin was added to each well and the change in electrical resistance monitored for the duration of the experiment. A drop in electrical resistance correlates with a breakdown in cellcell/cell-matrix interactions (i.e. leaky monolayer) while an increase in resistance indicates development of a more cohesive monolayer (i.e. tighter monolayer, less leak). Data were collected at 1 minute intervals, and resistance values (ohms) were plotted as a function of time (hours). WT and FAK-KD tracings are an average of data from 6 individual wells \pm s.e.m. Experiments are representative of similar experiments conducted at least nine additional times.

\section{Myosin Phosphorylation and Isometric Tension Measurements}

To determine basal levels of myosin II regulatory light chain phosphorylation (RLC), cells were seeded onto collagen coated 35-mm dishes and the extent of myosin RLC phosphorylation analyzed as outlined previously (Goeckeler et al., 2000; Brown et al., 2009). Briefly, phosphorylated myosin II RLC was detected by the ECL method (Amersham Corp., Arlington Heights, IL) using an affinity-purified rabbit antibody generated to phospho Ser-19 RLC. X-ray films were developed at various time intervals to obtain an exposure within the linear range of the film. Films were scanned in a personal densitometer (Molecular Dynamics, Sunnyvale, CA) in two dimensions; the densitometry units (DU) for each sample were standardized based on total myosin II RLC content (see below) and expressed as a ratio of densitometric units to total RLC content. Results were then expressed as relative phospho-RLC content (P-RLC); i.e., PhosphoRLC to Total-RLC (P-RLC = Phospho-RLC/ Total-RLC). 
Total RLC content was determined by probing Western blots with affinity-purified RLC antibodies raised against recombinant nonmuscle myosin II RLC as outlined previously (Chew et al., 1998). Total myosin II RLC were detected by ECL methodology and analyzed as outlined above. Phosphorylation experiments were repeated three times.

Isometric tension measurements were performed as described in detail by Goeckeler et al. (Goeckeler and Wysolmerski, 1995; Goeckeler and Wysolmerski, 2005; Goeckeler et al., 2008).

\section{Results}

A stable FAK knockdown (FAK-KD) endothelial cell line was generated by infecting pulmonary microvessel endothelial cells with a lentivirus expressing an shRNA targeted to human FAK. FAK protein levels in stable FAK-KD endothelial cells were reduced by $85-90 \%$ of those in control (WT) endothelial cell as shown in Figure 1. In FAK knockout fibroblasts (Sieg et al., 1998; Lim et al., 2008) and endothelial cells (Weis et al., 2008), a closely related protein, Pyk2, has been shown to be up-regulated compensating for the loss of FAK. Pky2 levels were unchanged in FAK-KD endothelial cells, indicating Pyk2 does not compensate for loss of FAK (Figure 1). FAK protein levels were routinely monitored by western blots to ensure FAK knockdown was consistent between experiments. Studies overexpressing a naturally occurring

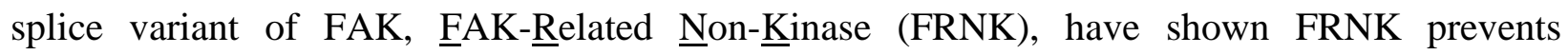
phosphorylation of endogenous FAK inhibiting cell proliferation and survival. Furthermore, studies on endothelial cells from knockout mice have reported conflicting results on FAK's role in proliferation, migration and survival (Braren et al., 2006; Zhao et al., 2010; Chen et al., 2012). To ensure our stable FAK-KD cell line did not inhibit cell proliferation or alter cell survival, we performed cell growth studies, assessed cell death by trypan blue exclusion, and determined if 
loss of FAK induced apoptotic cell death. We found depletion of FAK had minimal effect on cell proliferation (Figure 2A) and cell death (data not shown) in agreement with the results published by Bryant et al. (2012). In addition, FAK-KD endothelial cells showed no activation of caspase-3 (Figure 2B) or significant annexin $\mathrm{V}$ staining (data not shown) indicating loss of FAK did not induce apoptotic cell death.

Electric cell-substrate impedance sensing (ECIS) was used to measure transendothelial resistance (TER) of WT and FAK-KD endothelial cell monolayers as a measure of endothelial cell adherence, cell spreading, and monolayer permeability. As shown in Figure 3A, FAK-KD endothelial cells developed a higher TER when compared to control monolayers. For the first 15 minutes post-seeding, WT and FAK-depleted endothelial cells generated a similar electrical resistance profile, which suggests that loss of FAK does not affect cell attachment (Figure 3B). TER in FAK depleted cells continued to rapidly increase to a 1.3-fold level above controls by 90 minutes (Figure 3B). Resistance steadily increased over the duration of the experiment (approximately 60 hours) in FAK-KD endothelial cells reaching a stable TER 1.4-fold higher than WT controls (Figure 3A). Resistance in WT endothelial cell monolayers developed slower and never attained the same resistance levels (cohesiveness) as FAK-KD endothelial cells (Figure 3A). To determine if the difference in electrical resistance was due to variation in WT vs FAK-KD cell number, the DNA content (Emmert et al., 2004; Goeckeler et al., 2008) in each ECIS well was determined at the end of an experiment. WT and FAK-KD endothelial cell DNA content from ECIS wells were comparable $(870 \pm 0.391$ vs $897 \pm 0.307$ ngDNA, respectively) indicating there was no significant difference in WT and FAK-KD cell growth within ECIS wells. These results were consistent with our cell proliferation data (Figure 2) and indicate the higher electrical resistance generated by FAK-KD cells was not due to more cells per well. 
Following establishment of stable TER, ECIS arrays were removed from clamps, cells refed with complete media, and arrays reattached to the ECIS apparatus. As shown in Figure 3C, FAK-KD endothelial cells generated and maintained a higher stable TER than WT control (76 hours post-seeding) and TER was unaltered by addition of fresh media.

Exposure to $10 \mathrm{nM}$ thrombin induced a rapid drop in electrical resistance in both WT and FAK-KD endothelial cell monolayers (Figure 3D). Recovery of TER was similar in both cell lines; however, control monolayers developed a higher TER post-recovery than do FAK-KD endothelial cell monolayers (Figure 3D). Taken together, these data suggest the initial differences in resistance results from differential cell size and/or spreading while the development of a higher basal TER indicates FAK depletion induces formation of tighter more cohesive endothelial cell monolayers (Figure 3A).

We next sought to determine if the enhancement of the barrier function was accompanied by changes in F-actin and/or focal adhesion distribution. WT endothelial cells seeded at preconfluent densities exhibited an elongate morphology with actin stress fibers, which run parallel to one another and traverse the length of the cell (Figure 4A). In contrast, FAK-KD endothelial cells were twice the size and have a polygonal shape (WT endothelial cells, $6,794 \pm 161 \mu \mathrm{m}^{2}$ vs FAK-KD endothelial cells, $13,284 \pm 286 \mu \mathrm{m}^{2}$; Table1) with actin stress fibers arranged parallel to one another at the cell margins. In the cell center, actin bundles were shorter and ran in several directions. No peripheral rim of actin stress fibers was detected. To visualize focal adhesions, indirect immunofluorescence labeling of vinculin, a major focal adhesion protein was undertaken. Small vinculin plaques were located throughout WT endothelial cell cytoplasm, occupying approximately $0.16 \%$ of the total cell area (Table 1 ). In FAK-KD endothelial cells, an 
increase in size and number of vinculin plaques was detected resulting in a 1.5-fold increase in total vinculin plaque area (Table 1, Figure 4A).

Major contributions to the knowledge of FAK's role in actin dynamics and focal adhesion localization come from experiments done with FAK-KO mouse embryo fibroblasts (MEFs) (Ilic et al., 1995). To determine if our results were comparable to the established FAKKO MEF model, a limited number of localization studies were performed on FAK-KO and FAK-WT MEFs. FAK-KO MEFs seeded at pre-confluent densities were small, round cells compared to FAK-WT MEFs (Figure 4B). Rhodamine phalloidin staining revealed most FAKKO MEFs exhibited a prominent peripheral band of actin filaments with short thick randomly dispersed central stress fibers (Figure 4B). FAK-WT MEFs exhibit long parallel stress fibers running the length of the cell (Figure 4B). Focal adhesions in FAK-WT MEFs were small, round plaques localized at the cell periphery while in FAK-KO MEFs there was an increase in size and number of large elongate plaques compared to FAK-WT MEFs (Figure 4B) as previously reported (Ilic et al., 1995; Ren et al., 2000). Similar changes in vinculin distribution were found in FAK-depleted endothelial cells and FAK-KO fibroblasts, however, FAK's effects on cell shape and size was unique to each cell type.

We next sought to determine if the F-actin distribution and focal adhesion content changed in confluent monolayers similar to that seen in pre-confluent endothelial cells. Figure 4C shows a representative confocal micrograph of WT and FAK-KD endothelial cell monolayers. WT monolayers consist of a cohesive sheet of endothelial cells with actin filaments traversing the long axis of the cell. In contrast, cells in FAK knockdown monolayers were irregular in shape with short actin filaments that run perpendicular to one another. The majority of vinculin plaques in control monolayers were localized at the cell margins as small discrete 
plaques. Approximately $1 \%$ of the cell area was occupied by vinculin plaques in WT endothelial cell monolayers (Table 1). In FAK knockdown monolayers, a 52\% increase (Table 1) in vinculin plaque area was detected. The number and size of plaques increased throughout the cytoplasm and at cell margins (Figure 4C). No difference in the cellular vinculin protein content was detected between WT and FAK-KD cell lines (Figure 5) suggesting loss of FAK results in either a reduction in focal adhesion turnover (Quadri et al., 2003) and/or reorganization and recruitment of cytoplasmic vinculin to new and existing adhesion plaques. Taken together, we speculate loss of FAK induced an increase in number and size of focal adhesion plaques, strengthening cell-cell contacts, and attachment to the extracellular matrix, leading to formation of tighter monolayers.

Focal adhesions are the specialized structures that connect the ECM with the cytoskeleton providing the conduit for generation of isometric tension. Previously, we had shown that endothelial cell monolayers generated a stable basal isometric tone associated with a low level of myosin II RLC phosphorylation (Goeckeler et al., 2008). Since a greater number of vinculin plaques are present in FAK-KD endothelial cells, we speculated cells producing more focal adhesions would generate a greater basal endothelial cell tone (Riveline et al., 2001; Bershadsky et al., 2003; Tan et al., 2003). To explore this possibility, the effect of FAK-KD on basal and agonist-induced isometric tension was determined on HLEC monolayers as described previously (Goeckeler and Wysolmerski, 1995; Emmert et al., 2004). Figure 6A is a representative isometric tension tracing of thrombin-stimulated WT and FAK-KD endothelial cell monolayers. FAK-KD monolayers consistently developed a higher basal tension (20-25 dynes) compared to control monolayers. However, upon $10 \mathrm{nM}$ thrombin stimulation, loss of FAK appeared to have little effect on agonist induced tension generation. Both knockdown and control monolayers 
responded to thrombin with a rapid rise in tension development achieving approximately the same peak tension. After achieving peak tension, the decline in force was similar in both cell lines. Addition of $2 \mu \mathrm{M}$ cytochalasin D rapidly abolished isometric tension produced by the endothelial cell monolayers.

Reports have shown FAK-KO MEFs exhibit a higher basal myosin II RLC phosphorylation than FAK-WT MEFs when seeded onto fibronectin coated dishes (Chen et al., 2002; Pirone et al., 2006). To determine if the loss of FAK effects the extent of endothelial cell myosin II RLC phosphorylation, FAK-KD endothelial cells and WT endothelial cells were seeded onto collagen-coated dishes and the extent of myosin II RLC phosphorylation assessed as described previously (Brown et al., 2009; Goeckeler et al., 2008). In both FAK-depleted and WT endothelial cell monolayers, $20 \%$ of the myosin II RLC was mono-phosphorylated with no significant amount of di-phosphorylated RLC detected in either cell line (Figure 6B). In addition, no significant difference in myosin II RLC phosphorylation could be detected in thrombin-treated monolayers (data not shown). Thus, the extent of endothelial cell basal myosin II RLC phosphorylation was not affected by the loss of FAK as observed in FAK-KO MEFs (Chen et al., 2002; Pirone et al., 2006).

Cadherins are essential homotypic adhesion molecules that specifically localize to adherens junctions and control endothelial cell integrity (Dejana et al., 2009; Harris and Nelson, 2010). Since loss of FAK has been associated with changes in cadherin distribution (Zhao et al., 2010), we sought to determine if FAK depletion altered the content and localization of endothelial cell cadherins. Western blot analysis showed both VE- and N-cadherin protein levels did not change in FAK-KD endothelial cells compared to WT endothelial cells (Figure 7). In both WT and FAK depleted monolayers, VE-cadherin immuno-localized to intercellular 
junctions outlining the borders between adjacent cells. VE-cadherin exhibited an irregular or jagged pattern at many inter-endothelial cell junctions due to co-localization with stress fibers terminating at sites of cell-cell contact (Figure 8A). No increase in cytoplasmic VE-cadherin staining was detected in FAK depleted monolayers (Figure 8G). Although, VE-cadherin protein levels were comparable in both cell lines (Figure 7) the intensity of VE-cadherin staining was greater at cell margins in FAK depleted monolayers compared to WT monolayers (Figure 8A and G). Together, these data suggest loss of FAK increases the recruitment of VE-cadherin from cytoplasmic pools to sites of cell-cell contact.

Consistent with previously published studies, thrombin treatment induced endothelial cell retraction/contraction (Goeckeler and Wysolmerski, 1995; Rabiet et al., 1996; Hordijk et al., 1999; Emmert et al., 2004) with formation of gaps between adjacent endothelial cells in both WT and FAK-KD endothelial cell monolayers (Figure 8D and J). The extent of gap formation was greater and more widespread in control monolayers than in FAK depleted cultures. No VEcadherin staining was detected at cell margins that outlined the intercellular gaps in both cell lines (Figure $8 \mathrm{C}$ and $\mathrm{I}$ ). In regions of the monolayers where cells did not retract from one another, VE-cadherin remained localized at cell margins. Restoration of monolayer integrity following thrombin treatment occurred over a two hour period (Figure 8C-F and I-L) with closure of inter-endothelial cell gaps and re-establishment of VE-cadherin staining at resealed intercellular junctions (Figures $8 \mathrm{E}$ and $\mathrm{F}$ and $\mathrm{K}$ and $\mathrm{L}$ ). Interestingly, thrombin treatment induced formation of thicker stress fibers in WT endothelial cells compared to FAK-KD endothelial cells (Figure $8 \mathrm{D}$ and $\mathrm{J}$ ) while, at two hours post treatment, actin stress fibers appeared more abundant in FAK-depleted monolayers than controls (Figure 8F and L). 


\section{Discussion}

Endothelial cells line the wall of all blood vessels and perform a unique role in regulating a variety of physiological processes such as vascular tone, hemostasis, angiogenesis, and leukocyte transmigration. Vascular endothelial cells establish a selective barrier between the vascular and interstitial space and tightly regulate the bidirectional exchange of water and solutes between these compartments. There is a large body of data that suggests the semi-permeable barrier formed by the microvascular endothelium is maintained through a delicate balance between cell-cell interaction, endothelial cell-matrix adhesion, and the generation of endothelial cell basal tension. These interactions tether endothelial cell to endothelial cell as well as endothelial cell to their ECM establishing a conduit for transmission of cellular forces and biochemical signals between cells and their matrix. Inter-endothelial cell tight junctions and adherens junctions have been considered the key structural components responsible for regulating endothelial cell permeability. However, recent studies indicate transmembrane protein complexes known as the focal adhesions, function in endothelial cell barrier regulation by modulating the attachment of the endothelium to its underlying basement membrane. The focal adhesion proteins, FAK and vinculin, are key members of this macromolecular adhesion complex downstream of integrins that regulate the organization of the actin cytoskeleton for transduction of cellular forces from inside to outside of the cell. FAK's involvement in cell signaling cascades is well recognized, however, its role in regulating endothelial cell barrier function and basal tone remains unclear. In the present study, a stable shRNA FAK knockdown pulmonary microvessel endothelial cell line was established to assess the effects of FAK deletion on endothelial cell focal adhesion distribution, paracellular permeability, maintenance of basal tension and agonist induced permeability, and force development. We report that depletion of 
FAK in endothelial monolayers results in i) increase in the number and size of focal adhesions, ii) formation of a tighter more cohesive monolayer, iii) development of a higher basal endothelial cell tension, iv) no alteration in agonist induced permeability or isometric tension development, and v) no delay in reestablishing TER following agonist stimulation. Our findings directly implicate FAK as an important protein in endothelial cells responsible for regulating endothelial permeability and basal isometric tension.

Vascular permeability is regulated by strengthening or weakening of intercellular junctions and several studies have shown an important role for FAK in modulating endothelial cell permeability (Hordijk et al., 1999; Quadri and Bhattacharya, 2007; Belvitch and Dudek, 2012; Quadri, 2012). Cardiac or pulmonary microvessel endothelial cells isolated from FAK knockout mice exhibit increased monolayer permeability compared to control monolayers. These studies confirmed data showing inhibition or depletion of FAK altered in vitro monolayer permeability of endothelial cells isolated from several vascular beds (Mehta et al., 2002; Quadri et al., 2003; Wu et al., 2003; Chen et al., 2012). Recent studies have shown endothelial cells isolated from FAK kinase dead knockin mice (Chen et al., 2012) grow to confluence and form a non-permeable barrier to high molecular weight dextrans, suggesting that FAK kinase activity may not be involved in the development and maintenance of basal endothelial cell barrier functions. These studies also documented normal vessel development and density in mice with no hemorrhage/edema formation as occurs in the FAK-KO mouse models (Braren et al., 2006; Corsi et al., 2009; Zhao et al., 2010). However, in tissue culture studies, FAK's involvement in altering endothelial cell permeability differs according to the type of stimulus as well as the origin of endothelial cells used for experiments. FAK kinase activity is required to enhance barrier function upon treatment with KCL (hyper-osmotic conditions; (Quadri et al., 2003)), 
HGF (Birukova et al., 2009b), and OxPAPC (Birukova et al., 2007; Birukova et al., 2009a) while the inflammatory agents TGF-beta (Wu et al., 2003; Lee et al., 2007; Chen et al., 2012), thrombin (Rabiet et al., 1996; Shikata et al., 2003; van Nieuw Amerongen et al., 2004), and VEGF (Wu et al., 2003; Chen et al., 2012) activate FAK kinase activity leading to increases in monolayer permeability. In agreement with our studies, Chen et al. (2012) recently reported inhibition of FAK kinase activity with inhibitor PF-262,271 increased basal TER in human pulmonary endothelial cells. Furthermore, studies from intact microvessels have shown increased FAK kinase activity correlates with increases in permeability in response to histamine and VEGF (Wu et al., 2003; Guo et al., 2005; Chen et al., 2012). Thus, FAK has the ability to modulate endothelial cell barrier function both positively and negatively depending on the stimulus involved. Our studies revealed FAK-KD endothelial cells have a higher basal TER compared to control monolayers. We believe the higher basal TER in FAK-KD endothelial cells develops due to the formation a greater number of larger focal adhesion plaques which strengthen endothelial cell-ECM interactions. Previous reports have shown reduction in FAK content or expression of kinase deficient FAK mutants resulted in a prolonged barrier disruption and inhibition of junctional resealing upon agonist stimulation (Rabiet et al., 1996; Hordijk et al., 1999; Garcia et al., 2000; Mehta et al., 2002; Quadri and Bhattacharya, 2007). However, we observed a rapid decrease in TER following thrombin stimulation in both control and FAK-KD endothelial monolayers which recovers to basal levels within two hours. We attribute these differences to the use of different endothelial cell lines in prior experiments. The effects of FAK inhibition on barrier function may differ depending on the vascular bed and endothelial cell species used (Schnitzer et al., 1994; Gebb and Stevens, 2004). Since we were unable to 
completely knockdown FAK (10\% of control levels), it is conceivable that FAK protein levels were sufficient to maintain its physiological role in regulating vascular homeostasis.

It is generally accepted FAK modulates vascular permeability through one of the following mechanisms: i) FAK activation/inhibition regulates myosin II RLC phosphorylation inducing cell contraction leading to intercellular gap formation, ii) inflammatory mediators activate FAK resulting in disassembly and redistribution of focal adhesions, leading to the formation of gaps and increased permeability, iii) FAK activation induces assembly of junctional complexes resulting in a tighter more cohesive monolayer, and iv) activation of FAK effects downstream signaling cascades that modify adhesion complex proteins resulting in altered barrier function (Wu, 2005; Vadali et al., 2007). The general consensus from studies using FAK-KO MEFs and endothelial cells derived from FAK KO mice suggests loss of FAK causes an increase in myosin II RLC phosphorylation, myosin II activation, and cell contraction. The increase in cell contraction is believed to be responsible for the rounded morphology displayed by FAK-KO MEFs and endothelial cells (Ilic et al., 1995; Chen et al., 2002; Pirone et al., 2006). The contraction model has also been proposed to explain endothelial cell junctional disruption and edema formation associated with FAK activation/inactivation. In this study, we found FAK depletion caused an increase in basal isometric tension without an increase in RLC phosphorylation/myosin activation. However, initial studies undertaken in FAK-KO MEFs (Chen et al., 2002; Pirone et al., 2006; Lim et al., 2008) or KD-EC (Pirone et al., 2006) reported an increase in RLC phosphorylation. It is difficult to compare their studies with ours since these studies analyzed myosin II RLC phosphorylation pattern on pre-confluent cells, while our studies were done with post-confluent endothelial cell monolayers. 
Previous studies from our laboratory have shown that basal tension is associated with low levels of RLC phosphorylation (Goeckeler and Wysolmerski, 1995; Goeckeler et al., 2008) and myosin II functions as a major cross-linking protein in confluent endothelial cell monolayers with the potential for generating tension or pre-stress within the cytoskeleton. We propose that the increase in monolayer tension and enhanced barrier function occurs due to the increase in abundance of focal adhesion plaques creating a stronger structural link between endothelial cells and the ECM. In addition, vinculin recruitment to adhesion plaques stabilizes focal adhesions and the associated actin filaments allowing for more efficient transmission of intracellular tension to the matrix. Several lines of evidence lend credence to this idea. Loss of FAK: i) is associated with large stable focal adhesions (Ilic et al., 1995; Ren et al., 2000) which function as attachment platforms for actin filaments increasing the efficiency for force transduction to the ECM. Several studies have shown that the amount of tension generated correlates with the number and size of focal adhesions (Balaban et al., 2001; Galbraith et al., 2002; Bershadsky et al., 2003; Tan et al., 2003; Rape et al., 2011); ii) alters the structural integrity of matrix adhesion causing a conformational change that results in more efficient force transduction to the ECM; iii) results in recruitment of vinculin to the focal adhesion increasing the strength of adhesion plaques (Galbraith et al., 2002; Mierke et al., 2008; Dumbauld et al., 2010a) as well as crosslinking and stabilizing actin filaments associated with focal adhesions. A strengthened adhesion (Matthews et al., 2004; Michael et al., 2009; Dumbauld et al., 2010a) allows for development of greater cellular pre-stress which is transmitted to the ECM and detected as either an increase in the traction force (Balaban et al., 2001; Rape et al., 2011) or basal isometric tension (Goeckeler and Wysolmerski, 1995; Goeckeler et al., 2008). Dumbauld et al. (2010a, b) has shown a reduction in adhesion strength and contractility occurs upon vinculin depletion or re-expression 
of FAK in MEFs isolated from FAK-KO mice lending support to our hypothesis; and iv) induces activation of downstream signaling cascades altering focal adhesion strength or actin dynamics modulating basal tension and monolayer permeability. Taken together our results suggest that FAK depletion modulates endothelial cell-ECM interactions generating a stronger structural link between endothelial cell-endothelial cell/endothelial cell-ECM altering endothelial cell barrier strength and basal tone.

Vascular integrity and endothelial barrier regulation depends critically on the dynamics of the endothelial cell actin cytoskeleton (Garcia et al., 1995; Dudek and Garcia, 2001). Previous studies in bovine and human pulmonary artery endothelial cells have shown treatment with hepatocyte growth factor and sphingosine 1-phosphate enhances barrier integrity (i.e. observed increase in TER as measured by ECIS). These agents induce formation of a dense band of F-actin at the cell margins and stabilize adherens junctions (Garcia et al., 2001; Liu et al., 2002). We observed a similar enrichment of cortical F-actin in unstimulated FAK-KD monolayers (Figure 8H) compared to WT monolayers (Figure 8B). The increase and stabilization of F-actin at the cell periphery most likely occurs in response to vinculin recruitment to adhesion plaques. In this study and other reports (Garcia et al., 1995; Dudek and Garcia, 2001; Garcia et al., 2001; Liu et al., 2002; Belvitch and Dudek, 2012), the formation of a dense peripheral F-actin network appears to correlate with enhancement of endothelial cell barrier function. Therefore, FAK may function as a regulator of endothelial cell barrier integrity through changes in actin dynamics and cell-cell contacts.

Cell-cell contacts maintain the integrity of the endothelial barrier and alterations in junctional proteins can lead to changes in monolayer permeability. FAK can localize to cell-cell contacts where it interacts with adherens junction proteins to facilitate recovery following 
agonist-induced barrier disruption (Quadri and Bhattacharya, 2007; Knezevic et al., 2009). However, following VEGF stimulation, active FAK can mediate phosphorylation of the adherens junction proteins, leading to the junctional disassembly and subsequent increases in permeability (Chen et al., 2012). In our studies, FAK depletion had no effect on VE- or N-cadherin protein levels and only minimal changes in VE-cadherin distribution detected in control or thrombintreated cultures. Our results do not rule out the possibility that recruitment of these proteins to the cell-cell contact has not been altered or may contribute to changes in barrier functions following the loss of FAK.

Numerous studies have demonstrated an important role for FAK in modulating vascular integrity. In this study, we have shown FAK-KD increases endothelial cell basal tone and enhances endothelial cell barrier function without altering VE-cadherin content/distribution or activitating myosin II dependent contractile pathways. The current work suggests that loss of FAK enhances vascular integrity by promoting formation of vinculin adhesion plaques which stabilize actin creating a tighter, more cohesive monolayer. FAK's physiological role in regulating vascular permeability is still unresolved; however, our studies have documented a role for FAK in regulating basal isometric tension and endothelial permeability. A more in depth understanding of FAK's role in regulating endothelial cell permeability will provide new insight for development of therapeutic agents to modulate the vascular leakage associated with many clinical syndromes.

\section{Acknowledgements}

Imaging experiments and image analysis were performed in the West Virginia University Microscope Imaging Facility, which has been supported by the Mary Babb Randolph Cancer 
Center and NIH grants P20 RR016440, P30 RR032138/GM103488 and P20 RR016477. This research was supported by NIH grants: HL-45788, HL-90937, P20-RR16440 and Cardiovascular and Pulmonary Disease T32 HL-090610. 


\section{References:}

Balaban, N.Q., U.S. Schwarz, D. Riveline, P. Goichberg, G. Tzur, I. Sabanay, D. Mahalu, S. Safran, A. Bershadsky, L. Addadi, and B. Geiger. 2001. Force and focal adhesion assembly: a close relationship studied using elastic micropatterned substrates. Nat. Cell Biol. 3:466-472.

Belvitch, P., and S.M. Dudek. 2012. Role of FAK in S1P-regulated endothelial permeability. Microvasc. Res. 83:22-30.

Bershadsky, A.D., N.Q. Balaban, and B. Geiger. 2003. Adhesion-dependent cell mechanosensitivity. Annu. Rev. Cell Dev. Biol. 19:677-695.

Birukova, A.A., F.T. Arce, N. Moldobaeva, S.M. Dudek, J.G. Garcia, R. Lal, and K.G. Birukov. 2009a. Endothelial permeability is controlled by spatially defined cytoskeletal mechanics: atomic force microscopy force mapping of pulmonary endothelial monolayer. Nanomedicine. $5: 30-41$.

Birukova, A.A., S. Chatchavalvanich, O. Oskolkova, V.N. Bochkov, and K.G. Birukov. 2007. Signaling pathways involved in OxPAPC-induced pulmonary endothelial barrier protection. Microvasc. Res. 73:173-181.

Birukova, A.A., I. Cokic, N. Moldobaeva, and K.G. Birukov. 2009b. Paxillin is involved in the differential regulation of endothelial barrier by HGF and VEGF. Am. J. Respir. Cell Mol. Biol. 40:99-107.

Braren, R., H. Hu, Y.H. Kim, H.E. Beggs, L.F. Reichardt, and R. Wang. 2006. Endothelial FAK is essential for vascular network stability, cell survival, and lamellipodial formation. $J$. Cell Biol. 172:151-162.

Brown, J.A., R.B. Wysolmerski, and P.C. Bridgman. 2009. Dorsal root ganglion neurons react to semaphorin $3 \mathrm{~A}$ application through a biphasic response that requires multiple myosin II isoforms. Mol. Biol. Cell. 20:1167-1179.

Bryant, P.W., Q. Zheng, and K.M. Pumiglia. 2012. Focal adhesion kinase is a phosphoregulated repressor of Rac and proliferation in human endothelial cells. Biol. Open. 1:723-730.

Burridge, K., and M. Chrzanowska-Wodnicka. 1996. Focal adhesions, contractility, and signaling. Annu. Rev. Cell Dev. Biol. 12:463-518.

Chen, B.H., J.T. Tzen, A.R. Bresnick, and H.C. Chen. 2002. Roles of Rho-associated kinase and myosin light chain kinase in morphological and migratory defects of focal adhesion kinasenull cells. J. Biol. Chem. 277:33857-33863.

Chen, X.L., J.O. Nam, C. Jean, C. Lawson, C.T. Walsh, E. Goka, S.T. Lim, A. Tomar, I. Tancioni, S. Uryu, J.L. Guan, L.M. Acevedo, S.M. Weis, D.A. Cheresh, and D.D. Schlaepfer. 2012. VEGF-induced vascular permeability is mediated by FAK. Dev. Cell. 22:146-157. 
Chew, T.L., R.A. Masaracchia, Z.M. Goeckeler, and R.B. Wysolmerski RB. 1998. Phosphorylation of non-muscle myosin II regulatory light chain by p21-activated kinase (gamma-PAK). J. Muscle Res. Cell Motil. 19:839-854.

Corsi, J.M., C. Houbron, P. Billuart, I. Brunet, K. Bouvree, A. Eichmann, J.A. Girault, and H. Enslen. 2009. Autophosphorylation-independent and -dependent functions of focal adhesion kinase during development. J. Biol. Chem. 284:34769-34776.

Dejana, E., E. Tournier-Lasserve, and B.M. Weinstein. 2009. The control of vascular integrity by endothelial cell junctions: molecular basis and pathological implications. Dev. Cell. 16:209221.

Dudek, S.M., and J.G. Garcia. 2001. Cytoskeletal regulation of pulmonary vascular permeability. J. Appl. Physiol. 91:1487-1500.

Dull, T., R. Zufferey, M. Kelly, R.J. Mandel, M. Nguyen, D. Trono, and L. Naldini. 1998. A third-generation lentivirus vector with a conditional packaging system. J. Virol. 72:8463-8471.

Dumbauld, D.W., K.E. Michael, S.K. Hanks, and A.J. Garcia. 2010a. Focal adhesion kinasedependent regulation of adhesive forces involves vinculin recruitment to focal adhesions. Biol. Cell. 102:203-213.

Dumbauld, D.W., H. Shin, N.D. Gallant, K.E. Michael, H. Radhakrishna, and A.J. Garcia. 2010b. Contractility modulates cell adhesion strengthening through focal adhesion kinase and assembly of vinculin-containing focal adhesions. J. Cell Physiol. 223:746-756.

Dushianthan, A., M.P. Grocott, A.D. Postle, and R. Cusack. 2011. Acute respiratory distress syndrome and acute lung injury. Postgrad. Med. J. 87:612-622.

Emmert, D.A., J.A. Fee, Z.M. Goeckeler, J.M. Grojean, T. Wakatsuki, E.L. Elson, B.P. Herring, P.J. Gallagher, and R.B. Wysolmerski. 2004. Rho-kinase-mediated Ca2+-independent contraction in rat embryo fibroblasts. Am. J. Physiol. Cell Physiol. 286:C8-21.

Galbraith, C.G., K.M. Yamada, and M.P. Sheetz. 2002. The relationship between force and focal complex development. J. Cell Biol. 159:695-705.

Garcia, J.G., H.W. Davis, and C.E. Patterson. 1995. Regulation of endothelial cell gap formation and barrier dysfunction: role of myosin light chain phosphorylation. J. Cell Physiol. 163:510-522.

Garcia, J.G., F. Liu, A.D. Verin, A. Birukova, M.A. Dechert, W.T. Gerthoffer, J.R. Bamberg, and D. English. 2001. Sphingosine 1-phosphate promotes endothelial cell barrier integrity by Edg-dependent cytoskeletal rearrangement. J. Clin. Invest. 108:689-701. 
Garcia, J.G., K.L. Schaphorst, A.D. Verin, S. Vepa, C.E. Patterson, and V. Natarajan. 2000. Diperoxovanadate alters endothelial cell focal contacts and barrier function: role of tyrosine phosphorylation. J. Appl. Physiol. 89:2333-2343.

Gebb, S., and T. Stevens. 2004. On lung endothelial cell heterogeneity. Microvasc. Res. 68:112.

Goeckeler, Z.M., P.C. Bridgman, and R.B. Wysolmerski. 2008. Nonmuscle myosin II is responsible for maintaining endothelial cell basal tone and stress fiber integrity. Am. J. Physiol. Cell Physiol. 295:C994-1006.

Goeckeler, Z.M., R.A. Masaracchia, Q. Zeng, T.L. Chew, P. Gallagher, and R.B. Wysolmerski. 2000. Phosphorylation of myosin light chain kinase by p21-activated kinase PAK2. J. Biol. Chem. 275:18366-18374.

Goeckeler, Z.M., and R.B. Wysolmerski. 1995. Myosin light chain kinase-regulated endothelial cell contraction: the relationship between isometric tension, actin polymerization, and myosin phosphorylation. J. Cell Biol. 130:613-627.

Goeckeler, Z.M., and R.B. Wysolmerski. 2005. Myosin phosphatase and cofilin mediate cAMP/cAMP-dependent protein kinase-induced decline in endothelial cell isometric tension and myosin II regulatory light chain phosphorylation. J. Biol. Chem. 280:33083-33095.

Guo, M., M.H. Wu, H.J. Granger, and S.Y. Yuan. 2005. Focal adhesion kinase in neutrophilinduced microvascular hyperpermeability. Microcirculation. 12:223-232.

Harris, E.S., and W.J. Nelson. 2010. VE-cadherin: at the front, center, and sides of endothelial cell organization and function. Curr. Opin. Cell Biol. 22:651-658.

Hordijk, P.L., E. Anthony, F.P. Mul, R. Rientsma, L.C. Oomen, and D. Roos. 1999. Vascularendothelial-cadherin modulates endothelial monolayer permeability. J. Cell Sci. 112(Pt 12):1915-1923.

Ilic, D., Y. Furuta, S. Kanazawa, N. Takeda, K. Sobue, N. Nakatsuji, S. Nomura, J. Fujimoto, M. Okada, and T. Yamamoto. 1995. Reduced cell motility and enhanced focal adhesion contact formation in cells from FAK-deficient mice. Nature. 377:539-544.

Knezevic, N., M. Tauseef, T. Thennes, and D. Mehta. 2009. The G protein betagamma subunit mediates reannealing of adherens junctions to reverse endothelial permeability increase by thrombin. J. Exp. Med. 206:2761-2777.

Kornberg, L., H.S. Earp, J.T. Parsons, M. Schaller, and R.L. Juliano. 1992. Cell adhesion or integrin clustering increases phosphorylation of a focal adhesion-associated tyrosine kinase. $J$. Biol. Chem. 267:23439-23442. 
Lee, Y.H., U.S. Kayyali, A.M. Sousa, T. Rajan, R.J. Lechleider, and R.M. Day. 2007. Transforming growth factor-beta1 effects on endothelial monolayer permeability involve focal adhesion kinase/Src. Am. J. Respir. Cell Mol. Biol. 37:485-493.

Lim, Y., S.T. Lim, A. Tomar, M. Gardel, J.A. Bernard-Trifilo, X.L. Chen, S.A. Uryu, R. CaneteSoler, J. Zhai, H. Lin, W.W. Schlaepfer, P. Nalbant, G. Bokoch, D. Ilic, C. Waterman-Storer, and D.D. Schlaepfer. 2008. PyK2 and FAK connections to p190Rho guanine nucleotide exchange factor regulate RhoA activity, focal adhesion formation, and cell motility. J. Cell Biol. 180:187-203.

Liu, F., K.L. Schaphorst, A.D. Verin, K. Jacobs, A. Birukova, R.M. Day, N. Bogatcheva, D.P. Bottaro, and J.G. Garcia. 2002. Hepatocyte growth factor enhances endothelial cell barrier function and cortical cytoskeletal rearrangement: potential role of glycogen synthase kinase3beta. FASEB J. 16:950-962.

Lucas, R., A.D. Verin, S.M. Black, and J.D. Catravas. 2009. Regulators of endothelial and epithelial barrier integrity and function in acute lung injury. Biochem. Pharmacol. 77:17631772.

Mac, S.R., D.F. McAuley, and M.A. Matthay. 2011. Acute lung failure. Semin. Respir. Crit. Care Med. 32:607-625.

Matthews, B.D., D.R. Overby, F.J. Alenghat, J. Karavitis, Y. Numaguchi, P.G. Allen, and D.E. Ingber. 2004. Mechanical properties of individual focal adhesions probed with a magnetic microneedle. Biochem. Biophys. Res. Commun. 313:758-764.

Mehta, D., C. Tiruppathi, R. Sandoval, R.D. Minshall, M. Holinstat, and A.B. Malik. 2002. Modulatory role of focal adhesion kinase in regulating human pulmonary arterial endothelial barrier function. J. Physiol. 539:779-789.

Michael, K.E., D.W. Dumbauld, K.L. Burns, S.K. Hanks, and A.J. Garcia. 2009. Focal adhesion kinase modulates cell adhesion strengthening via integrin activation. Mol. Biol. Cell. 20:2508-2519.

Mierke, C.T., P. Kollmannsberger, D.P. Zitterbart, J. Smith, B. Fabry, and W.H. Goldmann. 2008. Mechano-coupling and regulation of contractility by the vinculin tail domain. Biophys. J. 94:661-670.

Pirone, D.M., W.F. Liu, S.A. Ruiz, L. Gao, S. Raghavan, C.A. Lemmon, L.H. Romer, and C.S. Chen. 2006. An inhibitory role for FAK in regulating proliferation: a link between limited adhesion and RhoA-ROCK signaling. J. Cell Biol. 174:277-288.

Quadri, S.K. 2012. Cross talk between focal adhesion kinase and cadherins: role in regulating endothelial barrier function. Microvasc. Res. 83:3-11. 
Quadri, S.K., M. Bhattacharjee, K. Parthasarathi, T. Tanita, and J. Bhattacharya. 2003. Endothelial barrier strengthening by activation of focal adhesion kinase. J. Biol. Chem. 278:13342-13349.

Quadri, S.K., and J. Bhattacharya. 2007. Resealing of endothelial junctions by focal adhesion kinase. Am. J. Physiol. Lung Cell Mol. Physiol. 292:L334-L342.

Rabiet, M.J., J.L. Plantier, Y. Rival, Y. Genoux, M.G. Lampugnani, and E. Dejana. 1996. Thrombin-induced increase in endothelial permeability is associated with changes in cell-to-cell junction organization. Arterioscler. Thromb. Vasc. Biol. 16:488-496.

Rape, A.D., W.H. Guo, and Y.L. Wang. 2011. The regulation of traction force in relation to cell shape and focal adhesions. Biomaterials. 32:2043-2051.

Ren, X.D., W.B. Kiosses, D.J. Sieg, C.A. Otey, D.D. Schlaepfer, and M.A. Schwartz. 2000. Focal adhesion kinase suppresses Rho activity to promote focal adhesion turnover. J. Cell Sci. 113(Pt 20):3673-3678.

Riveline, D., E. Zamir, N.Q. Balaban, U.S. Schwarz, T. Ishizaki, S. Narumiya, Z. Kam, B. Geiger, and A.D. Bershadsky. 2001. Focal contacts as mechanosensors: externally applied local mechanical force induces growth of focal contacts by an mDia1-dependent and ROCKindependent mechanism. J. Cell Biol. 153:1175-1186.

Schaller, M.D., C.A. Borgman, B.S. Cobb, R.R. Vines, A.B. Reynolds, and J.T. Parsons. 1992. pp125FAK a structurally distinctive protein-tyrosine kinase associated with focal adhesions. Proc. Natl. Acad. Sci. U.S.A. 89:5192-5196.

Schnitzer, J.E., A. Siflinger-Birnboim, P.J. Del Vecchio, and A.B. Malik. 1994. Segmental differentiation of permeability, protein glycosylation, and morphology of cultured bovine lung vascular endothelium. Biochem. Biophys Res.. Commun. 199:11-19.

Shao, R., and X. Guo. 2004. Human microvascular endothelial cells immortalized with human telomerase catalytic protein: a model for the study of in vitro angiogenesis. Biochem. Biophys. Res. Commun. 321:788-794.

Shasby, D.M., S.S. Shasby, J.M. Sullivan, and M.J. Peach. 1982. Role of endothelial cell cytoskeleton in control of endothelial permeability. Circ. Res. 51:657-661.

Shen, T.L., A.Y. Park, A. Alcaraz, X. Peng, I. Jang, P. Koni, R.A. Flavell, H. Gu, and J.L. Guan JL. 2005. Conditional knockout of focal adhesion kinase in endothelial cells reveals its role in angiogenesis and vascular development in late embryogenesis. J. Cell Biol. 169:941-952.

Shikata, Y., K.G. Birukov, A.A. Birukova, A. Verin, and J.G. Garcia. 2003. Involvement of site-specific FAK phosphorylation in sphingosine-1 phosphate- and thrombin-induced focal adhesion remodeling: role of Src and GIT. FASEB J. 17:2240-2249. 
Sieg, D.J., D. Ilic, K.C. Jones, C.H. Damsky, T. Hunter, and D.D. Schlaepfer. 1998. Pyk2 and Src-family protein-tyrosine kinases compensate for the loss of FAK in fibronectin-stimulated signaling events but Pyk2 does not fully function to enhance FAK- cell migration. EMBO J. 17:5933-5947.

Tan, J.L., J. Tien, D.M. Pirone, D.S. Gray, K. Bhadriraju, and C.S. Chen. 2003. Cells lying on a bed of microneedles: an approach to isolate mechanical force. Proc. Natl. Acad. Sci. U.S.A. 100:1484-1489.

Vadali, K., X. Cai, and M.D. Schaller. 2007. Focal adhesion kinase: an essential kinase in the regulation of cardiovascular functions. IUBMB Life. 59:709-716.

van Nieuw Amerongen, G.P., K. Natarajan, G. Yin, R.J. Hoefen, M. Osawa, J. Haendeler, A.J. Ridley, K. Fujiwara, V.W. van Hinsbergh, and B.C. Berk. 2004. GIT1 mediates thrombin signaling in endothelial cells: role in turnover of RhoA-type focal adhesions. Circ. Res. 94:1041-1049.

Wang, X., and M. McManus. 2009. Lentivirus production. J. Vis. Exp. 32:e1499.

Weis, S.M., S.T. Lim, K.M. Lutu-Fuga, L.A. Barnes, X.L. Chen, J.R. Gothert, T.L. Shen, J.L. Guan, D.D. Schlaepfer, and D.A. Cheresh. 2008. Compensatory role for Pyk2 during angiogenesis in adult mice lacking endothelial cell FAK. J. Cell Biol. 181:43-50.

Wessel, D., and U.I. Flugge. 1984. A method for the quantitative recovery of protein in dilute solution in the presence of detergents and lipids. Anal. Biochem. 138:141-143.

Wu, M.H. 2005. Endothelial focal adhesions and barrier function. J. Physiol. 569:359-366.

Wu, M.H., M. Guo, S.Y. Yuan, and H.J. Granger. 2003. Focal adhesion kinase mediates porcine venular hyperpermeability elicited by vascular endothelial growth factor. J. Physiol. 552:691-699.

Wysolmerski, R., and D. Lagunoff. 1985. Pulmonary edema in adult respiratory distress syndrome. Surv. Synth. Pathol. Res. 4:257-268.

Wysolmerski, R., D. Lagunoff, and T. Dahms. 1984. Ethchlorvynol-induced pulmonary edema in rats. An ultrastructural study. Am. J. Pathol. 115:447-457.

Yuan, S.Y., and R.R. Rigor. 2010. Regulation of Endothelial Barrier Function, Colloquium Series on Integrated Systems Physiology: From Molecule to Function to Disease. D.N. Granger and J.P. Granger, editors. Morgan \& Claypool Life Sciences/San Rafael, CA. Chapter 4.

Yuan, S.Y., Q. Shen, R.R. Rigor, and M.H. Wu. 2012. Neutrophil transmigration, focal adhesion kinase and endothelial barrier function. Microvasc. Res. 83:82-88. 
Zhao, X., X. Peng, S. Sun, A.Y. Park, and J.L. Guan. 2010. Role of kinase-independent and dependent functions of FAK in endothelial cell survival and barrier function during embryonic development. J. Cell Biol. 189:955-965. 
Table 1. Effect of FAK knockdown on vinculin plaques

\begin{tabular}{|l|l|l|l|l|}
\hline & $\begin{array}{l}\text { WT } \\
\text { Pre-confluent } \\
\text { endothelial cells }\end{array}$ & $\begin{array}{l}\text { FAK-KD } \\
\text { Pre-confluent } \\
\text { endothelial cells }\end{array}$ & $\begin{array}{l}\text { WT } \\
\text { Confluent } \\
\text { endothelial cells }\end{array}$ & $\begin{array}{l}\text { FAK-KD } \\
\text { Confluent } \\
\text { endothelial cells }\end{array}$ \\
\hline $\begin{array}{l}\text { Vinculin } \\
\text { staining, \% of } \\
\text { cell area }\end{array}$ & $0.16 \pm 0.05$ & $0.25 \pm 0.07$ & $0.87 \pm 0.04$ & $1.7 \pm 0.09$ \\
\hline $\begin{array}{l}\text { Number of } \\
\text { Vinculin plaques } \\
\text { per cell }\end{array}$ & $193 \pm 57$ & $348 \pm 89$ & ND & ND \\
\hline
\end{tabular}

Results are expressed as the percentage (mean \pm SE) of total cell area positive for vinculin staining. Endothelial cell monolayers were fixed, permeabilized and immunostained for vinculin. Photomicrographs were taken with a Zeiss 510 confocal microscope and were analyzed with Imaris Software as outlined previously (27). 


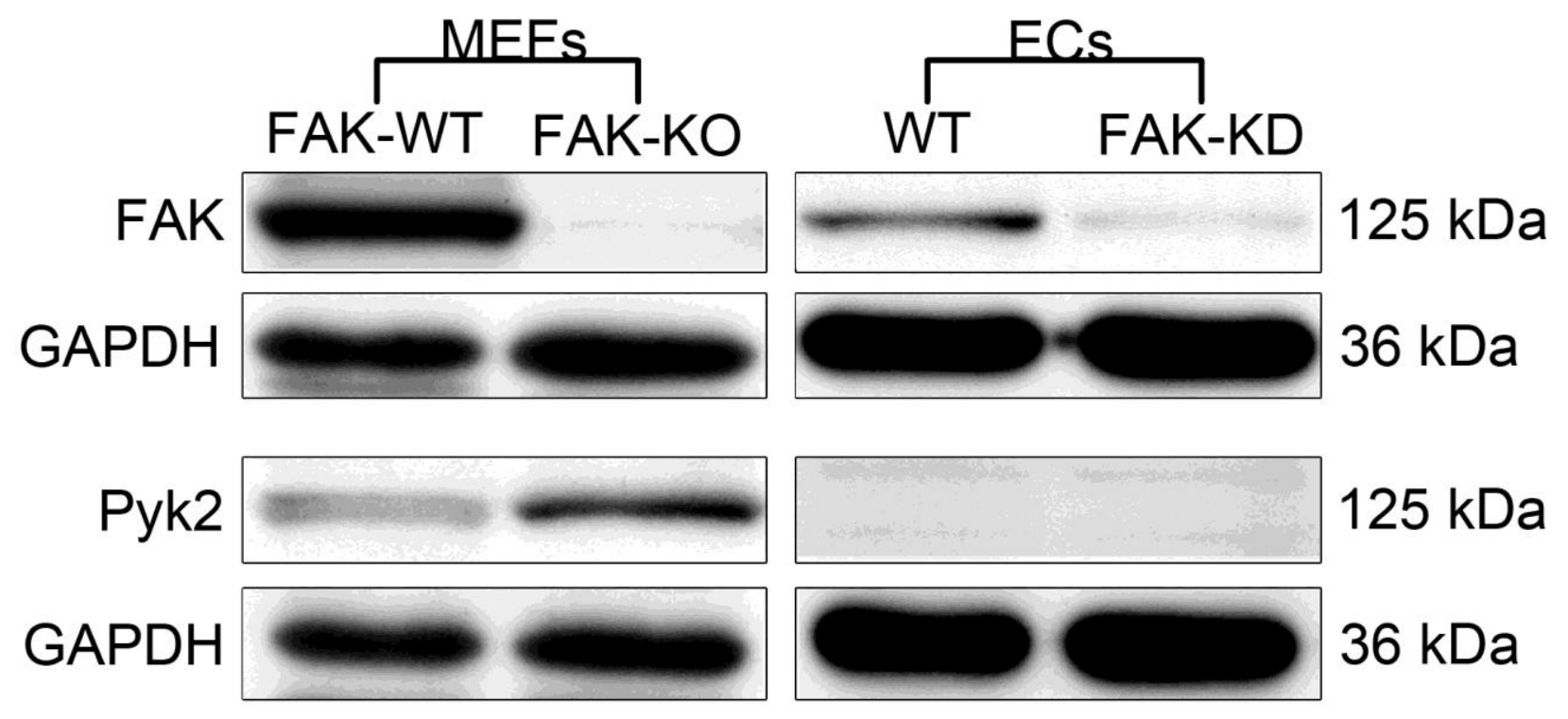

Figure 1 
Figure 1: FAK and Pyk2 protein expression in endothelial cells and MEFs.

Endothelial and MEF cell extracts were analyzed for FAK and Pyk2 protein levels. FAK protein content was knocked down by approximately $80-90 \%$ in stable endothelial cell lines expressing a shRNA targeting FAK. FAK knockdown did not cause up-regulation of endothelial cell Pyk2. GAPDH was used as a loading control. 

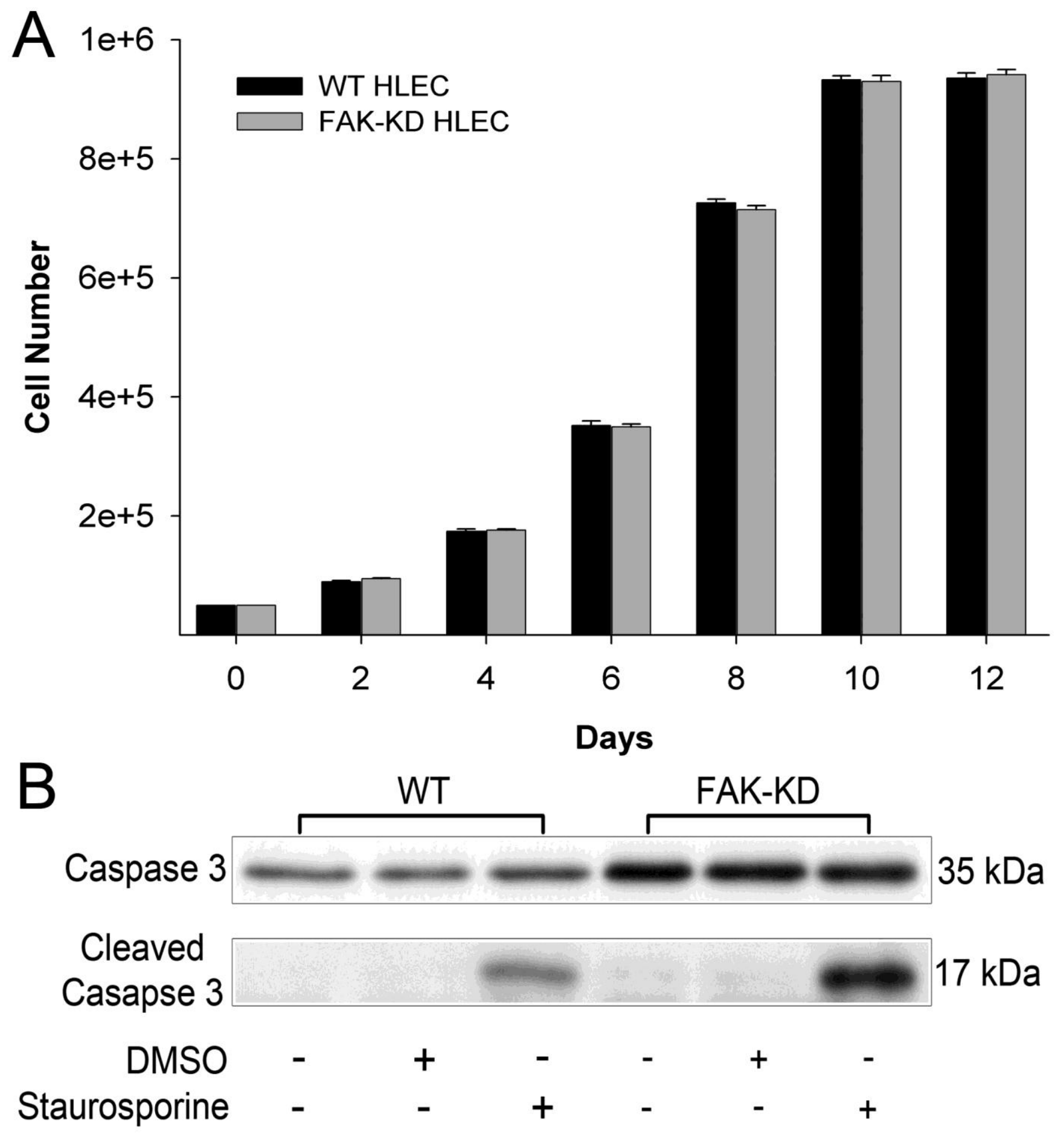

Figure 2 


\section{Figure 2: Effect of FAK knockdown on cell proliferation and death.}

A) Endothelial cells were seeded at subconfluent densities in 6-well plates and cell counts performed every two days for 12 days to compare WT and FAK-KD growth rates. Data plotted are an average of triplicate replicates \pm s.e.m. B) Confluent WT and FAK-KD cells were treated with either $0.1 \mu \mathrm{M}$ DMSO (negative control) or $5 \mu \mathrm{M}$ staurosporine for six hours to activate caspase-3. Western blot analysis of WT and FAK-KD cell extracts reveals treatment of HLEC with staurosporine resulted in activation of caspase 3 in both WT and FAK-KD cells. However, FAK-KD alone does not activate caspase 3. 

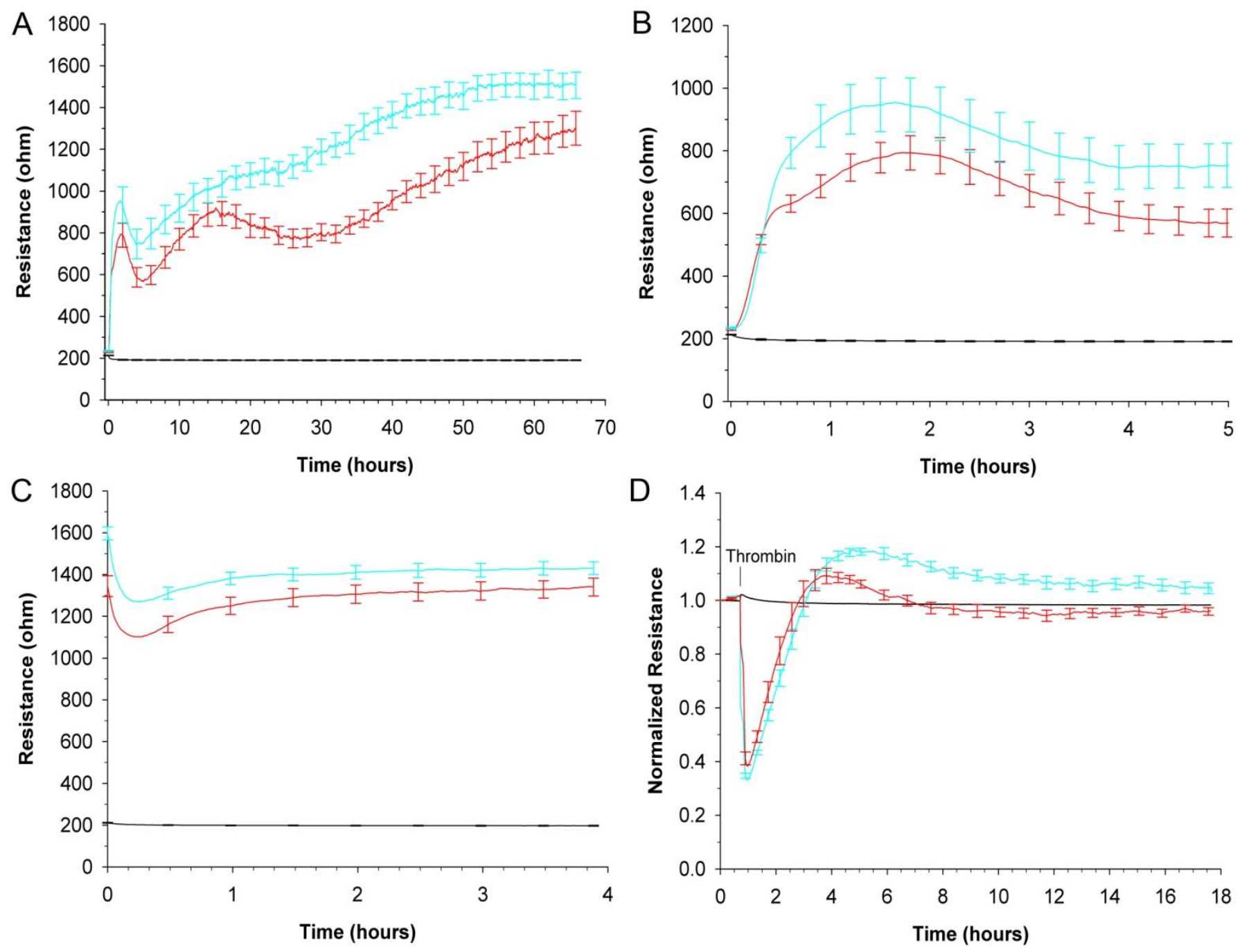

Figure 3 
Figure 3: Effect of FAK knockdown on transendothelial electrical resistance. Representative transendothelial electrical resistance tracing generated by WT and FAK-KD endothelial cells. A) Control (red) and FAK-KD endothelial cells (blue) were plated on gold electrodes and the increase in resistance was followed until endothelial cell monolayers exhibited a stable TER. FAK-KD endothelial cells developed a 1.3-fold higher TER than WT endothelial cell monolayers. B) A typical TER tracing showing FAK-KD endothelial cells generated a greater resistance than WT endothelial cells 15 minutes after seeding. C) Following establishment of stable TER, arrays were removed, cells were re-fed with complete media, and arrays were placed back into the system. TER was recorded for several hours to determine the difference between WT and FAK-KD monolayer basal TER. D) Response of WT and FAK-KD endothelial monolayers to $10 \mathrm{nM}$ thrombin stimulation. WT and FAK-KD tracings are an average of data from 6 individual wells \pm s.e.m. Experiments are representative of similar experiments conducted at least nine additional times. 

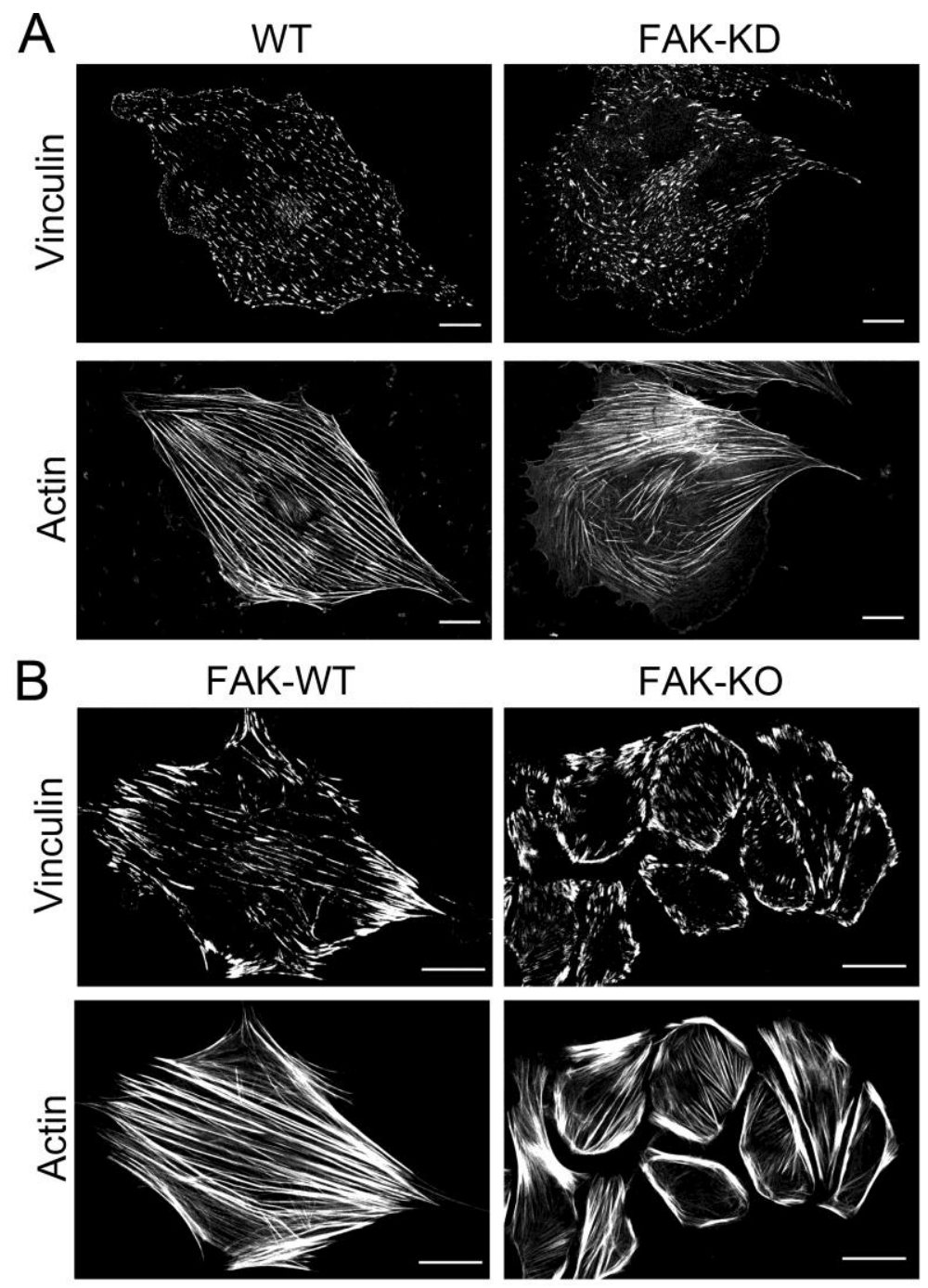

C

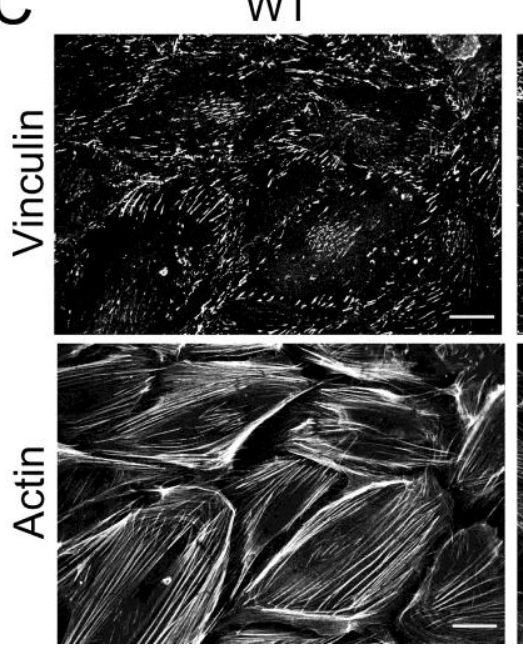

FAK-KD

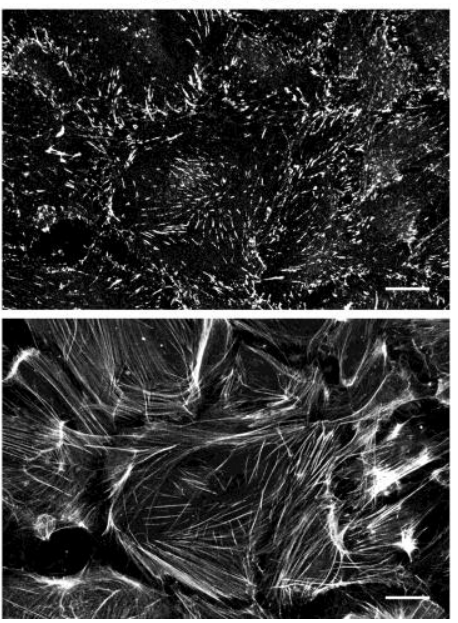

Figure 4 


\section{Figure 4: Localization of actin and vinculin in endothelial cells and MEFs.}

Preconfluent endothelial cells and MEFs were fixed 24 hours after seeding onto collagen-coated dishes, permeabilized, and stained with rhodamine phalloidin to visualize actin filaments and vinculin for localization of focal adhesion plaques. A) Preconfluent endothelial cells: In WT endothelial cells, vinculin was localized in small plaques randomly distributed throughout the cell. Actin was bundled into prominent filaments running the length of the cell. FAK depletion induced larger prominent vinculin plaques while actin localization was disorganized. Bar $20 \mu \mathrm{m}$. B) Preconfluent MEFs: FAK-WT MEFs were large cells with prominent stress fibers. Vinculin localized to the ends of the stress fibers. In contrast, FAK-KO MEFs were small round cells with large actin cables at the cell periphery. Vinculin plaques co-localized with actin bundles at cell margins. Bar $20 \mu \mathrm{m}$. C) Confluent endothelial cell monolayers: In WT endothelial monolayers vinculin was present at cell margins and localized to the ends of actin filaments. A greater number of vinculin plaques were evident in FAK-KD endothelial cells and actin filaments were disorganized compared to WT controls. Bar $20 \mu \mathrm{m}$. 


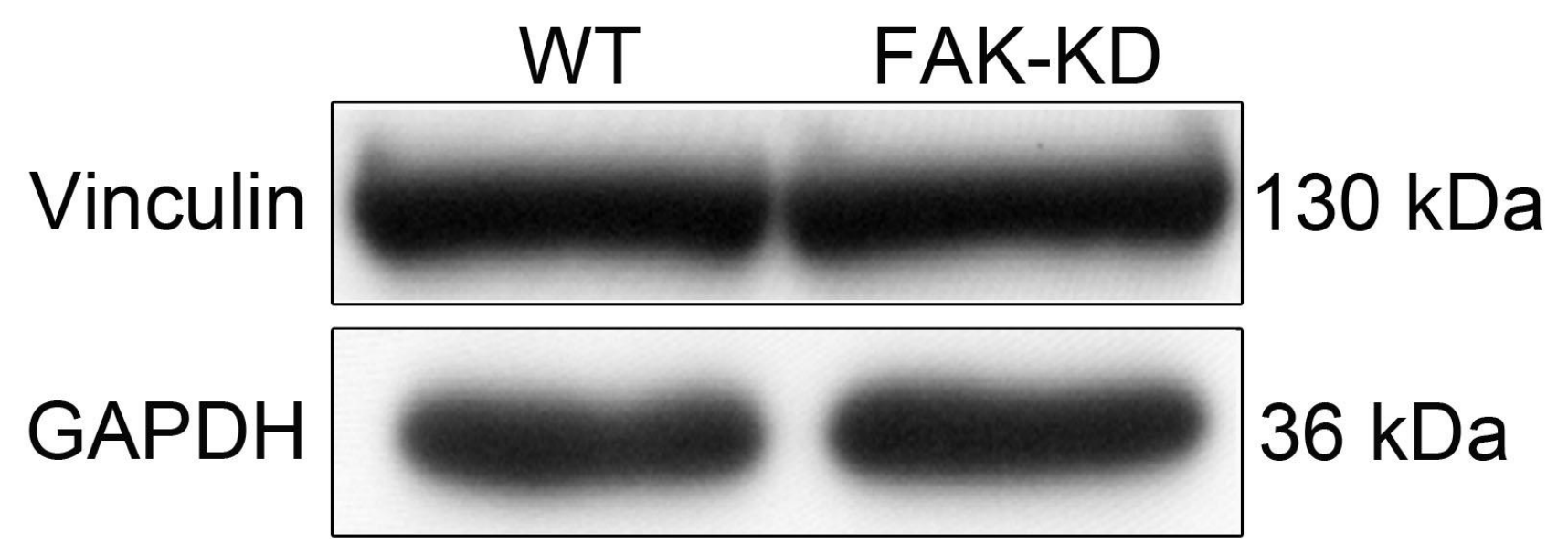

Figure 5 
Figure 5: Vinculin protein expression in FAK-KD and WT endothelial cells.

Endothelial cell extracts were analyzed by Western blots for vinculin protein levels. FAK knockdown did not alter vinculin protein levels in human pulmonary lung microvessel endothelial cells. GAPDH was used as a loading control. 


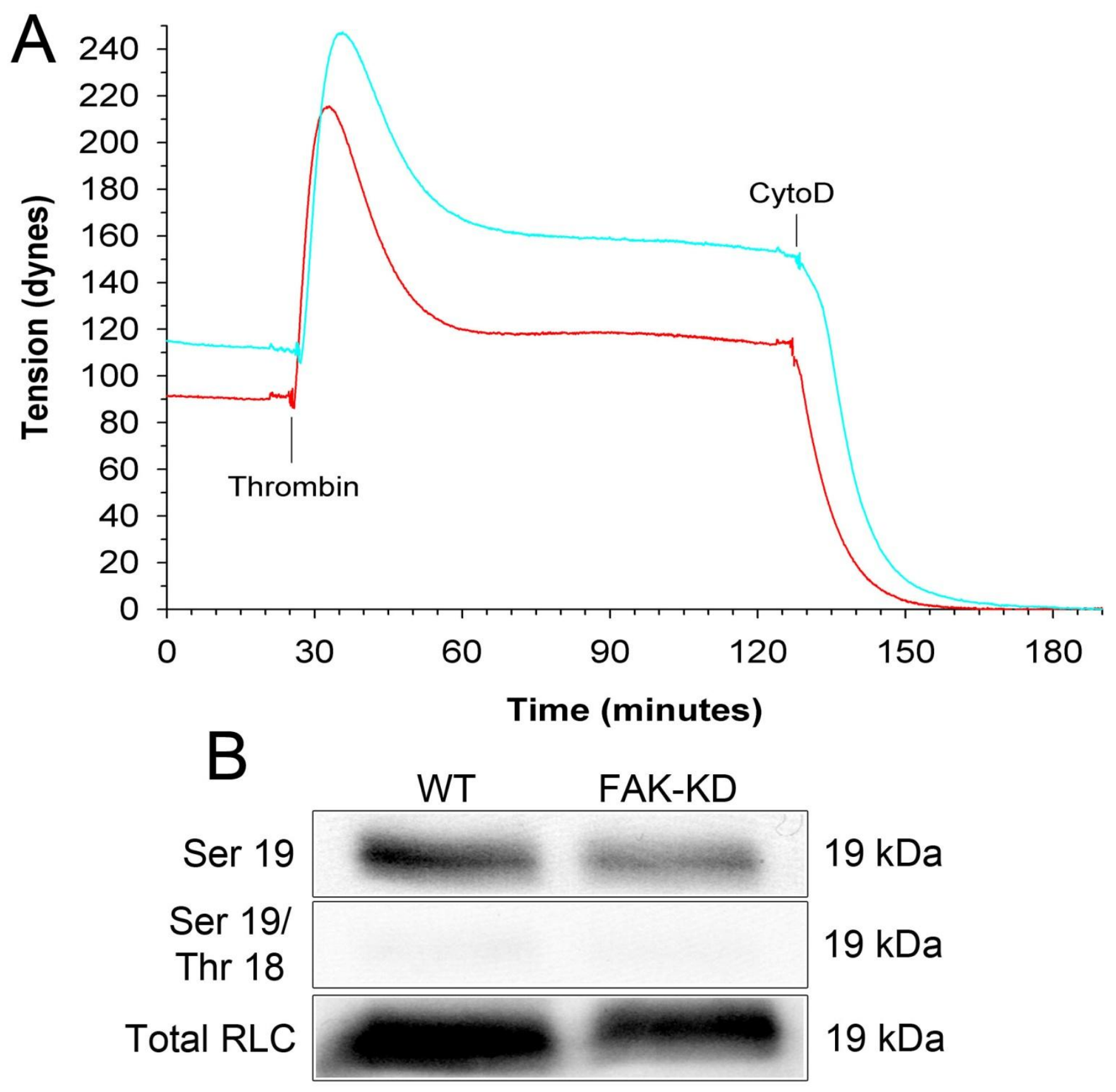

Figure 6 
Figure 6: Effect of FAK knockdown on endothelial cell isometric tension and myosin II phosphorylation.

A) Representative isometric tension tracing generated by WT (red) and FAK-KD (blue) monolayers exposed to $10 \mathrm{nM}$ thrombin. FAK knockdown monolayers developed a higher basal tension compared to controls. Loss of FAK had little effect on agonist induced tension generation. B) Effect of FAK knockdown on myosin II phosphorylation. WT and FAK-KD endothelial cell extracts were analyzed for monophosphorylated (Ser 19), diphosphorylated (Ser 19/Thr 18) myosin II RLC, and total RLC. Reduction in cellular levels of FAK did not alter basal myosin RLC phosphorylation. 


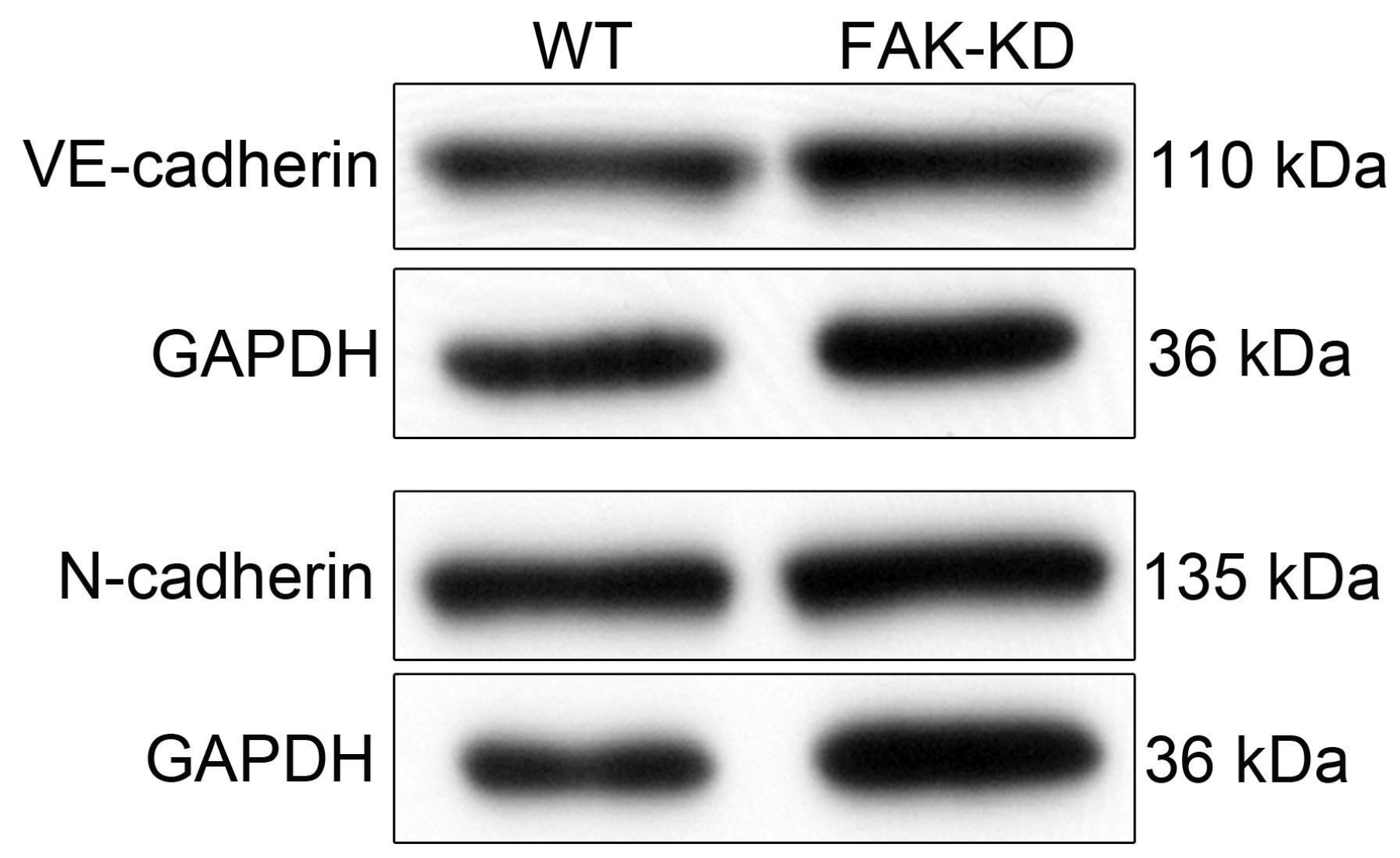

Figure 7 
Figure 7: VE- and N-cadherin protein expression in FAK-KD and WT endothelial cells. Endothelial cell extracts were analyzed by western blots for VE-and N-cadherin protein levels. FAK knockdown did not alter the level of either VE- or N-cadherin protein expression in human pulmonary lung microvessel endothelial cells. GAPDH was used as a loading control. 

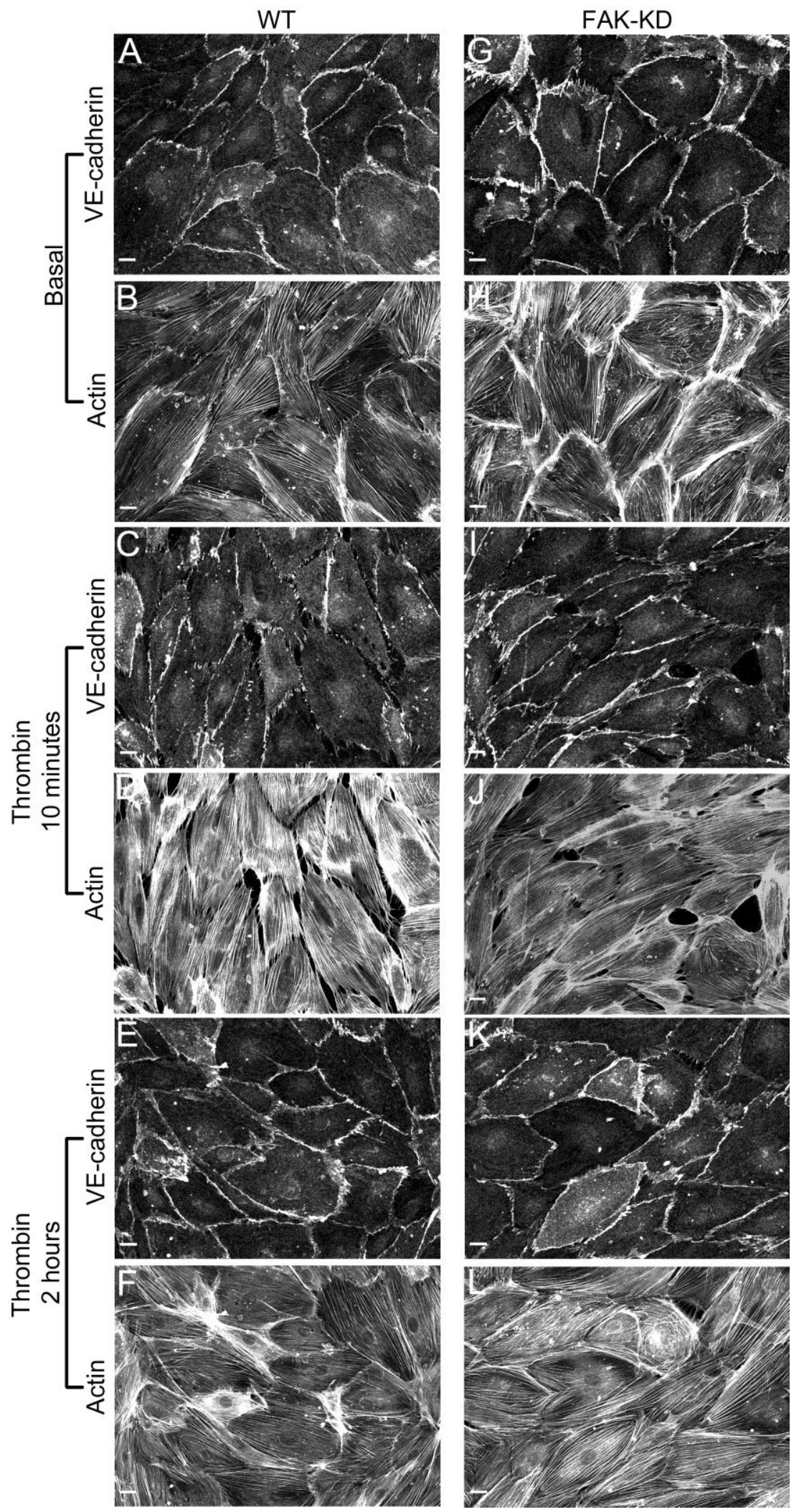

Figure 8 


\section{Figure 8: Localization of VE-cadherin and F-actin in WT and FAK-KD HLECs.}

Endothelial cells were seeded onto collagen-coated cultured dishes and fixed four days post confluence and stained for VE-cadherin (A,C,E,G,I,K) and F-actin (B,D,F,H,J,L) by rhodamine phalloidin binding as outlined in Materials and Methods. In control and FAK-KD unstimulated endothelial cell monolayers, VE-cadherin $(\mathbf{A}, \mathbf{G})$ was localized as a continuous rim of fluorescence at cell margins while actin $(\mathbf{B}, \mathbf{H})$ was bundled into prominent filaments running the length of cells. WT and FAK-KD monolayers were incubated with $10 \mathrm{nM}$ thrombin for $10 \mathrm{~min}$ and fixed for immuno-staining. Thrombin induced gap formation between cells with loss of VEcadherin staining $(\mathbf{C}, \mathbf{I})$ at the membranes which outline gaps. By two hours, gaps within the monolayers closed and VE-cadherin (E, K) was present at cell-cell contacts. Bar $20 \mu \mathrm{m}$. 


\title{
Chapter 3
}

\section{Focal adhesion kinase (FAK) is necessary for tension generation and organization of the collagen matrix}

\author{
Kimberly M. Arnold ${ }^{\mathrm{a}, \mathrm{d}}$, Zoe M. Goeckeler ${ }^{\mathrm{b}, \mathrm{d}}$, and Robert B. Wysolmerski ${ }^{\mathrm{b}, \mathrm{c}, \mathrm{d}, \uparrow}$ \\ Departments of Physiology and Pharmacology ${ }^{\mathrm{a}}$, Neurobiology and Anatomy ${ }^{\mathrm{b}}$, Mary Babb \\ Randolph Cancer Center ${ }^{\mathrm{c}}$, and Center for Cardiovascular and Respiratory Sciences ${ }^{\mathrm{d}}$, West \\ Virginia University School of Medicine, Morgantown, WV 26506
}

Running title: FAK is critical for tension generation

Corresponding Author ${ }^{\dagger}$ :

Robert B. Wysolmerski, Ph.D.

Department of Neurobiology and Anatomy

One Medical Center Drive

PO Box 9128

West Virginia University School of Medicine

Morgantown, WV 26506

Tel: 304-293-2213

Fax: 304-293-5513

Email: rywsolmerski@hsc.wvu.edu 


\begin{abstract}
Focal adhesion kinase (FAK) is an essential molecule within focal adhesions and has been proposed to be a key component in the transduction of cellular forces between cellular environments. Utilizing FAK knockout (FAK-KO) mouse embryonic fibroblasts (MEFs) embedded in three-dimensional (3-D) collagen gels, we found that loss of FAK decreased basal tension and produced minimal agonist induced force compared to controls without alterations in myosin II phosphorylation. Analysis of extracellular matrix structure showed controls generated an organized, dense collagen network whereas FAK-KO formed a loose matrix lacking a distinct organizational pattern. Re-expression of FAK in knockouts rescued force development and matrix organization. Inhibition of FAK kinase activity had little effect on basal or agonist induced force generation where as inhibition of FAK localization to focal adhesions reduced basal force and nearly abolished agonist induced force. Our findings indicate FAK is an important structural molecule within focal adhesions, facilitating transduction of cellular forces from inside to outside of the cell.
\end{abstract}




\section{List of Abbreviations:}

2-D - two-dimensional

3-D - three-dimensional

C4 - chloropyramine hydrochloride

ECM - extracellular matrix

FAK - focal adhesion kinase

FAK180/3 - FAK knockout fibroblasts expressing constitutively active FAK

FAK-dead - FAK knockout fibroblasts expressing kinase dead mutant of FAK

FAK-KO - focal adhesion kinase knockout mouse embryonic fibroblasts

FAK-WT - wild-type littermates of FAK-KO mouse embryonic fibroblasts

FRNK- FAK knockout fibroblasts expressing a truncated form of FAK

FRNK-C1034S - FAK knockout fibroblasts expressing FRNK targeting mutant

MEFs - mouse embryonic fibroblasts

p53-KO - p53 knockout mouse embryonic fibroblasts

Pyk2 - FAK-related proline-rich tyrosine kinase 2

RLC - nonmuscle myosin II regulatory light chain

SEM - scanning electron microscopy

SHG - second harmonic generation

WT-FAK - FAK knockout fibroblasts expressing full-length focal adhesion kinase 


\section{Introduction}

Originally discovered as a substrate for $\mathrm{v}-\mathrm{Src}$, focal adhesion kinase (FAK) is a cytoplasmic tyrosine kinase whose name is derived from its subcellular localization (Kanner et al., 1990; Schaller et al., 1992, Kornberg et al., 1991; Guan et al., 1991; Hanks et al., 1992) and has been found to be a key structural and functional protein involved in signaling pathways that regulate cell adhesion, migration, and survival (Ilic et al., 1995; Ilic et al., 1998; Zhao et al., 1998; Owen et al., 1999; Renshaw et al., 1999; Sieg et al., 1999; Sieg et al., 2000; Webb et al., 2002). Localized in multiprotein complexes called focal adhesions, FAK has been shown to be a prominent protein in adhesion structure and associated signaling pathways (Parsons, 1993; Schaller and Parsons, 1994; Richardson and Parsons, 1995). Focal adhesions allow the cell to interact with its external environment through transmembrane receptors called integrins, which bind to the extracellular matrix (ECM) proteins and create a physical linkage for the cell to its surroundings (Burridge et al., 1988; Jockusch et al., 1995; Liu et al., 2000). It also provides the mechanism necessary for the cell to respond to external cues (outside-in signaling) as well as influence its own surroundings (inside-out signaling) (Hynes, 2002). Internally, the integrinmediated cellular adhesions are linked to the contractile machinery (actin and myosin II) through the focal adhesions and connect the actin cytoskeleton with the ECM (Jockusch et al., 1995; Hynes, 2002; Zaidel-Bar and Geiger, 2010). These specialized sites of adhesion provide the structural link needed for the cell to generate tension and the disruption of these cell-matrix adhesions leads to the decline in cellular tension (Balaban et al., 2001; Riveline et al., 2001). However, how these adhesions are involved in the generation and transmission of force is not well understood. 
The generation of cell tension occurs when myosin II interacts with actin cytoskeletal filaments and is regulated primarily by the phosphorylation status of myosin II, which can occur through direct phosphorylation of myosin regulatory light chains (RLC) or inhibition of myosin phosphatase. Phosphorylation of myosin II through both calcium-dependent and -independent pathways regulates motor activity, myosin bipolar filament assembly, and stimulates actinactivated myosin II ATPase activity leading to changes in cell contractility (Scholey et al., 1980; Tan et al., 1992). Control of cell contraction occurs through myosin light chain kinase (MLCK), which becomes activated following the binding of calcium dependent calmodulin and directly phosphorylates myosin II (Scholey et al., 1980). Also, active Rho-kinase (ROCK), which is controlled by the small GTPase Rho, can inhibit the myosin II light chain phosphatase as well as directly phosphorylate myosin II in a calcium-independent manner suggesting there are alternative pathways for the regulation of myosin II and cell contraction (Amano et al., 1996; Kimura et al., 1996; Kureishi et al., 1997; Kawano et al., 1999; Totsukawa et al., 2000; Emmert et al., 2004.

A limited number of studies have investigated a role for FAK in cellular tension. Previous studies have shown that FAK is involved in the regulation of myosin II activation. In FAK null fibroblasts, the intrinsic activity of RhoA is increased and re-expression of FAK in these fibroblasts decreases RhoA activity and increases p190Rho-GTPase activating protein (p190RhoGAP) activity, implying FAK works, in part, through modulation of RhoA (Ren et al., 2000; Holinstat et al., 2006; Pirone et al., 2006). The impaired motility of FAK deficient fibroblasts can be rescued by pharmacological inhibition of ROCK (Chen et al., 2002). Recent indirect evidence has shown that the loss of FAK also results in an increase in ROCK activity. In addition to ROCK, MLCK activity is elevated in the absence of FAK suggesting that 
contractile activity should be increased (Chen et al., 2002; Webb et al., 2004). Abnormal regulation of Rho and the kinases that activate myosin II and regulate cytoskeletal dynamics implicates a role for FAK in the signaling pathways regulating cell tension. However, when FAK is knocked down in smooth muscle cells, there is a decrease in stimulated force production accompanied by a decrease in intracellular free calcium and myosin activation (Tang and Gunst, 2001). In a study conducted in our laboratory, we found that FAK knockdown in pulmonary endothelial cells resulted in an increase in basal cell tension without alterations in myosin II phosphorylation (Arnold et al., 2013). In addition, a limited number of studies using traction force and microneedle analysis suggest the loss of FAK alters cellular rigidity in nonmuscle cells (Pirone et al., 2006; Klemm et al., 2009; Fabry et al., 2011). Furthermore, studies have shown that extracellular stresses can activate FAK and result in an increase in tension (Li et al., 1997; Sawada and Sheetz, 2002) indicating discrepancies in FAK's involvement in tension generation. Also, there has been no direct measurement of how the loss of FAK affects overall cellular tension. Therefore, we wanted to determine if FAK is critical for nonmuscle cellular tension generation.

Much of the knowledge about FAK's role in cellular functions is derived primarily from single-cell in vitro experiments on fibronectin coated glass coverslips with a limited number of studies performed on collagen-coated dishes. However, physiologically, cells behave in a cohesive unit. Also, the focal adhesion molecular composition and structure following attachment to a two-dimensional (2-D) substrate differs in three-dimensional (3-D) matrix adhesions (Cukierman et al., 2001). Changes in the molecular composition of the focal adhesions could alter cellular behavior in 3-D. Therefore, we wanted to determine the involvement of FAK in tension generation in cells embedded within a 3-D collagen matrix. 
To investigate the role of FAK in tension generation, we utilized mouse embryonic fibroblasts (MEFs) isolated from FAK knockout (FAK-KO) mice and embedded the cells within a 3-D collagen matrix. Unexpectedly, fibroblasts devoid of FAK had low basal force and were unable to produce tension in response to an agonist. Myosin II activation and actin stress fiber formation occurred following treatment of FAK-KO MEFs with an agonist indicating the contractile machinery was intact. However, altered cell morphology and lack of distinct focal adhesion formation in 3-D suggested impairment in the focal adhesion linkage to the external cellular surroundings. Imaging of the collagen matrix revealed a lack of collagen organization by the FAK-KO MEFs indicating a loss of functional interaction with the collagen. Inhibition of FAK kinase activity did not affect force generation; however, disruption of FAK localization within the focal adhesion reduced basal force and blunted agonist induced force generation similar to that observed by the FAK-KO MEFs. Thus, FAK is a critical protein in providing the scaffolding necessary for internal cellular tension to be transmitted to the external matrix resulting in cellular interaction and organization of the matrix in order for cell contraction to occur.

\section{Materials and Methods}

\section{Cell Culture}

Mouse embryonic FAK knockout (FAK-KO), wild-type littermates (FAK-WT), and p53 knockout (p53-KO) fibroblasts were purchased from ATCC (Manassas, VA) and maintained in Dulbecco's modified Eagle's medium (DMEM) supplemented with 10\% fetal calf serum (FCS) (Sigma-Aldrich, St. Louis, MO). FAK-WT and FAK-KO MEFs are of mesenchymal origin, since they express vimentin and lack E-cadherin. The p53-null mutation was introduced to 
enable propagation of primary FAK-KO cells (Tsukada et al., 1993; Ilic et al., 1995; Ilic et al., 1998). Control fibroblasts were isolated from mouse embryos as described previously (Connor, 2001) and maintained in DMEM supplemented with $10 \%$ FCS. All cells were cultured at $37^{\circ} \mathrm{C}$, $5 \% \mathrm{CO}_{2}$.

\section{Rat Tail Type I Collagen Isolation}

Four fresh rat tails were washed with water, soaked in $70 \%$ ethanol, and rinsed with phosphatebuffered saline (PBS) prior to skinning. The tip of the tail was cut off and an incision was made from the base to the tip allowing the skin to be easily peeled off. A cut was made through each collagen bundle at the tip and base of the tail in order to pull the collagen fibrils from the tail. Pulled collagen fibrils were placed on ice in a $100 \mathrm{~mm}$ dish containing PBS until approximately 5 grams of pulled collagen fibrils (wet weight) had been obtained. All fibrils were then placed on ice in a $100 \mathrm{~mm}$ dish containing $70 \%$ ethanol for 1 hour. Collagen fibrils were rinsed twice in PBS and once in water before placing in a beaker containing $20 \mathrm{mM}$ acetic acid. To extract collagen, solution was stirred very slowly at $4^{\circ} \mathrm{C}$ for 48 hours. After 48 hours, the solution was spun at $16,000 \mathrm{~g}$ for 90 minutes at $4^{\circ} \mathrm{C}$ to remove any fibrils that were not extracted. Collagen supernatant was stored at $4^{\circ} \mathrm{C}$. Collagen concentration was determined by running serial dilutions of a commercially bought collagen with known concentration on a $10 \%$ SDS gel to generate a standard curve. Serial dilutions of extracted collagen were run on a separate gel. Both gels were stained with Coomassie Blue and an Odyssey infrared imaging system (LI-COR Biosciences, Lincoln, NE) was used to scan gels and quantitate the bands to determine the concentration of the extracted collagen. 


\section{Isometric Tension Measurements}

Isometric tension measurements were performed as previously described (Kolodney and Wysolmerski, 1992; Goeckeler and Wysolmerski, 1995, Goeckeler et al., 2008). Briefly, collagen gels were made by suspending $1 \times 10^{6}$ cells $/ \mathrm{ml}$ in a collagen solution containing $1 \mathrm{mg} / \mathrm{ml}$ rat tail type I collagen and seeded into a cylindrical Teflon mold which contains a central post. Cells cannot bind to the Teflon and therefore only interact with themselves or the collagen fibers. Cells bind to the collagen and constrict and organize the fibers around the mold's central post to form a ring of cells embedded within collagen which will be referred to as collagen gels. Cells were grown for 4 days at $37^{\circ} \mathrm{C}, 5 \% \mathrm{CO}_{2}$. After 4 days, collagen gels were gently removed from the molds and hung from force transducers to monitor tension. After establishing a stable basal tension, cells were stimulated with $1 \mu \mathrm{M}$ lysophosphatidic acid (LPA) and actin filament polymerization was inhibited with cytochalasian D (CytoD). Following experiments, collagen gels were snap frozen for measurement of DNA content.

\section{DNA Quantitation and Staining}

In order to normalize force measurements, the number of cells within a collagen gel was determined by quantitation of DNA content using Hoechst 33258 dye as described previously (Emmert et al., 2004; Goeckeler et al., 2008). Salmon testes DNA was used to generate a standard curve. Collagen gels from representative force experiments were probe sonicated in $750 \mu 10.1 \%$ SDS/PBS until a homogenous solution was generated. Unknown samples were diluted 1:50 and 1:100 in PBS and $100 \mu$ of sample was loaded into the appropriate well of a microtiter plate. $200 \mu \mathrm{l}$ of $0.135 \mu \mathrm{g} / \mathrm{ml}$ stock Hoechst 33258 dye was added to each well for a final concentration of $0.09 \mu \mathrm{g} / \mathrm{ml}$. Fluorescence was measured using a Modulus Microplate 
Multimode Reader (Turner BioSystems, Inc, Sunnyvale, CA). DNA concentration within each collagen gel was calculated using the slope of the standard curve and the raw intensity reading of the unknown.

To determine dispersion of cells within the collagen gels, FAK-KO collagen gels were fixed overnight in stabilization buffer $\left(127 \mathrm{mM} \mathrm{NaCl}, 5 \mathrm{mM} \mathrm{KCl}, 1.1 \mathrm{mM} \mathrm{NaH}_{2} \mathrm{PO}_{4}\right.$ monobasic, $0.4 \mathrm{mM} \mathrm{KH}_{2} \mathrm{PO}_{4}, 2 \mathrm{mM} \mathrm{MgCl}$, $5.5 \mathrm{mM}$ glucose, $1 \mathrm{mM}$ EGTA, $20 \mathrm{mM}$ Pipes) containing 2\% glutaraldehyde on day 1,2,3, and 4 post-seeding. Two small pieces of the collagen gel were cut and cells were permeabilized in stabilization buffer containing $0.5 \%$ Triton X-100 and $0.6 \%$ deoxycholate (DOC) for 3 hours and incubated with $4 \mu 1$ rhodamine phalloidin (Sigma-Aldrich, St. Louis, MO; Cat.\#P1951) overnight at room temperature. The following day, collagen gels were washed extensively with PBS and incubated with $1 \mu \mathrm{g} / \mathrm{ml}$ Hoechst 33258 dye for 20 minutes to stain cell nuclei. To image the cells, stained gel pieces were coverslipped with $90 \%$ glycerol/10\% PBS containing 0.1 M n-propyl gallate (Giloh and Sedat, 1982). Z-stack images of the collagen gel were generated by taking $1.5 \mu \mathrm{m}$ steps through the total depth of the gel using two-photon fluorescence microscopy (Yang et al., 2011, 2013).

\section{Myosin Phosphorylation}

The extent of myosin II RLC phosphorylation was determined 4 days after casting cells in collagen gels. After 4 days, gels were stimulated with 2 units $/ \mathrm{ml}$ thrombin for 2.5 minutes while still in the mold. Collagen gels were removed from the mold, spun down for 2 minutes at 10,000 rpm in $1.5 \mathrm{ml}$ microfuge tube, supernatant was removed, and collagen gel was snap frozen for analysis. Thawed collagen gels were broken up with a small pestle, vortexed, and sonitcated in ice cold lysis buffer (600 mM sodium chloride). Samples were placed on ice for 15 minutes and 
then spun down at $10,000 \mathrm{rpm}$ for 10 minutes at $4^{\circ} \mathrm{C}$. Supernatant was removed and extract was combined with $4 \mathrm{x}$ SDS sample buffer. Samples were sonicated and heated to $100^{\circ} \mathrm{C}$ for 10 minutes and separated on $10 \%$ SDS gels. Proteins were transferred to nitrocellulose membranes and blots were air-dried overnight. The following day, membranes were re-hydrated in 1x Trisbuffered saline (TBS), fixed with $0.1 \%$ glutaraldehyde/PBS, reduced in $50 \mathrm{mM}$ sodium borohydride, and blocked in 5\% milk/1x TBS/0.1\% Tween-20 prior to the addition of primary antibodies. Blots were incubated in either a 1:1,000 dilution of anti-phospho-Ser19 myosin II regulatory light chain (RLC) antibody (Cell Signaling Technology, Danvers, MA; Cat.\#3671) or affinity-purified anti-myosin RLC antibody raised against recombinant nonmuscle myosin II RLC as outlined previously (Chew et al., 1998) overnight at $4^{\circ} \mathrm{C}$. To visualize proteins, Western blots were incubated in a 1:8,000 dilution of HRP-conjugated goat anti-rabbit (Bio-Rad Laboratories, Hercules, CA; Cat.\#1706515) for 1 hour at room temperature, washed with $1 \mathrm{x}$ TBS/0.1\% Tween-20, and incubated in ECL reagents (GE Healthcare, Piscataway, NJ). Blots were exposed on X-ray films to detect signal emitted from protein bands. Levels of phosphorylated myosin RLC were compared to total myosin RLC protein levels.

\section{Gel compression studies}

Cells embedded within collagen gels were incubated in the Teflon molds with $10 \mu \mathrm{M}$ CellTracker Red CMPTX (Invitrogen Life Technologies, Grand Island, NY; Cat.\#C34552) 4 days post seeding for 30 minutes at $37^{\circ} \mathrm{C}$ and then washed with media for 30 minutes at $37^{\circ} \mathrm{C}$. Following incubation, collagen gels were removed from the molds and fixed in stabilization buffer containing 3\% formaldehyde overnight at room temperature. To determine the depth of an uncompressed collagen gel devoid of cells, $10 \mu \mathrm{l}$ Fluoresbrite multifluorescent $1 \mu \mathrm{m}$ 
microspheres (Polysciences, Inc, Warrington, PA; Cat.\#24062) were added to $1 \mathrm{ml}$ of the collagen solution prior to casting in Teflon molds. After 4 days, the collagen gels devoid of cells were removed and fixed overnight. Collagen gels were washed with PBS and coverslipped with 90\% glycerol/10\% PBS containing 0.1 M n-propyl gallate (Giloh and Sedat, 1982). Two small pieces from each collagen gel were cut and four different areas from each piece were measured. Four different molds were used per cell type. Four images were taken in varying locations throughout each collagen gel piece. The total depth of the collagen gel was measured using twophoton fluorescent microscopy (Yang et al., 2011, 2013) by imaging the fluorescence to determine the top and bottom of the gel.

\section{Two-Dimensional (2-D) Immunofluorescence}

Immunostaining procedures followed the protocols described in Goeckeler and Wysolmerski (1995). $3 \times 10^{4}$ cells were seeded onto $35-\mathrm{mm}$ collagen-coated dishes ( $25 \mu \mathrm{g} / \mathrm{ml}$ type I collagen) and incubated overnight at $37^{\circ} \mathrm{C}, 5 \% \mathrm{CO}_{2}$. Cells were fixed and permeabilized in $37^{\circ} \mathrm{C}$ stabilization buffer containing $2 \%$ formaldehyde, $0.2 \%$ Triton X-100, and $0.5 \%$ DOC overnight at room temperature. Cultures were permeabilized in stabilization buffer containing $0.5 \%$ Triton $\mathrm{X}-100,0.6 \%$ DOC for two 10 minute incubations, gently washed with PBS, and incubated in 10 $\mathrm{mM}$ sodium borohydride for 2 minutes to reduce free aldehydes. To reduce non-specific binding, cells were incubated in blocking buffer (PBS containing $0.4 \%$ bovine serum albumin, $0.05 \%$ fish gelatin, $10 \mu \mathrm{g} / \mathrm{ml}$ goat $\mathrm{IgG}$ ) for 1 hour prior to incubation with primary antibodies. For focal adhesion staining, cells were incubated with a 1:1,500 dilution of monoclonal mouse anti-vinculin antibody (Sigma-Aldrich, St. Louis, MO; Cat.\#V9131) for 3 hours and then a 1:1,000 dilution of Alexa goat anti-mouse $647 \mathrm{~nm}$ antibody (Invitrogen Life Technologies, 
Grand Island, NY; Cat.\#A21237) for 1 hour. To visualize actin filaments, cells were incubated with $2 \mu$ l rhodamine phalloidin/35-mm dish (Sigma-Aldrich, St. Louis, MO; Cat.\#P1951) for 1 hour. Cells were washed with PBS and then coverslipped with $90 \%$ glycerol/10\% PBS containing 0.1 M n-propyl gallate (Giloh and Sedat, 1982). Cells were examined using a Zeiss LSM 510 confocal microscope (63x oil objective).

\section{Focal Adhesion Immunostaining in Three-Dimensional (3-D) Collagen Gels}

Differences in 3-D cell morphology and focal adhesions were assessed by generating collagen gels as described previously. After 4 days, collagen gels were removed from the molds, washed in PBS, and fixed and permeabilized in stabilization buffer containing $2 \%$ formaldehyde, $0.2 \%$ Triton X-100, and 0.5\% DOC overnight. A small piece from each collagen gel was randomly cut for immunostaining. For immunofluorescence, cells were permeabilized for 3 hours in $2 \mathrm{x}$ stabilization buffer containing $0.5 \%$ Triton X-100 and 0.6\% DOC followed by extensive washing with PBS. Cells were incubated with 1:500 dilution of monoclonal mouse anti-vinculin antibody and $4 \mu \mathrm{l}$ rhodamine phalloidin/piece of mold (Sigma-Aldrich, St. Louis, MO; Cat.\#V9131 and Cat\#.P1951, respectively) overnight at room temperature. The following day, cells were incubated with a 1:1,000 dilution of Alexa goat anti-mouse $488 \mathrm{~nm}$ antibody (Invitrogen Life Technologies, Grand Island, NY; Cat.\#A11017) for 3 hours. Collagen gels were washed well with PBS and coverslipped with $90 \%$ glycerol/10\% PBS containing $0.1 \mathrm{M} n$-propyl gallate (Giloh and Sedat, 1982). Z-stack images of the collagen gel were generated by taking $1.5 \mu \mathrm{m}$ steps through the total depth of the gel using two-photon fluorescent microscopy (Yang et al., 2011, 2013). 


\section{Western Blot Analysis}

Cells cultured in 2-D were scraped up into $100^{\circ} \mathrm{C} 1.5 \mathrm{x}$ SDS sample buffer containing $10 \mu \mathrm{g} / \mathrm{ml}$ DNase I. For 3-D samples, cell-collagen gels were probe sonicated in $500 \mu \mathrm{l} 0.1 \% \mathrm{SDS} / \mathrm{PBS}$ to break up the collagen. $30 \mu \mathrm{l}$ of cell-collagen suspension was combined with $3 \mathrm{x}$ SDS sample buffer containing $10 \mu \mathrm{g} / \mathrm{ml}$ DNase I for protein content analysis. All samples were sonicated and heated to $100^{\circ} \mathrm{C}$ for 10 minutes and separated on $10 \%$ SDS gels. Proteins were transferred to PVDF membranes and blocked in $5 \%$ milk prior to the addition of primary antibodies. Blots were incubated with the following antibodies: 1:12,000 dilution of mouse monoclonal antiGAPDH (Fitzgerald Industries International, Acton, MA; Cat.\#10R-G109A); 1:1,000 dilution of rabbit polyclonal anti-FAK (C-20), 1:800 dilution of rabbit polyclonal anti-Pyk2 (Santa Cruz Biotechnology Inc., Dallas, TX; Cat.\#SC-558 and Cat.\#SC-9019, respectively); 1:1,000 dilution of rabbit polyclonal anti-FAK pY397 (Invitrogen Life Technologies, Grand Island, NY; Cat.\#44$624 \mathrm{G}) ; 1: 1,000$ dilution of rabbit polyclonal anti- $\alpha_{1}$ integrin (Abcam, Cambridge, MA; Cat.\#ab106267); 1:2,500 dilution of mouse monoclonal anti-p53 (1C12) and 1:1,000 dilution of rabbit polyclonal anti- $\alpha_{5}$ integrin, anti- $\alpha_{V}$ integrin, anti- $\beta_{1}$ integrin, or anti- $\beta_{3}$ integrin (Cell Signaling Technology, Danvers, MA; Cat.\#2524, Cat.\#4705, Cat.\#4711, Cat.\# 4706, and Cat.\#4702, respectively) overnight at $4^{\circ} \mathrm{C}$. For visualization of proteins, Western blots were incubated in a 1:8,000 dilution of either HRP-conjugated goat anti-rabbit or goat anti-mouse (Bio-Rad Laboratories, Hercules, CA; Cat.\#1706515 and Cat.\#1706516, respectively) antibodies for 1 hour at room temperature, washed with $1 \mathrm{x}$ TBS/0.1\% Tween-20, and incubated in ECL reagents (GE Healthcare, Piscataway, NJ). Blots were exposed on X-ray films to detect signal emitted from protein bands. Protein levels were quantitated with laser densitometry. GAPDH was used as loading control in all Western blots. 


\section{Second Harmonic Generation (SHG) and Pixel Intensity Quantitation}

Collagen gels were removed and fixed in stabilization buffer containing $2 \%$ formaladehyde overnight. The collagen gel was cut and laid flat and anchored in a 35-mm dish filled with PBS for imaging. Second harmonic (SHG) was generated as outlined by Yang et al. (2011, 2013). Pixel intensity in six, random regions of interest was determined for each image utilizing the image-processing package Fiji, an Open Source image processing package based on ImageJ. Pixel intensity was quantified by subtracting the background from the total pixel intensity of each region of interest. Data was presented as average pixel intensity per cell type. Error bars represent standard error of the mean. 10 separate images from each cell type were analyzed.

\section{Scanning Electron Microscopy}

Collagen gels were washed in $37^{\circ} \mathrm{C}$ PBS prior to immersion fixation in $1 \%$ formaldehyde, $2.5 \%$ glutaraldehyde in $0.15 \mathrm{M}$ sodium cacodylate buffer $\mathrm{pH} 7.2$ for 48 hours at $4^{\circ} \mathrm{C}$. Samples were washed in sodium cacodylate buffer and post-fixed in $1 \%$ osmium tetroxide in the cacodylate buffer for 30 minutes. Samples were rapidly dehydrated in graded steps of acetone (25\%-100\%) and critically point dried using a Tousimis 815 a Critical Point Dryer. Samples were mounted onto aluminum stubs and coated with a $40 \mathrm{~nm}$-thick layer of platinum using a Temescal BJD 200 E-Beam Evaporator. Samples were examined with a JEOL JSM-7600-F scanning electron microscope.

\section{Rescue of FAK knockout}

Re-expression of FAK in FAK-KO fibroblasts was achieved by using a tetracycline inducible lentiviral vector encoding either a wild-type form of FAK (WT-FAK) or a constitutively active 
form of FAK (FAK180/3) (Lietha et al., 2007). Constructs were generously provided by Dr. Michael Schaller. Lentivirus was generated by transfecting subconfluent $293 \mathrm{~T}$ cells with the lentiviral vector and the $2^{\text {nd }}$ generation packaging system, psPAX2 and pCMV-VSVG (3:2:1 ratio of DNA concentration, respectively) and the transfecting agent, TurboFect (Thermo Scientific, Waltham, MA; Cat.\#R0531). Supernatants containing viral particles were collected every 12 hours for 96 hours and stored at $4^{\circ} \mathrm{C}$ during collection. Viral supernatants were combined and centrifuged at 2,000 rpm for 10 minutes at $4^{\circ} \mathrm{C}$, filtered through a $0.22 \mu \mathrm{m}$ low protein binding syringe filter, and frozen at $-80^{\circ} \mathrm{C}$. To generate $\mathrm{FAK}$ re-expressing fibroblasts, FAK-KO MEFs were seeded at a density of $5 \times 10^{5}$ cells/T-25 flask and allowed to adhere and spread 24 hours prior to addition of WT-FAK or FAK180/3 lentivirus. After 72 hours, virus was removed and cells were placed in normal growth media to recover. In order to select for FAK re-expressing cells, infected cells were seeded at a density of $5 \times 10^{5}$ cells/T-25 flask in the presence of $1 \mu \mathrm{g} / \mathrm{ml}$ puromycin. The lentiviral vector carries a resistance gene for puromycin and therefore only infected cells will be able to survive the presence of puromycin. Since the lentiviral vector was also tetracycline inducible, WT-FAK or FAK180/3 expression was induced by addition of $1 \mu \mathrm{g} / \mathrm{ml}$ doxycycline. Stably FAK re-expressing cell lines were maintained in full media containing $1 \mu \mathrm{g} / \mathrm{ml}$ puromycin and $1 \mu \mathrm{g} / \mathrm{ml}$ doxycycline. FAK protein levels were measured by Western blot to ensure re-expression of FAK.

\section{FAK Inhibitors and Mutant Constructs}

For inhibitor studies, the small molecule inhibitor, PF-573,228 (Slack-Davis et al. 2007) was used to inhibit FAK kinase activity and the small molecule chloropyramine hydrochloride (C4) (Kurenova et al., 2009) was utilized to disrupt FAK targeting to focal adhesions. FAK-WT and 
FAK-KO collagen gels were made and hung on force transducers as described previously. $1 \mu \mathrm{M}$ of PF-573,228 was added to the bath after establishment of stable baseline tension. After 15 minutes of inhibitor treatment, cells were stimulated with $1 \mu \mathrm{M}$ LPA to determine the effect of FAK kinase inhibition on agonist induced tension. Collagen gels were snap frozen for measurement of DNA content following the experiment. For the $\mathrm{C} 4$ inhibitor experiments, $5 \mu \mathrm{M}$ C4 was added to the media 24 hours post seeding FAK-WT cell-collagen solution into Teflon molds and replenished with new inhibitor every 24 hours. After 4 days, collagen gels were removed from molds and hung from force transducers as described previously and allowed to generate a stable basal tension. After basal tension was achieved, $5 \mu \mathrm{M}$ of $\mathrm{C} 4$ was added for 1 hour prior to stimulation with $1 \mu \mathrm{M}$ LPA. Collagen gels were snap frozen for measurement of DNA content following experiment. To inhibit the activity of the Src-family of kinases, FAKWT collagen gels were made and hung from force transducers as described previously. Following establishment of basal tension, either $1 \mu \mathrm{M}$ of PP2 (Enzo Life Sciences, Farmingdale, NY; Cat.\#BML-EI297) or $1 \mu$ M SU6656 (Calbiochem, San Diego, CA; Cat.\#572635) was added to the bath for 15 minutes to inhibit the activity of the Src-family of kinases prior to stimulation with $1 \mu$ M LPA.

To determine if FAK kinase activity was necessary for tension generation, FAK-KO MEFs were infected with an adenovirus encoding a kinase dead form of FAK (FAK-dead) which was generously provided by Dr. Kevin Pumiglia. FAK-KO MEFs were seeded at a density of $3.5 \times 10^{5}$ cells $/ 35 \mathrm{~mm}$ dish 24 hours prior to infection. Cells were infected with $5 \mu \mathrm{l}$ virus on day 1 and 2. On day 3, the virus was removed and cells were placed in normal growth media to recover. On the fourth day post-seeding, cells were trypsinized, counted, and $1 \times 10^{6}$ cells $/ \mathrm{ml}$ were resuspended in $1 \mathrm{mg} / \mathrm{ml}$ collagen solution for construction of collagen gels. Isometric 
tension measurements were performed as described previously. Following tension measurements, collagen gels were snap frozen for determination of infection efficiency. FAK protein levels were measured by Western blot to ensure expression of kinase dead FAK.

To ensure FAK localization to focal adhesions was critical for tension generation, collagen gels were generated as described previously. Following collagen polymerization, cells were infected while in the molds with an adenovirus expressing a naturally occurring variant of FAK called FRNK (ㅌK-related non-kinase) (Schaller et al., 1993; Richardson and Parsons, 1996; Nolan et al., 1999) or a mutant form of FRNK (FRNK-C1034S) (Bryant et al., 2006) which has residue 1034 in the FAT domain mutated, disrupting paxillin binding and localization within focal adhesions (Sieg et al., 1999, Tomar et al., 2009) overnight in DMEM+1\% FCS. Constructs were generously provided by Dr. Kevin Pumiglia. An MOI of 50 was used for the FRNK adenovirus and $5 \mu \mathrm{l}$ of FRNK-C1034S virus was utilized for infection. The next day, molds were fed with full media and incubated for 4 days total prior to performing isometric tension measurements. Following tension measurements, collagen gels were snap frozen for determination of infection efficiency. FRNK protein levels were measured by Western blot to ensure expression of FRNK.

\section{Results}

FAK is critical for basal and agonist-induced force generation

In order to determine the effect loss of FAK has on basal and agonist stimulated tension, FAK knockout (FAK-KO) mouse embryonic fibroblasts (MEFs) and control fibroblasts from littermates (FAK-WT) were utilized. Cells were seeded into three-dimensional (3-D) collagen gels as described in METHODS. Both cell lines established a stable basal tension; however 
FAK-KO had approximately 40\% reduction in basal tension compared to FAK-WT (Figure 1A). After stable basal tension was established, cells were stimulated with the addition of $1 \mu \mathrm{M}$ lysophosphatidic acid (LPA). LPA treatment rapidly induced a 70 dyne increase in FAK-WT tension and the establishment of a new baseline tension. In contrast, LPA treatment only induced a 5 dyne increase in FAK-KO tension, which slowly returned to baseline (Figure 1A). Tension generated by FAK-WT and FAK-KO in response to LPA was abolished by application of $1 \mu \mathrm{M}$ cytochalasin $\mathrm{D}(\mathrm{CytoD})$ (Figure 1A).

FAK-KO MEFs were isolated from mice that were deficient in both FAK and p53 and therefore control and p53 knockout (p53-KO) MEFs were utilized in a limited number of experiments for comparison. Analysis of cell extracts revealed a complete loss of FAK and p53 in FAK-KO MEFs, however the loss of FAK caused an elevation in a closely related protein, FAK-related protein rich kinase 2 (Pyk2) as previously reported (Lim et al., 2008; Weis et al., 2008) (Supplementary Figure 1B and 1C). FAK-WT and p53-KO MEFs were completely devoid of p53 protein however only p53-KO MEFs had an elevation in Pyk2 protein levels (Supplementary Figure 1B and 1C). FAK protein levels were unaltered in control, FAK-WT, and p53-KO MEFs (Supplementary Figure 1A).

To ensure the effect on MEF tension was due to the loss of FAK and not p53, isometric tension of p53-KO MEFs was measured. Control and p53-KO MEFs produced a similar basal tension, which was approximately 10 dynes lower than FAK-WT; FAK-KO MEFs had a 50\% reduction in basal tension compared to FAK-WT (Figure 1B). Control, FAK-WT, and p53-KO MEFs responded to $1 \mu \mathrm{M}$ LPA stimulation resulting in an 80 dyne, 110 dyne, and 50 dyne increase in tension, respectively, where as FAK-KO only produced a 5 dyne increase in tension 
following LPA stimulation (Figure 1B). Tension produced by all cell types was abolished by application of $1 \mu \mathrm{M}$ CytoD (Figure 1B).

Myosin II activation through phosphorylation of its regulatory light chain (RLC) is necessary for bipolar myosin filament formation and interaction of myosin with actin filaments thus allowing the generation of cellular force. Low levels or the inability to phosphorylate myosin could attribute to the reduction in FAK-KO MEF tension following agonist stimulation. To assess if myosin activation was altered in FAK-KO MEFs, levels of myosin II RLC phosphorylation were analyzed. Comparable low levels of phosphorylated myosin II RLC were detected under basal conditions and, following thrombin stimulation, a similar increase in myosin II RLC phosphorylation was observed in FAK-KO and FAK-WT MEFs indicating that myosin activation was unaltered in FAK-KO MEFs (Figure 1C). Thus, FAK is critical for basal and agonist induced tension generation, however does not regulate myosin activation.

\section{Loss of FAK alters cell morphology and focal adhesion formation in 2-D and 3-D}

Modifications in cell morphology and actin structure and disruption of focal adhesion integrity through the loss of FAK could affect the mechanical linkage of the cell to the extracellular matrix and directly alter cell tension generation. Therefore, we wanted to determine how the loss of FAK affected cell morphology and focal adhesion distribution in fibroblasts. Since a majority of studies investigating FAK's role in tension generation has been done on cells seeded in two-dimensional (2-D) culture, we first wanted to investigate how the loss of FAK affected fibroblast cell morphology and focal adhesion formation. In 2-D culture, control and FAK-WT MEFs exhibited a spread, fibroblast morphology with a number of cellular protrusions and actin filaments extending throughout the central part of the cell (Figure 2A). In contrast, 
FAK-KO MEFs were smaller and more rounded with no cellular protrusions. There was an abundance of actin filaments throughout the FAK-KO MEFs with an increase in cortical actin around the cell periphery (Figure 2A). In comparison, p53-KO MEFs were also round, nonspread cells with a thick cortical actin ring and short central actin filaments (Figure 2A).

Indirect immunofluorescent labeling of vinculin, a major focal adhesion protein, was performed to visualize focal adhesion distribution in MEFs under preconfluent 2-D conditions. Control and FAK-WT MEFs had elongated adhesions that were located throughout the entire cell but predominated around the periphery (Figure 2A). In comparison, p53-KO MEFs had smaller plaques with an increased number of adhesions centrally in the cell compared to controls (Figure 2A). FAK-KO MEFs had smaller, thicker focal adhesions with an increase in plaque formation throughout the entire cell (Figure 2A). Similar alterations in focal adhesion distribution were observed when cells were stained with paxillin (data not shown). Therefore, loss of FAK altered both cell morphology and focal adhesion distribution in 2-D.

We found that cell morphology was altered with the loss of FAK in 2-D culture, however force experiments were conducted on cells that were embedded in a 3-D collagen matrix, and therefore, may exhibit different behaviors than cells cultured on dishes. Thus, we wanted to investigate how cell morphology changed when cells were grown in the collagen gels. In order to investigate the cell morphology in 3-D, FAK-WT and FAK-KO MEFs embedded in the collagen gels were stained with rhodamine phalloidin to visualize actin. The morphology between the two cell types is distinct. FAK-WT MEFs were spread throughout the collagen gel and were interconnected, forming a continuous network of cells throughout the collagen gel. In contrast, the FAK-KO MEFs formed round cellular clusters that varied in size throughout the whole depth of the gel (Figure 2B). FAK-KO MEFs also formed tube-like structures; however, 
upon further investigation, they consist of a string of individual cells and were not a single spread cell (data not shown). In order to examine cell morphology in more detail, samples were processed for electron microscopy. Semi-thin sections revealed thin, elongated FAK-WT cells that were interspersed in the gel where as FAK-KO MEFs were single, rounded cells that came together to form large cellular clusters (Figure 2C).

Since myosin II activation still occurred in FAK-KO MEFs, alterations in focal adhesion integrity could disrupt the mechanical linkage of the contractile machinery with the extracellular matrix, directly effecting the transmission of cellular tension and thus cell contraction. Therefore, focal adhesion formation was investigated in FAK-WT and FAK-KO MEFs in 3-D collagen gels by indirect immunofluorescence staining for vinculin. Thick, elongated plaques of vinculin could be seen at the ends of the actin stress fibers in FAK-WT MEFs indicating the formation of focal adhesions (Figure 3A). In FAK-KO MEFs, the abundance of vinculin staining was around the periphery co-localizing with actin filaments but there was no distinct focal adhesion plaque formation observed (Figure 3A). Thus, loss of FAK altered fibroblast cell morphology and focal adhesion formation in both 2-D and 3-D.

Focal adhesions are linked to the external cellular environment through heterodimeric, transmembrane receptors called integrins, which are comprised of an alpha $(\alpha)$ and beta $(\beta)$ chain. The combination of the two chains governs the specificity of the extracellular matrix protein it binds to. Since we did not observe classical focal adhesion formation in FAK-KO MEFs in 3-D, we wanted to investigate if the loss of FAK altered integrin protein levels. In 2-D, loss of FAK resulted in an increase in $\alpha_{1}$-integrin, a decrease in $\alpha_{5}$-integrin, and no real change in $\alpha_{\mathrm{V}}$-integrin protein levels, while in 3-D, there was a decrease in $\alpha_{1}$-integrin and $\alpha_{5}$-integrin, and

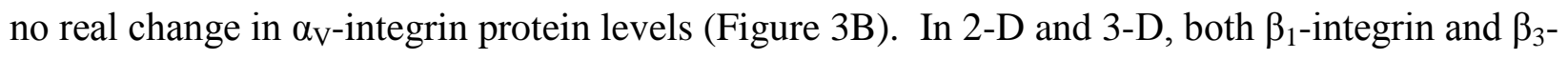


integrin remained unaltered with the loss of FAK however, in 3-D, there was almost no $\beta_{1^{-}}$ integrin and very low levels of $\beta_{3}$-integrin protein present in both cell lines (Figure $3 \mathrm{C}$ ) indicating a switch in the integrins that may be presented on the cell surface in 3-D.

\section{Loss of FAK results in the inability to compress the collagen gel}

Upon observation of cell morphology within the collagen gels, there appeared to be a significant difference in the thickness of the FAK-KO collagen gels compared to controls and therefore measurements of collagen gel thickness was performed using two-photon microscopy. Gels consisting of collagen alone formed a gel that was approximately $1.2 \mathrm{~mm}$ thick, however control and FAK-WT MEFs compressed the collagen down to a $419 \mu \mathrm{m}$ and $406 \mu \mathrm{m}$ thick gel, respectively. In comparison, FAK-KO MEFs were only able to form a $770 \mu \mathrm{m}$ thick gel, indicating that FAK-KO MEFs demonstrate an impaired ability to compress the collagen (Fig 4). In order to ensure that the difference in the thickness of the collagen gel was not due to FAK-KO cell clumping, FAK-KO MEFs embedded in collagen gels were stained with Hoescht 33258 dye and rhodamine phalloidin to visualize cell nuclei and actin, respectively, one to four days postseeding. Twenty-four hours post-seeding, FAK-KO MEFs were evenly dispersed throughout the depth of the collagen gel (Supplementary Figure 2). As the collagen gels were incubated and allowed to grow, clusters of FAK-KO MEFs forming throughout the collagen gel could be seen, resulting in the formation in large cellular clusters four days post-seeding (Supplementary Figure 2). Therefore, the FAK-KO MEFs formed large cellular clusters over a period of 4 days indicating cells were evenly distributed upon casting and the cell clusters appear to form as cells divide and migrate, aggregating cells into clusters. 


\section{FAK is necessary for collagen matrix organization in 3-D}

The lack of classical focal adhesion formation in FAK-KO MEFs in 3-D, differences in integrin protein expression, and the inability to compress the collagen gel suggests that the complex that links the internal and external cellular environments could be compromised. In order for the cell to contract, it has to exert force on the surrounding collagen fibrils and organize the collagen matrix. Defects in the focal adhesion complex would disrupt the transmission of force to the matrix and impair the ability for the cell to arrange the collagen fibers and result in cell contraction. Therefore, how the loss of FAK affects the ability of the cells to organize the collagen matrix was determined through analysis of the structure of the collagen matrix. Imaging of the collagen matrix was performed using a two-photon scattering method called second harmonic generation (SHG) microscopy, which is a nonlinear method to image light scattering. It is possible to utilize this technique to image the collagen matrix due to the collagen filaments exhibiting a high degree of molecular order and are noncentrosymmetric, meaning they have no molecular symmetry about a central point (Murphy and Davidson, 2013). Imaging of collagen gels alone without cells revealed a low SHG signal that was uniformly distributed throughout the image (Figure 5A). Collagen fibers in FAK-WT collagen gels generated areas of strong SHG signal throughout the image indicating the organization and uniform structure of the collagen fibers (Figure 5A). However, the collagen matrix within FAK-KO collagen gels produced a very low, uniform SHG signal, much like collagen alone (Figure 5A) indicating a lack of collagen organization and bundling. Quantitation of pixel intensity revealed collagen within FAK-WT gels exhibited a stronger second harmonic signal (82.46 \pm 4.41 pixels) compared to collagen alone and FAK-KO collagen gels $(58.12 \pm 2.765$ and $35.76 \pm 2.75$ pixels, respectively) (Figure 5B). 
To gain a more detailed picture of the collagen matrix, collagen gels were processed and imaged using scanning electron microscopy (SEM). A SEM micrograph of 3-D collagen alone showed a random, nonhomeogenous arrangement of collagen fibers. However, when FAK-WT MEFs were present within the collagen gel, they formed a highly organized, dense network of collagen matrix with bundling of the collagen fibers (Figure 6A). In comparison, FAK-KO MEFs appeared to be unable to organize the collagen matrix, resulting in a loose arrangement of collagen fibers that lacked a distinct organizational pattern, much like collagen alone (Figure 6A). Since immersion fixation and processing allows one to only image the surface of the collagen gels, a freeze-fracture procedure was performed on the samples to image the collagen matrix deeper within the gel. The collagen fibers within the FAK-WT collagen gels were densely packed together surrounding the cells and completely engulfing each cell where as the collagen fibers in the FAK-KO collagen gels were loosely arranged around the cellular clusters that formed (Figure 6B). Upon fracture of the sample, the FAK-KO MEFs disassociated from the collagen, leaving behind small craters within the collagen matrix due to the loose association with the collagen fibers (data not shown). Therefore, the loss of FAK impaired the ability for the cell to functionally interact with and organize the collagen.

\section{Rescue of FAK expression reverses FAK knockout phenotype}

In order to determine if FAK is critical for force generation and matrix organization, FAK-KO MEFs were infected with a lentivirus expressing either a wild-type form of FAK (WTFAK) or a constitutively active form of FAK (FAK180/3) to rescue the FAK knockout. Expression of WT-FAK or FAK180/3 restored the protein levels of FAK back to control levels (Figure 7A) and reversed the increase in Pyk2 protein levels seen in FAK-KO MEFs (data not 
shown). In order to determine if the FAK knockout phenotype had been restored, tension measurement of the re-expressing cells was performed. Interestingly, basal tension was not restored in WT-FAK and FAK180/3 and was similar to FAK-KO MEFs (Figure 7B). Following establishment of stable basal tension, MEFs were stimulated with LPA. FAK-WT MEFs produced a 110 dyne increase in tension. FAK-KO MEFs produced a 20 dyne increase in tension where as the WT-FAK and FAK180/3 MEFs produced a 160 dyne and 200 dyne increase in tension, respectively, indicating that the inability to produce force in the presence of an agonist exhibited by the FAK-KO MEFs was restored (Figure 7B). Cell morphology of rescued cells in 2-D culture was reversed from the knockout phenotype showing a spread cell morphology with a number of cellular protrusions compared to the round cell morphology exhibited by FAK-KO MEFs (Supplementary Figure 3). The rescued cells also had thin, elongated focal adhesion plaques, which were mainly around the periphery much like control and FAK-WT MEFs (Supplementary Figure 3). There was no apparent difference in morphology or focal adhesion distribution between WT-FAK and FAK180/3 MEFs in 2-D. Since 2-D cell morphology and focal adhesion distribution was reversed with re-expression of FAK in FAK-KO MEFs, we determined if the 3-D morphology was altered in the rescued cell lines. WT-FAK and FAK180/3 MEFs were grown in 3-D collagen gels and stained for vinculin. Both WT-FAK and FAK180/3 cell lines spread and formed an interconnected sheet of cells in 3-D compared to the large cellular clusters formed by FAK-KO MEFs. Vinculin immunofluorescence staining revealed distinct plaques at the ends of the actin filaments indicating the formation of focal adhesions within the rescued cells (Figure 7C) and restoration of both cell morphology and focal adhesion formation in the rescue cell lines. 
Since the ability to produce force was re-established in the rescued cell lines, we wanted to see if the ability to compress the collagen gel was restored as well. Measurement of overall gel thickness revealed WT-FAK and FAK180/3 compressed the collagen gel down to a $384 \mu \mathrm{m}$ and $325 \mu \mathrm{m}$ thick gel, respectively, which was greater than a 50\% decrease in FAK-KO gel depth and comparable to control and FAK-WT gel thickness (Figure 7D) indicating that the rescue of the knockout phenotype restored the ability for the MEFs to compress the collagen.

Since the rescued cell lines showed the formation of focal adhesions, we wanted to see if the impairment of functional interaction with collagen seen with the FAK-KO MEFs could be reversed. WT-FAK and FAK180/3 MEFs were grown in 3-D collagen gels and collagen organization was visualized using SHG microscopy. Regions of strong SHG signal were detected in 3-D gels comprised of both rescued cell lines (Supplementary Figure 4A). Quantitation of pixel intensity revealed WT-FAK and FAK180/3 collagen gels had an average pixel intensity similar to FAK-WT collagen gels $(83.52 \pm 3.12,93.4 \pm 5.04$ versus $82.46 \pm 4.41$ pixels, respectively) indicating the ability to organize the collagen had been restored (Supplementary Figure 4B). To confirm these findings, SEM was performed on the 3-D collagen gels. Both rescue cell lines exhibited a highly organized, dense collagen matrix which appeared to be similar to that in FAK-WT collagen gels (Figure 8) indicating a reversal of FAKKO MEFs inability to organize the collagen fibers and the importance of FAK in 3-D collagen matrix organization.

\section{FAK kinase activity is not necessary for tension generation}

FAK is an important nonreceptor tyrosine kinase that is involved in the signaling of many physiology processes, and therefore, FAK kinase activity could be important in the regulation of 
tension generation. In order to determine if the enzymatic activity of FAK was necessary for contractile force, a small molecule inhibitor of FAK kinase, PF-573,228 (Slack-Davis et al. 2007), was utilized. Time-course studies revealed the greatest inhibition of FAK kinase activity, as measured by phosphorylated tyrosine residue 397 of FAK, occured after 15 minutes of treatment (Figure 9A). Following establishment of stable basal tension, FAK-WT and FAK-KO MEFs were treated with either DMSO or $1 \mu \mathrm{M}$ PF-573,228 for 15 minutes (Figure 9A). Inhibition of FAK kinase activity caused a slight increase in FAK-WT tension and a minimal reduction in FAK-KO tension. The presence of DMSO had no effect on force. Inhibition of FAK kinase activity had no effect on the increase in LPA induced FAK-WT tension, resulting in a 100 dyne increase in tension which was comparable to untreated FAK-WT levels (Figure 9A). In contrast, LPA treatment only induced a 10 dyne increase in FAK-KO tension for both DMSO and PF-573,228 treated cells indicating that the inhibitor was not affecting any other molecule that may be involved in agonist induced tension generation (Figure 9A). Tension generated in response to LPA was abolished by application of $1 \mu \mathrm{M}$ CytoD.

To confirm that FAK kinase activity was not involved in tension generation, a kinase dead mutant form of FAK was expressed in FAK-KO MEFs through adenoviral infection (FAKdead). Comparable protein levels of FAK were seen in FAK-dead and FAK-WT MEFs (Figure 9B). Infected cells were embedded in collagen gels and hung from force transducers as previously described. FAK-dead MEFs had a similar basal force as FAK-WT MEFs. Stimulation with LPA induced a 90 dyne increase in both FAK-dead and FAK-WT MEFs tension (Figure 9B). Therefore, FAK kinase activity is not necessary for tension generation. Interestingly, there was a robust amount of phosphorylated FAK in cells seeded on collagencoated dishes, however, FAK was not phosphorylated in 3-D indicating that FAK was not 
activated under basal conditions and does not appear to be critical for cell tension generation in 3-D (Figure 9C).

Phosphorylation of FAK recruits and activates the Src-family of kinases to the focal adhesions resulting in activation of FAK and downstream signaling cascades (Schaller et al., 1994; Xing et al., 1994). However, the Src-family of kinases can be activated independently of FAK binding and regulate cell function. Therefore, we wanted to determine if the activity of the Src-family kinases was critical for tension generation. Following establishment of stable basal tension, FAK-WT MEFs were treated with either DMSO or $1 \mu \mathrm{M}$ of the Src-family kinase inhibitors PP2 or SU6656 for 15 minutes. Inhibition of the activity of the Src-family kinases did not alter FAK-WT basal and agonist-induced tension generation (Supplementary Figure 5) indicating the Src-family kinases were not critical in the regulation of tension generation.

\section{FAK targeting to focal adhesions is necessary for tension generation}

Since FAK was critical for force generation but FAK kinase activity was not important, we wanted to determine if FAK targeting to the focal adhesion was needed for basal and agonist induced force. In order to disrupt FAK localization to focal adhesions, cells were grown in collagen gels while in the presence of the small molecule chloropyramine hydrochloride (C4), which blocked FAK targeting to focal adhesions (Kurenova et al., 2009). MEFs treated with C4 had a low basal force compared to FAK-WT MEFs. Treatment with LPA induced a rapid rise in FAK-WT tension generation, however, there was no increase in tension in C4 treated MEFs (Figure 9D) indicating loss of FAK localization to the focal adhesions altered tension generation.

To ensure FAK localization to focal adhesions was critical for tension generation, a mutant form (FRNK-C1034S) of a naturally occurring variant of FAK called FRNK (FAK- 
related non-kinase), was expressed in FAK-KO MEFs. FRNK is an endogenously expressed variant of FAK which is composed of the noncatalytic C-terminal region of FAK and acts as a dominant-negative inhibitor of FAK (Schaller et al., 1993; Richardson and Parsons, 1996; Nolan et al., 1999). FRNK localizes to the focal adhesions however does not have any kinase activity. FRNK-C1034S (Bryant et al., 2006) has residue 1034 in the FAT domain mutated, disrupting paxillin binding and localization within focal adhesions (Sieg et al., 1999, Tomar et al., 2009). Therefore, FRNK-C1034S MEFs should not replace FAK within the focal adhesions and will result in minimal force generation. FRNK MEFs produced a similar basal force and agonist induced force to FAK-WT MEFs, however, expression of FRNK-C1034S resulted in low basal force and minimal agonist induced force indicating that FAK localization to focal adhesions was critical for both basal and agonist induced tension generation (Figure 9E). Thus, the FAT domain and not kinase activity of FAK is critical for force production.

\section{Discussion}

In the present study, FAK knockout fibroblasts were utilized to define the role of FAK in fibroblast basal and agonist induced tension generation in 3-D. We report that loss of FAK resulted in: 1) reduced basal force, 2) minimal agonist induced tension generation, 3) no alterations in myosin II phosphorylation, 4) a lack of cell spreading in 2-D and 3-D, 5) an increase in focal adhesion formation in 2-D but a lack of classical focal adhesion formation in 3$\mathrm{D}, 6)$ the inability of the cell to organize the collagen fibers, and 7) the localization of FAK to the focal adhesions via its FAT domain is critical for the generation and transmission of force. Our findings indicate FAK acts in a kinase independent mechanism to facilitate transduction of 
cellular forces from inside to outside of the cell, resulting in organization of the collagen matrix and cell contraction.

Upon integrin engagement with ECM proteins, FAK is localized to focal adhesions via its FAT domain. Following autophosphorylation and activation of FAK, other adaptor and signaling proteins, such as paxillin, Src, and vinculin, are recruited to the focal adhesion to aid in the formation of the complex and regulate downstream signaling pathways. The focal adhesion is then linked up to the contractile machinery of actin and myosin through actin binding proteins, such as vinculin and talin, creating a nexus for the internal cytoskeleton with the external collagen fibers. The mechano-energy generated by the interaction of actin and myosin is transmitted through the focal adhesions to the collagen fibers through the focal adhesion complex. FAK is necessary for the proper arrangement of the adhesion proteins and focal adhesion linkage to the actin cytoskeleton to allow force to be transmitted through the adhesion to the collagen matrix. The transmission of force to the external ECM results in movement of the fibrils into an organized network of collagen fibers. Bundling and organizing the collagen increases the stiffness of the matrix and provides a rigid substrate for the cell to be able to hold onto and anchor itself. FAK is critical for the strengthening of this adhesion. The result is a strengthened adhesion that anchors the cell to the collagen matrix and provides a resisting force to the pulling force of the tensile actin stress fibers, resulting in cell contraction (Figure 10A).

When FAK is knocked out of the cell in 3-D (Figure 10B), recruitment of focal adhesion proteins does occur; however there appears to be an accumulation of the proteins without classical focal adhesion formation (Figure 3A). Also, in the absence of FAK, the recruitment of key focal adhesion proteins may be altered. Without the proper proteins present, the focal adhesions may not link to the actin cytoskeleton or are weakly connected. There is still an 
increase in myosin activation and interaction with the actin cytoskeleton (Figure 1C) however this association may be concentrated around the periphery due to the abundance of cortical actin (Figure 3A). An increase in internal tension occurs, however due to the actin cytoskeleton not being linked up properly, force cannot be transmitted through the adhesion to the collagen matrix resulting in the inability to organize the collagen fibers. A non-organized matrix is a more pliable substrate to bind to and the weakened adhesions cannot anchor the cell to the matrix properly. Thus, any cellular tension generated in the cell does not have a strong anchor to pull on in order to induce cell contraction (Figure 10B). Since FRNK is the C-terminal region of FAK, expression of FRNK (Figure 10C) in FAK-KO MEFs results in localization of FRNK to the focal adhesions. This causes a phenotype similar to control cells with the collagen matrix being organized into a dense network of collagen fibers and the induction of cell contraction (Figure 10A). FRNK has no catalytic activity suggesting that FAK kinase activity is not critical for tension generation. Expression of a mutant form of FRNK that does not localize to focal adhesions in FAK-KO MEFs (FRNK-C1034S) has a similar phenotype to the FAK-KO MEFs with lack of collagen matrix organization and minimal force generation (Figure 10D). Therefore, FAK localization in the focal adhesion is critical for force transmission through the focal adhesion complex, resulting in organization of the collagen fibers and increases in the strength of the focal adhesion, allowing cell contraction to occur.

Studies determining the role of FAK as a mechanotransducer and its involvement in the regulation of key contractile signaling pathways has been well documented in 2-D. However, investigations of how FAK is involved in cellular force generation and transmission are limited. FAK null fibroblasts exhibit a rounded cell morphology with enlarged focal adhesions and decreased cell motility (Ilic et al., 1995). Loss of FAK results in increased Rho, ROCK, and 
MLCK activity as well as increased myosin phosphorylation (Ren et al., 2000; Chen et al., 2002; Pirone et al., 2006; Schober et al., 2007). The current knowledge suggests that loss of FAK should lead to an increase in overall cellular tension. However, our findings show that loss of FAK resulted in a low basal tension and minimal force generation in the presence of an agonist without any alterations in myosin phosphorylation (Figure 1A-C). Previous studies determined the contractile state of the cell by measuring phosphorylation status of proteins or utilizing kinase inhibitors and determining how that affected cell phenotype without direct measurement of changes in kinase activity or overall cell contractility. Also, most studies focused on single cell preparations or cells seeded onto substrate-coated dishes. Cell behavior does differ in 3-D and therefore it is difficult to make direct comparisons between this study and others. Similarly to our findings, Tang and Gunst (2001) directly measured contractility of smooth muscle cells and found that knockdown of FAK resulted in a decrease in intracellular calcium, myosin activity, and cell contractility. Conversely, a recent study in our lab showed that knockdown of FAK in endothelial cells resulted in an increase in force generation in 3-D (Arnold et al., 2013). Therefore, the role FAK plays in tension generation may differ depending on cell-type and conditions present.

To our knowledge, this is the first documentation of FAK being critical for collagen matrix organization in 3-D. FAK has been shown to play a role in the organization of other ECM proteins, such as fibronectin. Integrin binding initiates responses within the cell that makes it possible to organize a fibrillar fibronectin network (Wu et al., 1995). However, integrin engagement is not sufficient for fibronectin matrix assembly but rather requires cells to exert tension on the ECM through linkage of actin stress fibers with the focal adhesions in order to organize the fibronectin matrix (Zhong et al., 1998; Schoenwaelder and Burridge, 1999; Sechler 
et al., 2001; Lemmon et al., 2009). Due to the localization of FAK in the focal adhesions and its proximity to the plasma membrane, it has become a molecule of interest in fibronectin matrix assembly. Endothelial cells, fibroblasts, and mouse embryos lacking FAK formed a less fibrillar and more punctate fibronectin matrix suggesting that loss of FAK impaired fibronectin organization. FAK KO resulted in a poorly organized fibronectin network with short fibril formation and the inability to coalign fibrils with actin filaments at adhesion sites. This altered matrix was not due to changes in synthesis of fibronectin but the cell's ability to organize the matrix (Ilic et al, 2004). Cells can also actively rearrange their fibrillar collagen in a forcedependent manner (Li et al., 2003; Canty and Kadler, 2005; Canty et al., 2006). Therefore, FAK is necessary for force transmission through the focal adhesion in order to organize the collagen fibrils. Also, loss of FAK significantly weakened integrin-ECM adhesion strength impairing the ECM-cytoskeleton connection (Klemm et al., 2009). An intact actin cytoskeletal connection with the ECM via the focal adhesions has been shown to be crucial for cell stiffness and traction force generation (Fabry et al., 2011). We observed a lack of focal adhesion formation and stress fiber formation in FAK-KO MEFs in 3-D (Figure 3A). Therefore, post-adhesive cytoskeletal organization and associated adhesion site formation is a FAK-mediated process that supports normal collagen matrix organization.

FAK kinase activity has been documented extensively as being necessary for a variety of cellular processes, such as cell migration, growth, and survival (Schaller et al., 1992; Ilic et al., 1995; Ilic et al., 1998; Sieg et al., 1999; Sieg et al., 2000). In studies investigating contractile signaling pathways in 2-D, FAK phosphorylates critical enzymes that regulate myosin II activity and cytoskeletal dynamics (Chikumi et al. 2002; Webb et al., 2004; Holinstat et al., 2006). However, this study shows FAK kinase activity is dispensable for tension generation through 
small molecule inhibition and mutant constructs. We therefore cannot underscore the importance of FAK localization to the focal adhesion in regulating cell behavior, especially in 3 D culture. FAK has been implicated in playing a role in many disease processes including cardiovascular complications, fibrosis, and tumor formation and metastasis. (Gabarra-Niecko et al., 2003; van Nimwegen et al., 2005; Braren et al., 2006; Peng et al., 2008; Wong et al., 2011; Lagares et al., 2012). Treatments for these diseases are focused on development of inhibitors of FAK kinase activity. However, the efficacy of these inhibitors in human trials is still under investigation (Dunn et al., 2010). Mechanical properties of the cell also play a key role in the progression of these diseases. Development of localized inhibitors or cell specific inhibition of FAK translocation to the focal adhesion may mitigate the progression of diseases and be an effective route in the development of treatments targeting FAK.

\section{Acknowledgements}

We would like to thank the following: Dr. Kevin Pumiglia for supplying the mutant FAK adenoviruses; Dr. Michael Schaller for scientific input and supplying the WT-FAK and FAK180/3 lentiviral constructs; and Graphic Deisgns by J.R. Arnold, LLC for drawing the illustration for Figure 10. Imaging experiments and image analysis were performed in the West Virginia University Microscope Imaging Facility, which has been supported by the Mary Babb Randolph Cancer Center and NIH grants P20 RR016440, P30 RR032138/GM103488 and P20 RR016477. This research was supported by NIH grants: HL-45788, HL-90937, P20-RR16440 and Cardiovascular and Pulmonary Disease T32 HL-090610. 


\section{References}

Amano, M., M. Ito, K. Kimura, Y. Fukata, K. Chihara, T. Nakano, Y. Matsuura, and K.

Kaibuchi. 1996. Phosphorylation and activation of myosin by Rho-associated kinase (Rhokinase). J. Biol. Chem. 271:20246-20249.

Arnold, K.M., Z.M. Goeckeler, and R.B. Wysolmerski. 2013. Loss of focal adhesion kinase enhances endothelial barrier function and increases focal adhesions. Microcirculation. doi: 10.1111/micc. 12063 .

Balaban, N.Q., U.S. Schwarz, D. Riveline, P. Goichberg, G. Tzur, I. Sabanay, D. Mahalu, S. Safran, A. Bershadsky, L. Addadi, and B. Geiger. 2001. Force and focal adhesion assembly: a close relationship studied using elastic micropatterned substrates. Nat. Cell Biol. 3:466-472.

Braren, R., H. Hu, Y.H. Kim, H.E. Beggs, L.F. Reichardt, and R. Wang. 2006. Endothelial FAK is essential for vascular network stability, cell survival, and lamellipodial formation. $J$. Cell Biol. 172:151-162.

Bryant, P., Q. Zheng, and K. Pumiglia. 2006. Focal adhesion kinase controls cellular levels of p27/Kip1 and p21/Cip1 through Skp2-dependent and -independent mechanisms. Mol. Cell Biol. 26:4201-4213.

Burridge, K., K. Fath, T. Kelly, G. Nuckolls, and C. Turner. 1988. Focal adhesions: transmembrane junctions between the extracellular matrix and the cytoskeleton. Annu. Rev. Cell Biol. 4:487-525.

Canty, E.G., and K.E. Kadler. 2005. Procollagen trafficking, processing and fibrillogenesis. $J$. Cell Sci. 118:1341-1353.

Canty, E.G., T. Starborg, Y. Lu, S.M. Humphries, D.F. Holmed, R.S. Meadows, A. Huffman, E.T. O'Toole, and K.E. Kadler. 2006. Actin filaments are required for fibripositor-mediated collagen fibril alignment in tendon. J. Biol. Chem. 281:38592-38598.

Chen, B.H., J.T. Tzen, A.R. Bresnick, and H.C. Chen. 2002. Roles of Rho-associated kinase and myosin light chain kinase in morphological and migratory defects of focal adhesion kinasenull cells. J. Biol. Chem. 277:33857-33863.

Chew, T.L., R.A. Masaracchia, Z.M. Goeckeler, and R.B. Wysolmerski. 1998. Phosphorylation of non-muscle myosin II regulatory light chain by p21-activated kinase (gamma-PAK). J. Muscle Res. Cell Motil. 19:839-854.

Chikumi, H., S. Fukuhara, and J.S. Gutkind. 2002. Regulation of G protein-linked guanine nucleotide exchange factors for Rho, PDZ-RhoGEF, and LARG by tyrosine phosphorylation: evidence of a role for focal adhesion kinase. J. Biol. Chem. 277:12463-12473.

Connor, D.A. 2000. Mouse embryo fibroblast (MEF) feeder cell preparation. Curr. Protoc. Mol. Biol. Unit 23.2:23.2.1-23.2.7. 
Cukierman, E., R. Pankov, D.R. Stevens, and K.M. Yamada. 2001. Taking cell-matrix adhesions to the third dimension. Science. 294:1708-1712.

Dunn, K.B., M. Heffler, and V.M. Golubovskaya. 2010. Evolving therapies and FAK inhibitors for the treatment of cancer. Anticancer Agents Med. Chem. 10:722-734.

Emmert, D.A., J.A. Fee, Z.M. Goeckeler, J.M. Grojean, T. Wakatsuki, E.L. Elson, B.P. Herring, P.J. Gallagher, and R.B. Wysolmerski. 2004. Rho-kinase mediated Ca2+-independent contraction in rat embryo fibroblasts. Am. J. Physiol. Cell Physiol. 286:C8-C21.

Fabry, B., A.H. Klemm, S. Kienle, T.E. Schaffer, and W.H. Goldmann. 2011. Focal adhesion kinase stabilizes the cytoskeleton. Biophys. J. 101:2131-2138.

Gabarra-Niecko, V., M.D. Schaller, and J.M. Dunty. 2003. FAK regulates biological processes important for the pathogenesis of cancer. Cancer Metastasis Rev. 22:359-374.

Giloh, H., and J.W. Sedat. 1982. Fluorescence microscopy: reduced photobleaching of rhodamine and fluorescein protein conjugates by n-propyl gallate. Science. 217:1252-1255.

Goeckeler, Z.M., P.C. Bridgman, and R.B. Wysolmerski. 2008. Nonmuscle myosin II is responsible for maintaining endothelial cell basal tone and stress fiber integrity. Am. J. Physiol. Cell Physiol. 295:C994-C1006.

Goeckeler, Z.M., and R.B. Wysolmerski. 1995. Myosin light chain kinase-regulated endothelial cell contraction: the relationship between isometric tension, actin polymerization, and myosin phosphorylation. J. Cell Biol. 130:613-627.

Guan, J-L., J.E. Trevithick, and R.O. Hynes. 1991. Fibronectin/integrin interaction induces tyrosine phosphorylation of a 120-kDa protein. Cell Regul. 2:951-964.

Hanks, S.K., M.B. Calalb, M.C. Harper, and S.K. Patel. 1992. Focal adhesion protein-tyrosine kinase phosphorylated in response to cell attachment to fibronectin. Proc. Natl. Acad. Sci. USA. 89:8487-8491.

Holinstat, M., N. Knezevic, M. Broman, A.M. Samarel, A.B. Malik, and D. Mehta. 2006. Suppression of RhoA activity by focal adhesion kinase-induced activation of p190RhoGAP: role in regulation of endothelial permeability. J. Biol. Chem. 281:2296-2305.

Hynes, R.O. 2002. Integrins: bidirectional, allosteric signaling machines. Cell. 110:673-687. Ilic, D., Almeida, E.A., Schlaepfer, D.D., Dazin, P., Aizawa, S., and C.H. Damsky. 1998. Extracellular matrix survival signals transduced by focal adhesion kinase suppress p53-mediated apoptosis. J. Cell Biol. 143:547-560. 
Ilic, D., Y. Furuta, S. Kanazawa, N. Takeda, K. Sobue, N. Nakatsuji, S. Nomura, J. Fujimoto, M. Okada, and T. Yamamoto. 1995. Reduced cell motility and enhanced focal adhesion contact formation in cells from FAK-deficient mice. Nature. 377:539-544.

Ilic, D., B. Kovacic, K. Johkura, D.D. Schlaepfer, N. Tomasevic, Q. Han, J.B. Kim, K. Howerston, C. Baumbusch, N. Ogiwara, D.N. Streblow, J.A. Nelson, P. Dazin, Y. Shino, K. Sasaki, and C.H. Damsky. 2004. Fak promotes organization of fibronectin matrix and fibrillar adhesions. J. Cell Sci. 117:177-187.

Ilic, D., B. Kovacic, S. McDonagh, F. Jin, C. Baumbusch, D.G. Gardner, and C.H. Damsky. 2003. Focal adhesion kinase is required for blood vessel morphogenesis. Circ. Res. 92:300307.

Jockusch, B.M., P. Bubeck, K. Giehl, M. Kroemker, J. Moschner, M. Rothkegel, M. Rudiger, K. Schluter, G. Stanke, and J. Winkler. 1995. The molecular architecture of focal adhesions. Аnпu. Rev. Cell Dev. Biol. 11:379-416.

Kanner, S.B., A.B. Reynolds, R.R. Vines, and J.T. Parsons. 1990. Monoclonal antibodies to individual tyrosine-phosphorylated protein substrates of oncogene-encoded tyrosine kinases. Proc. Natl. Acad. Sci. USA. 87:3328-3332.

Kawano, Y., Y. Fukata, N. Oshiro, M. Amaon, T. Nakamura, M. Ito, F. Matsumura, M. Inagaki, and K. Kaibuchi. Phosphorylation of myosin-binding subunit (MBS) of myosin phosphatase by Rho-kinase in vivo. J. Cell Biol. 147:1023-1038.

Kimura, K., M. Ito, M. Amano, K. Chihara, Y. Fukata, M. Nakafuku, B. Yamamori, J. Feng, T. Nakano, K. Okawa, A. Iwamatsu, and K. Kaibuchi. 1996. Regulation of myosin phosphatase by Rho and Rho-associated kinase (Rho-kinase). Science. 273:245-248.

Klemm, A.H., G. Diez, J-L. Alonso, and W.H. Goldmann. 2009. Comparing the mechanical influence of vincluin, focal adhesion kinase and p53 in mouse embryonic fibroblasts. Biochem. Biophys. Res. Commun. 379:799-801.

Kolodney, M.S., and R.B. Wysolmerski. 1992. Isometric contraction by fibroblasts and endothelial cells in tissue culture: a quantitative study. J. Cell Biol. 117:73-82.

Kornberg, L.J., H.S. Earp, C.E. Turner, C. Prockop, and R.L. Juliano. 1991. Signal transduction by integrins: increased protein tyrosine phosphorylation caused by clustering of beta 1 integrins. Proc. Natl. Acad. Sci. USA. 88:8392-8396.

Kureishi, Y., S. Kobayashi, M. Amano, K. Kimuar, H. Kanaide, T. Nakano, K. Kaibuchi, and M. Ito. 1997. Rho-associated kinase directly induces smooth muscle contraction through myosin light chain phosphorylation. J. Biol. Chem. 272:12257-12260.

Kurenova, E.V., D.L. Hunt, D. He, A.T. Magis, D.A. Ostrov, and W.G. Cance. 2009. Small molecule chloropyramine hydrochloride (C4) targets the binding site of focal adhesion kinase 
and vascular endothelial growth factor receptor 3 and suppresses breast cancer growth in vivo. $J$. Med. Chem. 52:4716-4724.

Lagares, D., O. Busnadiego, R.A. Garcia-Fernandez, M. Kapoor, S. Liu, D.E. Carter, D. Abraham, X. Shi-Wen, P. Carreira, B.A. Fontaine, B.S. Shea, A.M. Tager, A. Leask, S. Lamas, and F. Rodriguez-Pascual. 2012. Inhibition of focal adhesion kinase prevents experimental lung fibrosis and myofibroblast formation. Arthritis Rheum. 64:1653-1654.

Lemmon, C.A., C.S. Chen, and L.H. Romer. 2009. Cell traction forces direct fibronectin matrix assembly. Biophys. J. 96:729-738.

Li, S., M. Kim, Y.L. Hu, S. Jalali, D.D. Schlaepfer, T. Hunter, S. Chien, and J.Y. Shyy. 1997. Fluid shear stress activation of focal adhesion kinase. Linking to mitogen-activated protein kinases. J. Biol. Chem. 272:30455-30462.

Li, S., C. van den Diepstraten, S.J. D’Souza, B.M.C. Chan, and J.G. Pickering. 2003. Vascular smooth muscle cells orchestrate the assembly of type I collagen via alpha2beta1 integrins, RhoA, and fibronectin polymerization. Am. J. Pathol. 163:1045-1056.

Lietha, D., X. Cai, D.F. Ceccarelli, Y. Li, M.D. Schaller, and M.J. Eck. 2007. Structural basis for the autoinhibition of focal adhesion kinase. Cell. 129:1177-1187.

Lim, Y., S.T. Lim, A. Tomar, M. Gardel, J.A. Bernard-Trifilo, X.L. Chen, S.A. Uryu, R. CaneteSoler, J. Zhai, H. Lin, W.W. Schlaepfer, P. Nalbant, G. Bokoch, D. Ilic, C. Waterman-Storer, and D.D. Schlaepfer. 2008. PyK2 and FAK connections to p190Rho guanine nucleotide exchange factor regulate RhoA activity, focal adhesion formation, and cell motility. J. Cell Biol. 180:187-203.

Liu, S., D.A. Calderwood, and M.H. Ginsberg. 2000. Integrin cytoplasmic domain-binding proteins. J. Cell Sci. 113:3563-3571.

Nolan, K., J. Lacoste, and J.T. Parsons. 1999. Regulated expression of focal adhesion kinaserelated nonkinase, the autonomously expressed $\mathrm{C}$-terminal domain of focal adhesion kinase. Mol. Cell Biol. 19:6120-6129.

Owen, J.D., P.J. Ruest, D.W. Fry, and S.K. Hanks. 1999. Induced focal adhesion kinase (FAK) expression in FAK-null cells enhances cell spreading and migration requiring both auto- and activation loop phosphorylation sites and inhibits adhesion-dependent tyrosine phosphorylation of Pyk2. Mol. Cell Biol. 19:4806-4818.

Parsons, J.T. 1993. Focal adhesion kinase: the first ten years. J. Cell Sci. 116:1409-1416.

Peng, X., X. Wu, J.E. Druso, H. Wei, A.Y. Park, M.S. Kraus, A. Alcaraz, J. Chen, S. Chien, R.A. Cerione, and J.L. Guan. 2008. Cardiac developmental defects and eccentric right ventricular hypertrophy in cardiomyocyte focal adhesion kinase (FAK) conditional knockout mice. Proc. Natl. Acad. Sci. USA. 105:6638-6643. 
Pirone, D.M., W.F. Liu, S.A. Ruiz, L. Gao, S. Raghaven, C.A. Lemmon, L.H. Romer, and C.S. Chen. 2006. An inhibitory role for FAK in regulating proliferation: a link between limited adhesion and RhoA-ROCK signaling. J. Cell Biol. 174:277-288.

Ren, X.D., W.B. Kiosses, D.J. Sieg, C.A. Otey, D.D. Schlaepfer, and M.A. Schwartz. 2000. Focal adhesion kinase suppresses Rho activity to promote focal adhesion turnover. J. Cell Sci. 113:3673-3678.

Renshaw, M.W., L.S. Price, and M.A. Schwartz. 1999. Focal adhesion kinase mediates the integrin signaling requirement for growth factor activation of MAP kinase. J. Cell Biol. 147:611-618.

Richardson, A., and J.T. Parsons. 1995. Signal transduction through integrins: a central role for focal adhesion kinase? Bioessays. 17:229-236.

Richardson, A., and J.T. Parsons. 1996. A mechanism for regulation of the adhesion-associated protein tyrosine kinase pp125FAK. Nature. 380:538-540.

Riveline, D., E. Zamir, N.Q. Balaban, U.S. Schwarz, T. Ishizaki, S. Narumiya, Z. Kam, B. Geiger, and A.D. Bershadsky. 2001. Focal contacts as mechanosensors: externally applied local mechanical force induces growth of focal contacts by an mDia1-dependent and ROCKindependent mechanism. J. Cell Biol. 153:1175-1186.

Sawada, Y., and M.P. Sheetz. 2002. Force transduction by Triton cytoskeletons. J. Cell Biol. 156:609-615.

Schaller, M.D., C.A. Borgman, B.S. Cobb, R.R. Vines, A.B. Reynolds, and J.T. Parsons. 1992. pp125FAK, a structurally distinctive protein-tyrosine kinase associated with focal adhesions. Proc. Natl. Acad. Sci. USA. 89:5192-5196.

Schaller, M.D., C.A. Borgman, and J.T. Parsons. 1993. Autonomous expression of a noncatalytic domain of the focal adhesion-associated protein tyrosine kinase pp125FAK. Mol. Cell Biol. 13:785-791.

Schaller, M.D., J.D. Hildebrand, J.D. Shannon, J.W. Fox, R.R. Vines, and J.T. Parsons. 1994. Complex formation with focal adhesion kinase: Autophosphorylation of the focal adhesion kinase pp125FAK directs SH2-dependent binding of pp60src. Mol. Biol. Cell. 14:1680-1688.

Schaller, M.D., and J.T. Parsons. 1994. Focal adhesion kinase and associated proteins. Curr. Opin. Cell Biol. 6:705-710.

Schober, M., S. Raghaven, M. Nikolova, L. Polak, H.A. Pasolli, H.E. Beggs, L.F. Reichardt, and E. Fuchs. 2007. Focal adhesion kinase modulates tension signaling to control actin and focal adhesion dynamics. J. Cell Biol. 176:667-680. 
Schoenwaelder, S.M., and K. Burridge. 1999. Bidirectional signaling between the cytoskeleton and integrins. Curr. Opin. Cell Biol. 11:274-286.

Scholey, J.M., K.A. Taylor, and J. Kendrick-Jones. 1980. Regulation of non-muscle myosin assembly by calmodulin-dependent light chain kinase. Nature. 287:233-235.

Sechler, J.L., H. Rao, A.M. Cumiskey, I. Vega-Colon, M.S. Smith, T. Murata, and J.E. Schwarzbauer. 2001. A novel fibronectin binding site required for fibronectin fibril growth during matrix assembly. J. Cell Biol. 154:1081-1088.

Sieg, D.J., C.R. Hauck, D. Ilic, C.K. Klingbeil, E. Schaefer, C.H. Damsky, and D.D. Schlaepfer. 2000. FAK integrates growth-factor and integrin signals to promote cell migration. Nat. Cell Biol. 2:249-256.

Sieg, D.J., C.R. Hauck, and D.D. Schlaepfer. 1999. Required role of focal adhesion kinase (FAK) for integrin-stimulated cell migration. J. Cell Sci. 112:2677-2691.

Slack-Davis, J.K., K.H. Martin, R.W. Tilghman, M. Iwanicki, E.J. Ung, C. Autry, M.J. Luzzio, B. Cooper, J.C. Kath, W.G. Roberts, and J.T. Parsons. 2007. Cellular characterization of a novel focal adhesion kinase inhibitor. J. Biol. Chem. 282:14845-14852.

Tan, J.L., S. Ravid, and J.A. Spudich. 1992. Control of nonmuscle myosins by phosphorylation. Annu. Rev. Biochem. 61:721-759.

Tang, D.D., and S.J. Gunst. 2001. Depletion of focal adhesion kinase by antisense depresses contractile activation of smooth muscle. Am. J. Physiol. Cell Physiol. 280:C874-C883.

Tomar, A., S.T. Lim, Y. Lim, and D.D. Schlaepfer. 2009. A FAK-p120RasGAP-p190RhoGAP complex regulates polarity in migrating cells. J. Cell Sci. 122:1852-1862.

Totsukawa, G., Y. Yamakita, S. Yamashiro, D.J. Hartshorne, Y. Sasaki, and F. Matsumura. 2000. Distinct roles of ROCK (Rho-kinase) and MLCK in spatial regulation of MLC phosphorylation for assembly of stress fibers and focal adhesions in 3 T3 fibroblasts. J. Cell Biol. 150:797-806.

Tsukada, T., Y. Tomooka, S. Takai, Y. Ueda, S. Nishikawa, T. Yagi, T. Tokunaga, N. Takeda, Y. Suda, and S. Abe. 1993. Enhanced proliferative potential in culture of cells from p53deficient mice. Oncogene. 8:3313-3322.

van Nimwegen, M.J., S. Verkoeijen, L. van Buren, D. Burg, and B. van de Water. 2005. Requirement for focal adhesion kinase in the early phase of mammary adenocarcinoma lung metastasis formation. Cancer Res. 65:4698-4706.

Webb, D.J., K. Donais, L.A. Whitmore, S.M. Thomas, C.E. Turner, J.T. Parsons, and A.F. Horwitz. 2004. FAK-Src signalling through paxillin, ERK and MLCK regulates adhesion disassembly. Nat. Cell Biol. 6:154-161. 
Webb, D.J., J.T. Parsons, and A.F. Horwitz. 2002. Adhesions assembly, disassembly and turnover in migrating cells - over and over and over again. Nat. Cell Biol. 4:E97-E100.

Weis, S.M., S.T. Lim, K.M. Lutu-Fuga, L.A. Barnes, X.L. Chen, J.R. Gothert, T.L. Shen, J.L. Guan, D.D. Schlaepfer, and D.A. Cheresh. 2008. Compensatory role for Pyk2 during angiogenesis in adult mice lacking endothelial cell FAK. J. Cell Biol. 181:43-50.

Wong, V.W., K.C. Rustad, S. Akaishi, M. Sorkin, J.P. Glotzbach, M. Januszyk, E.R. Nelson, K. Levi, J. Paterno, I.N. Vial, A.A. Kuang, M.T. Longaker, and G.C. Gurtner. 2011. Focal adhesion kinase links mechanical force to skin fibrosis via inflammatory signaling. Nat. Med. 18:148-152.

Wu, C., V.M. Keivens, T.E. O’Toole, J.A. McDonald, and M.H. Ginsberg. 1995. Integrin activation and cytoskeletal interaction are essential for the assembly of a fibronectin matrix. Cell. 83:715-724.

Xing, Z., H.C. Chen, J.K. Nowlen, S.J. Taylor, D. Shalloway, and J.L. Guan. 1994. Direct interaction of v-Src with the focal adhesion kinase mediated by the Src SH2 domain. Mol. Cell Biol. 5:413-421.

Yang, S., S. Adihikari, M. Dobbala, S. Adusumilli, J.D. Rowley, F. Ganikhanov, L. Zhang, G. Marrs, R. Wysolmerksi, and G. Spirou. 2013. Multi-color ultrafast laser platform for nonlinear optical imaging based on independently tunable optical parametric oscillators. Appl. Phys. B: Lasers and Optics. doi: 10.1007/s00340-013-5381-x.

Yang, S., R.B. Wysolmerksi, and F. Ganikhanov. 2011. Three-dimensional nonlinear microspectroscopy and imaging of soft condensed matter. Opt. Lett. 36:3849-3851.

Zaidel-Bar, R., and B. Geiger. 2010. The switchable integrin adhesome. J. Cell Sci. 123:13851388.

Zhao, J.H., H. Reiske, and J.L. Guan. 1998. Regulation of the cell cycle by focal adhesion kinase. J. Cell Biol. 143:1997-2008.

Zhong, C., M. Chrzanowska-Wodnicka, J. Brown, A. Shaub, A.M. Belkin, and K. Burridge. 1998. Rho-mediated contractility exposes a cryptic site in fibronectin and induces fibronectin matrix assembly. J. Cell Biol. 141:539:551. 

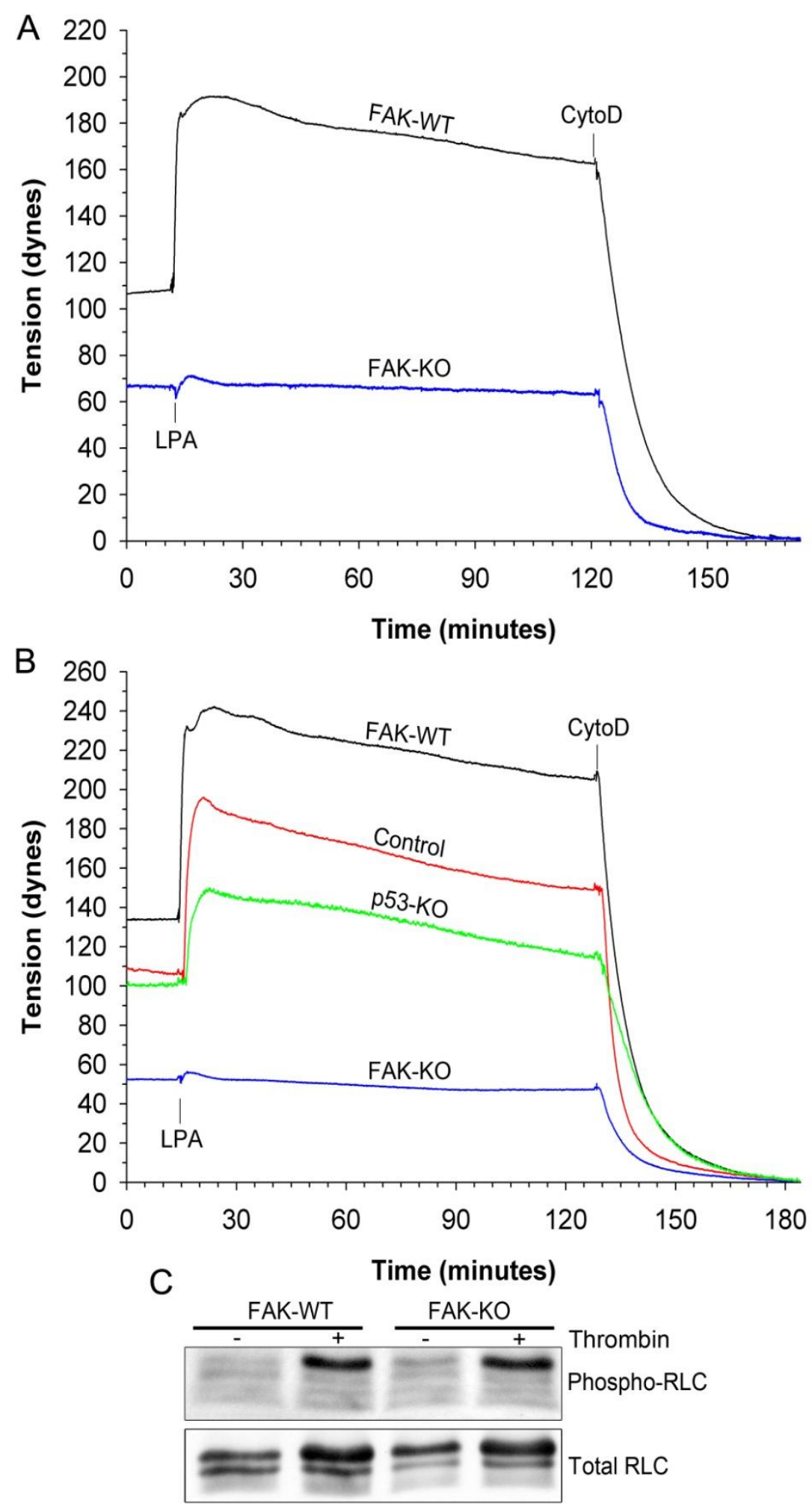

Figure 1 
Figure 1: Effect of FAK knockout on basal and agonist induced tension generation and myosin phosphorylation.

A) Representative force tracing of tension produced by FAK-WT (black) and FAK-KO (blue) MEFs treated with lysophosphatidic acid (LPA). Collagen gels were mounted on force transducers and allowed to establish a stable basal force before being stimulated with $1 \mu \mathrm{M} \mathrm{LPA}$. Loss of FAK resulted in reduced basal force and a minimal increase in agonist induced tension. (B) Representative force tracing of tension produced by control (red), p53-KO (green), FAK-WT (black), and FAK-KO (blue) MEFs treated with $1 \mu \mathrm{M}$ LPA. Both control and p53-KO MEFs produced tension in the presence of LPA similar to FAK-WT MEFs. (C) FAK-WT and FAKKO 3-D extracts were analyzed for phosphorylated myosin II RLC and total RLC under basal and agonist stimulated conditions. Reduction in cellular levels of FAK did not alter basal or agonist stimulated myosin RLC phosphorylation. 
A
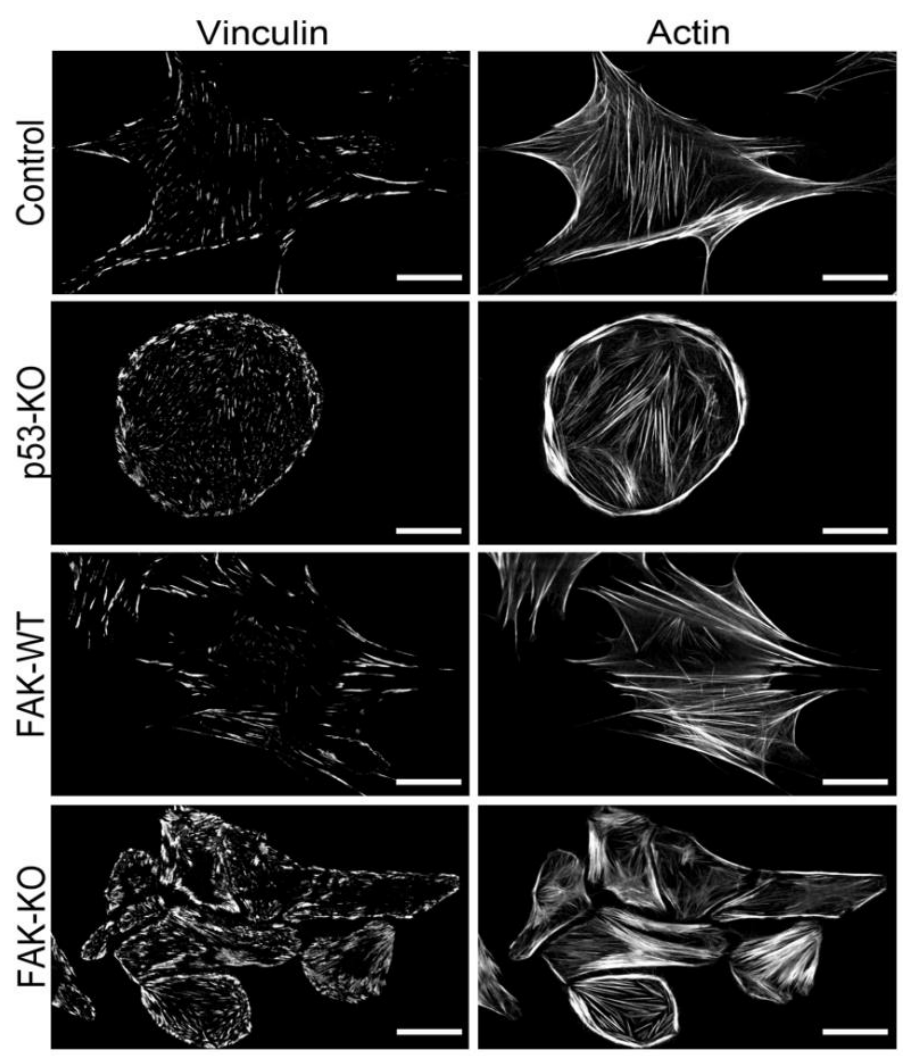

B

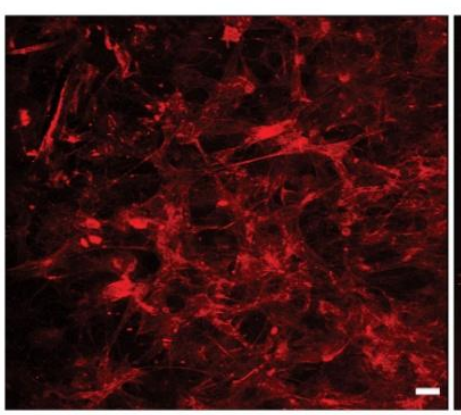

FAK-WT

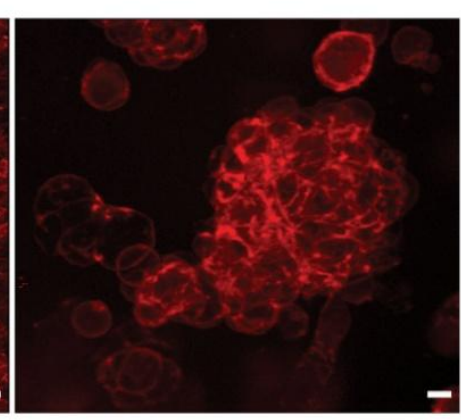

FAK-KO

C

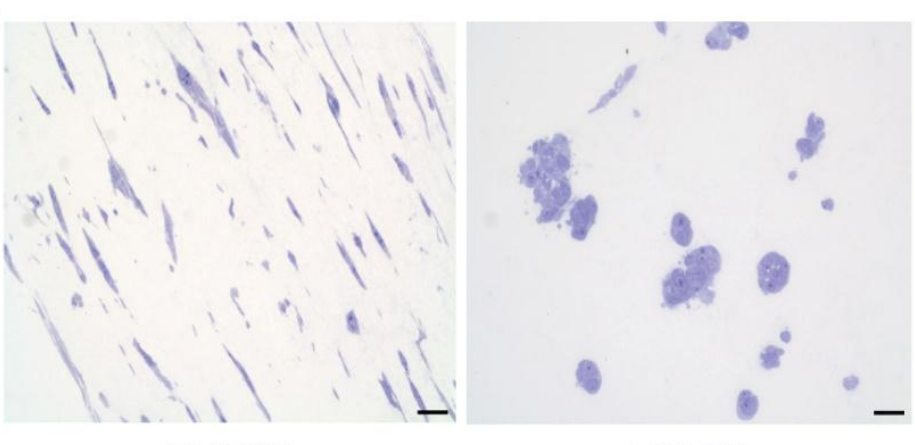

FAK-WT

FAK-KO

Figure 2 


\section{Figure 2: FAK knockout alters cell morphology in 2-D and 3-D.}

A) Preconfluent control, p53-KO, FAK-WT, and FAK-KO MEFs were grown for 24 hours on 25 $\mu \mathrm{g} / \mathrm{ml}$ collagen coated dishes. Cells were fixed, permeabilized, and stained with rhodamine phalloidin to visualize actin filaments or immunostained with monoclonal vinculin antibody to localize focal adhesions. Images were taken using a Zeiss 510 laser confocal microscope (63x/1.4 N.A. oil immersion objective). FAK-KO MEFs were small, round cells that do not spread with an increase in cortical actin and an increase in size and number of focal adhesions. Scale Bar $20 \mu \mathrm{m}$. (B) FAK-WT and FAK-KO collagen gels were stained with rhodamine phalloidin to visualize actin filaments. Images were taken using a two-photon fluorescent microscope (20x/0.4 N.A. objective). FAK-WT MEFs spread throughout the collagen gel forming an interconnected sheet of cells where as FAK-KO MEFs formed round cellular clusters throughout the depth of the gel. Scale Bar $20 \mu \mathrm{m}$. (C) FAK-WT and FAK-KO collagen gels were processed for electron microscopy, stained with Toluidine blue, and images were taken using the Olympus AX70 Provis microscope (40x/0.90 N.A. objective). Imaging showed thin, elongated FAK-WT cells that were interspersed in the gel where as FAK-KO MEFs were single, rounded cells that came together to form large cellular clusters. Scale Bar $20 \mu \mathrm{m}$. 


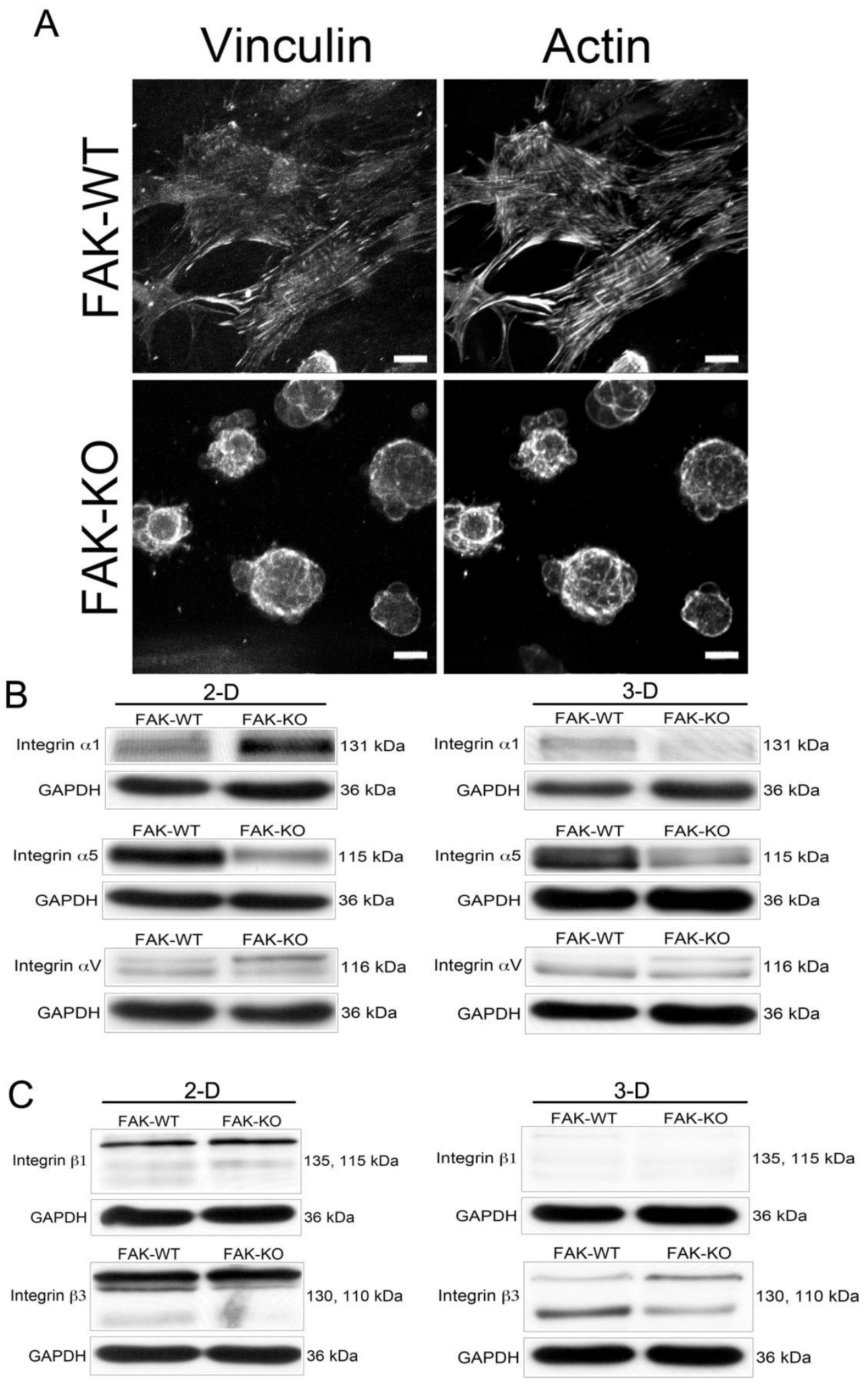

Figure 3 
Figure 3: Effect of the loss of FAK on 3-D focal adhesion distribution and integrin protein expression.

A) FAK-WT and FAK-KO collagen gels were stained with vinculin to visualize focal adhesions. Images were taken using a two-photon fluorescent microscope (40x/0.6 N.A. objective). FAKWT MEFs formed thin, elongated adhesions at the ends of the actin stress fibers. Vinculin in the FAK-KO MEFs accumulated within the cell, mainly around the periphery without forming focal adhesions. Scale Bar $20 \mu \mathrm{m}$. (B) FAK-WT and FAK-KO MEF 2-D and 3-D extracts were analyzed by Western blots for $\alpha_{1}$-integrin, $\alpha_{5}$-integrin, and $\alpha_{V}$-integrin protein levels. GAPDH was used as a loading control. (C) FAK-WT and FAK-KO MEF 2-D and 3-D extracts were analyzed by Western blots for $\beta_{1}$-integrin and $\beta_{3}$-integrin protein levels. GAPDH was used as a loading control. 


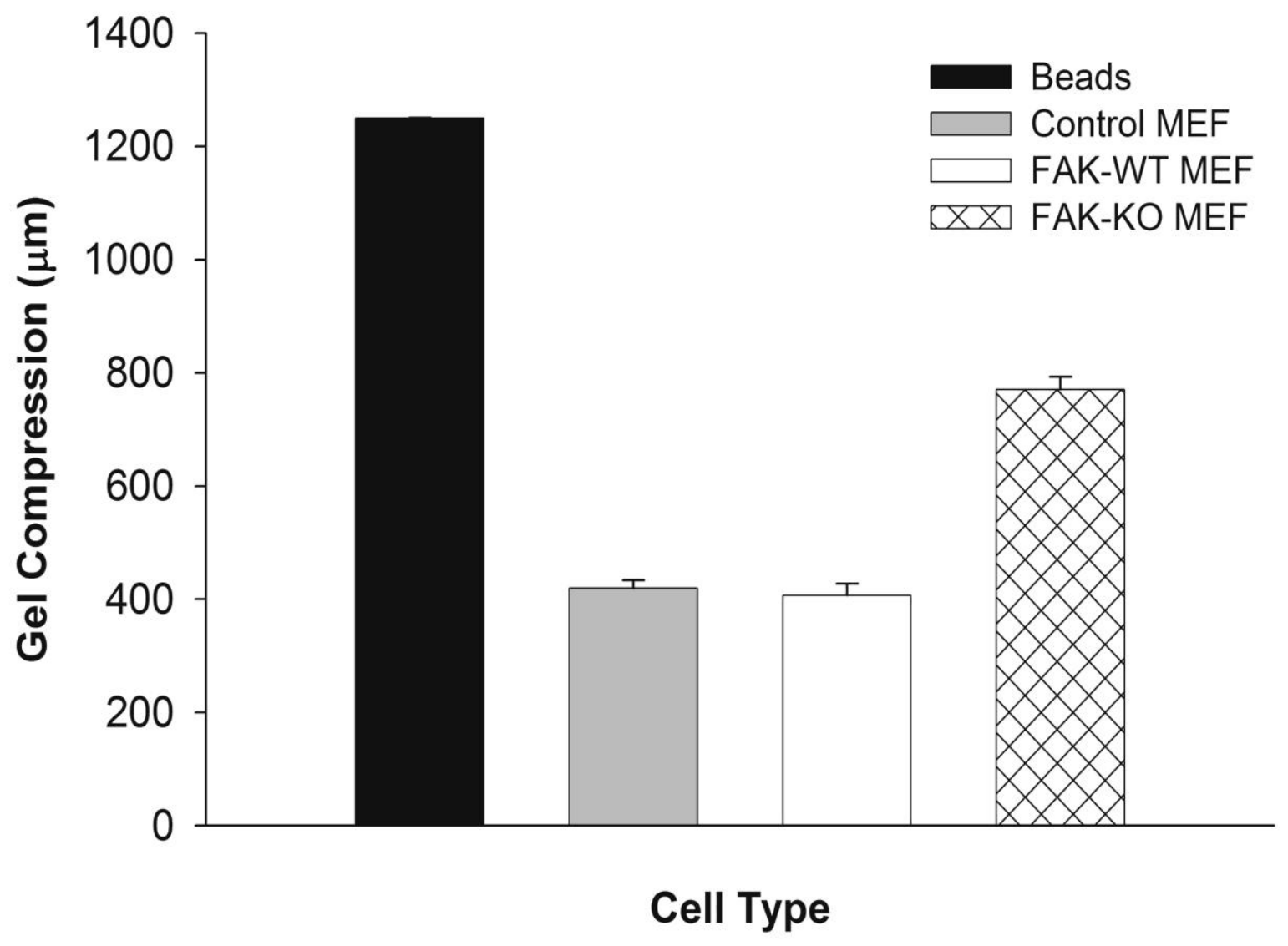

Figure 4 
Figure 4: Loss of FAK alters MEF ability to compress 3-D collagen gel.

Gel thickness of FAK-WT and FAK-KO MEF collagen gels were determined by staining the cells with CellTracker Red dye and determining the top and bottom of the gel using two-photon fluorescent microscopy (10x/0.3 NA objective). FAK-KO MEFs have the inability to compress the collagen gel. 


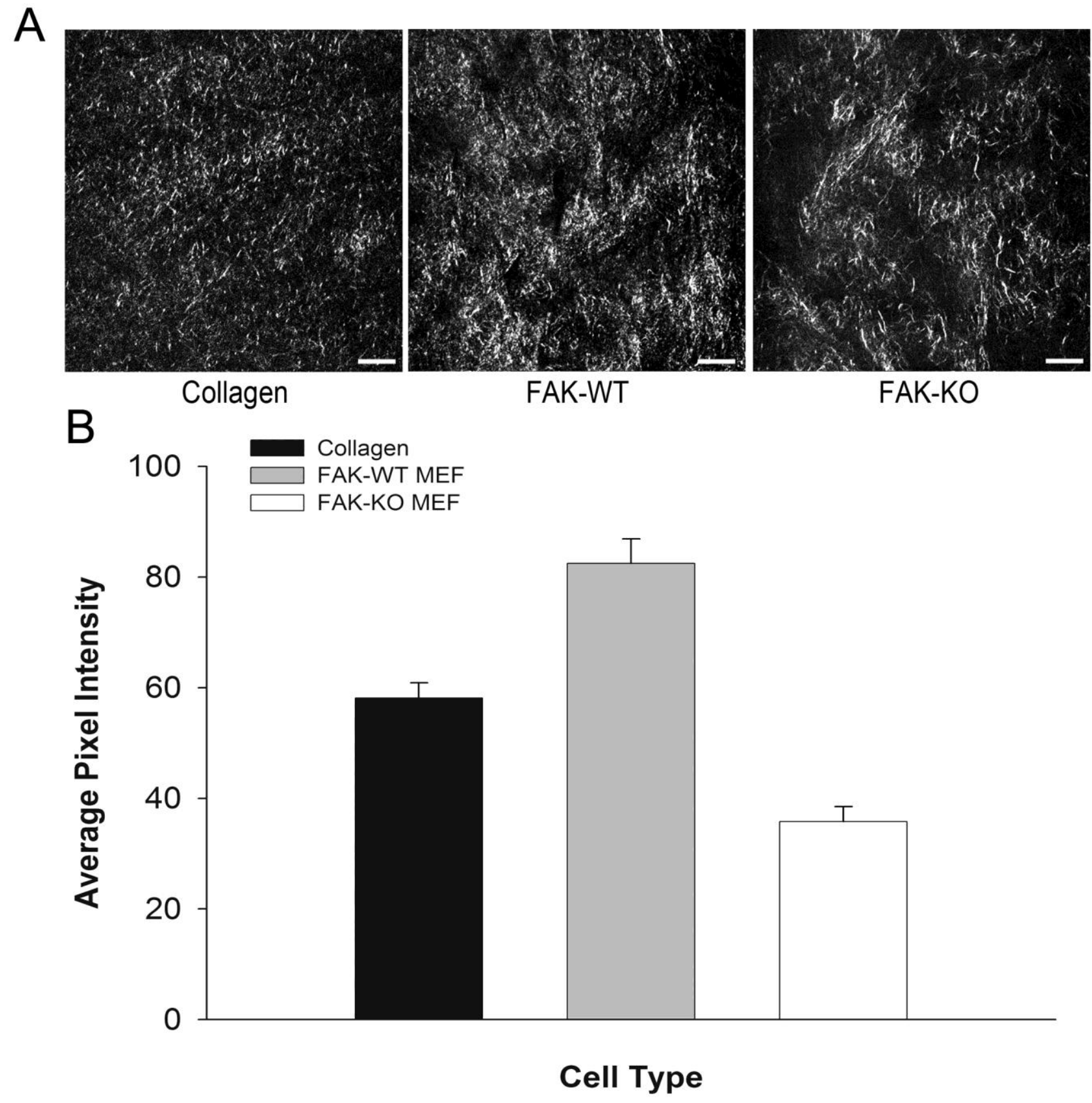

Figure 5 
Figure 5: Imaging of collagen matrix by second harmonic generation microscopy

A) Second harmonic generation ( $\mathrm{SHG}$ ) microscopy was utilized to image collagen matrix within collagen alone, FAK-WT, and FAK-KO MEF collagen gels (40x/0.8 water immersion objective). Loss of FAK resulted in low SHG signal much like a collagen gel devoid of cells. Scale Bar $20 \mu \mathrm{m}$. (B) Pixel intensity per image was determined using Fiji software. Data was presented as average pixel intensity per cell type. Error bars represent standard error of the mean. Each cell type had 10 images. Quantitation of pixel intensity revealed collagen within FAK-WT gels exhibited a stronger second harmonic signal compared to collagen alone and FAK-KO collagen gels. 


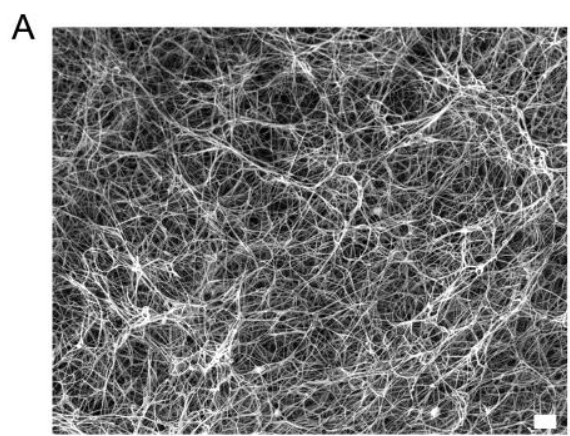

Collagen

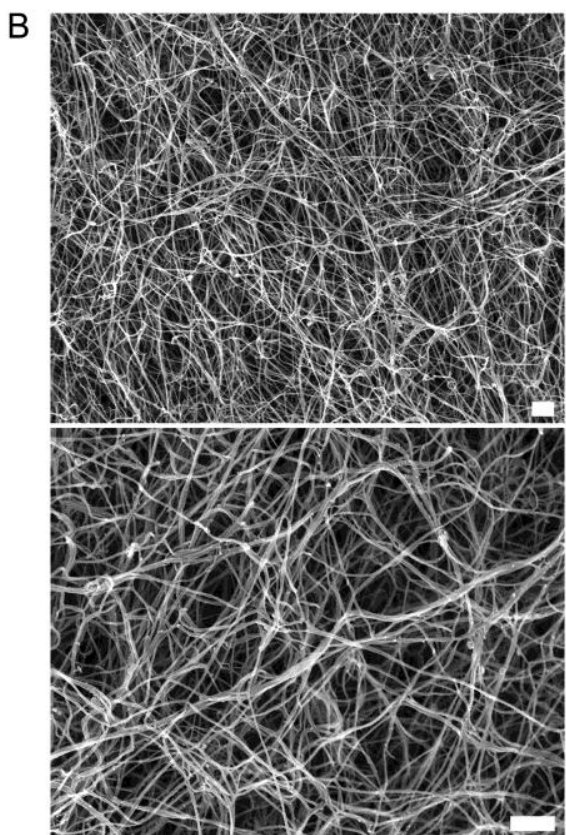

Collagen

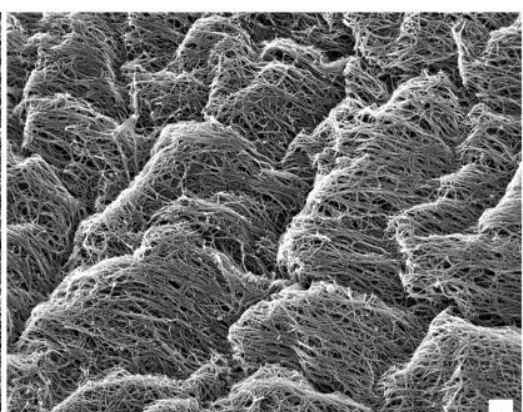

FAK-WT
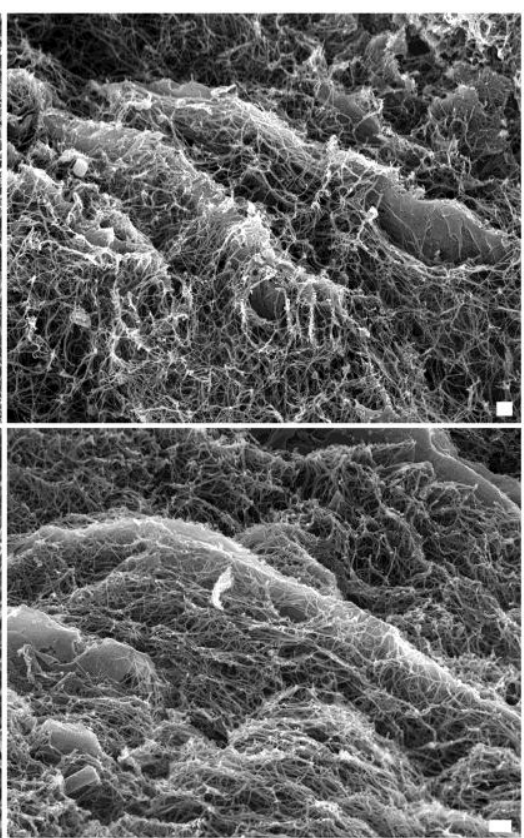

FAK-WT

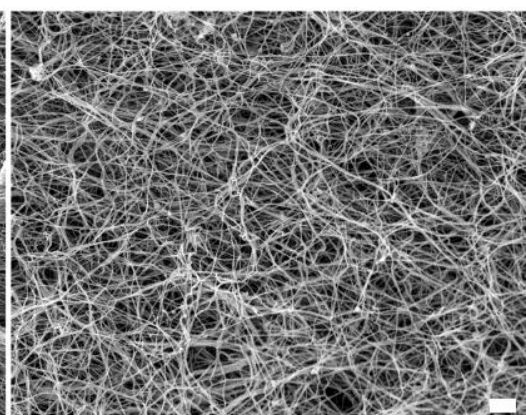

FAK-KO
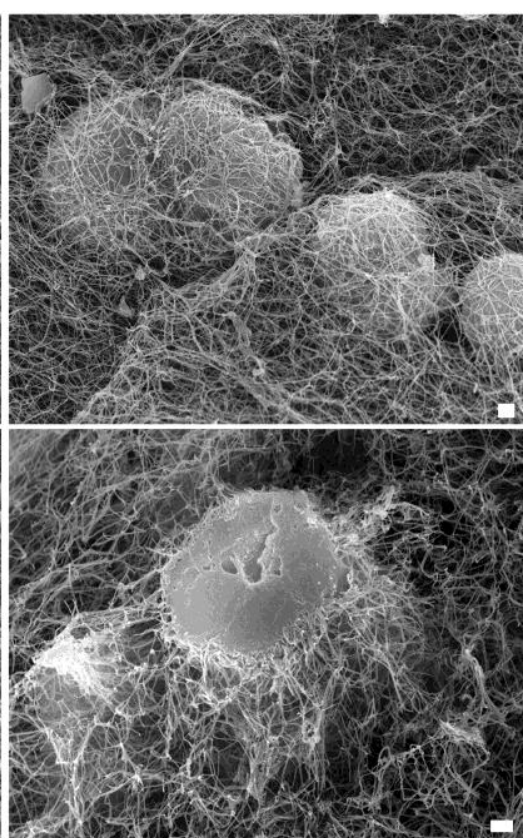

FAK-KO

Figure 6 


\section{Figure 6: Effect of the loss of FAK on 3-D collagen matrix organization}

A) The collagen matrix of FAK-WT and FAK-KO collagen gels were imaged using scanning electron microscopy. Collagen fibers in FAK-WT collagen gels formed a dense, organized matrix where as collagen in FAK-KO collagen gels formed a loose, unorganized matrix. Scale Bar $1 \mu \mathrm{m}$. (B) Freeze-fracture procedure was performed to visualize the collagen matrix deep within the collagen gel. Images were taken using a scanning electron microscope. Collagen fibers formed a dense network around the FAK-WT MEFs and completely engulfed the cells where as in the FAK-KO collagen gels, the collagen fibers were loosely arranged around the cellular clusters. Scale Bar $1 \mu \mathrm{m}$. 

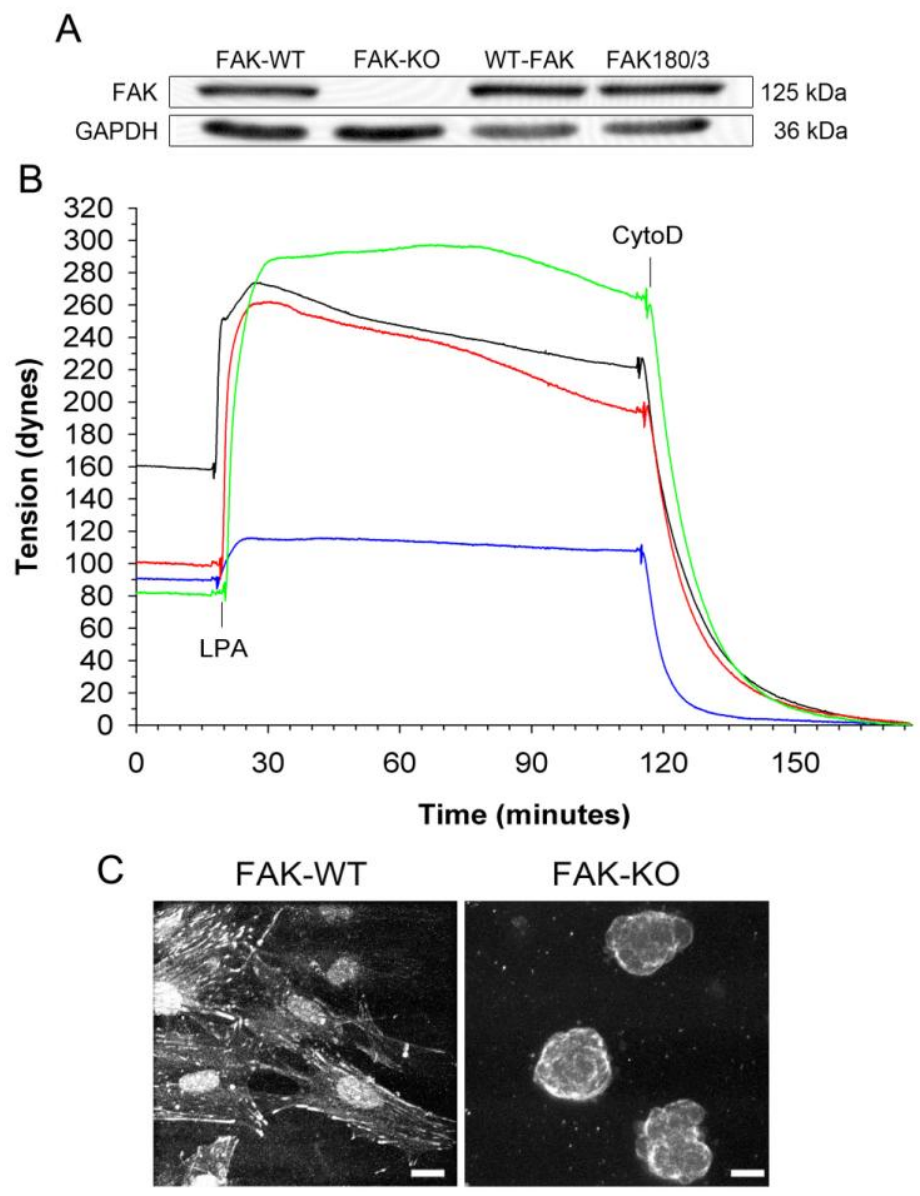

WT-FAK
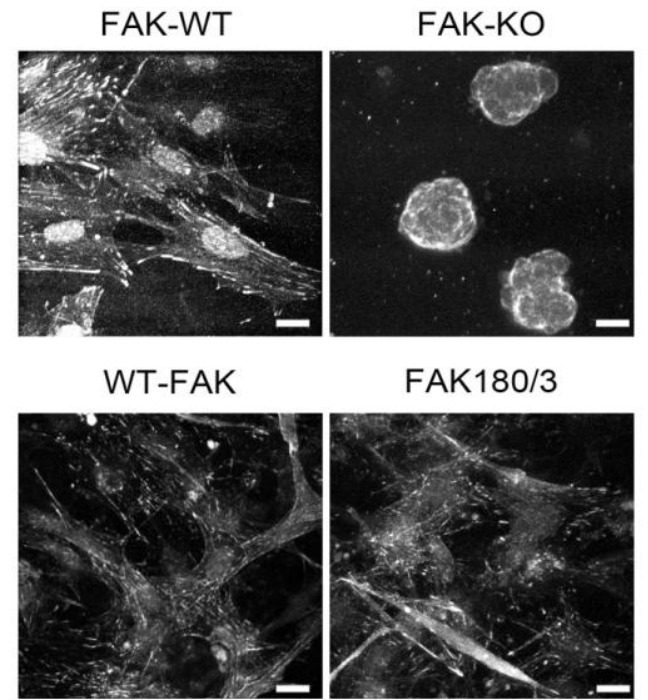

FAK180/3
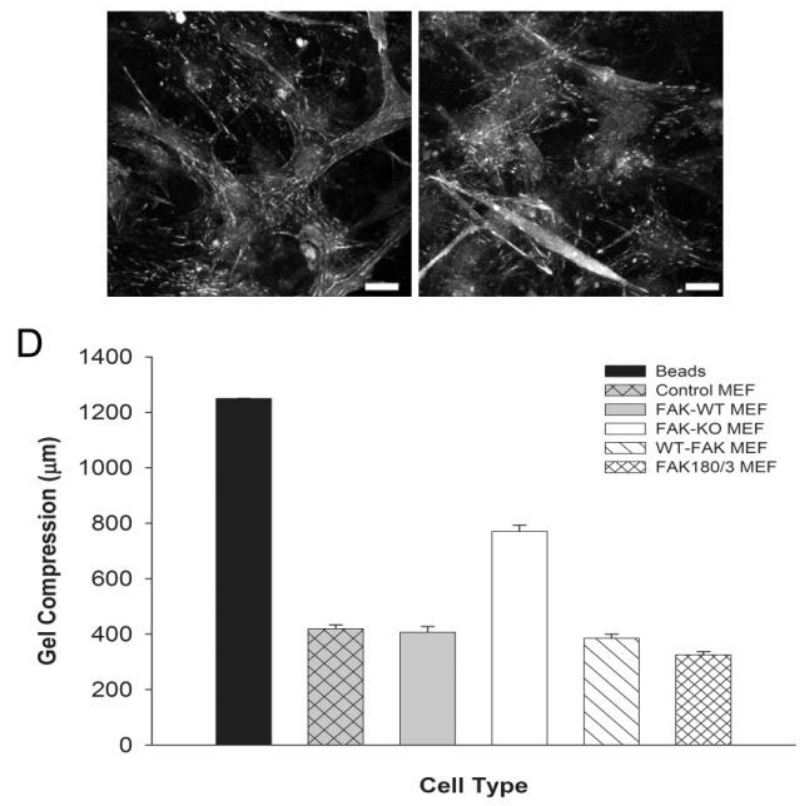

Figure 7 
Figure 7: Re-expression of FAK restores 3-D cell morphology, force generation, and gel compression.

FAK-KO MEFs were infected with either a lentivirus expressing a full length form of FAK (WT-FAK) or a constitutively active form of FAK (FAK180/3) to rescue FAK knockout. (A) Re-expression of FAK was determined in WT-FAK and FAK180/3 MEFs by Western blot analysis of FAK protein levels. GAPDH was used as a loading control. (B) Representative force tracing of tension produced by FAK-WT (black), FAK-KO (blue), WT-FAK (red), and FAK180/3 (green). Following establishment of basal tension, cells were stimulated with $1 \mu \mathrm{M}$ LPA. Re-expression of FAK restored agonist induced tension. (C) FAK-WT, FAK-KO, WTFAK, and FAK180/3 collagen gels were stained with vinculin to visualize focal adhesions. Images were taken using a two-photon fluorescent microscope (40x/0.6 N.A. objective). Both WT-FAK and FAK180/3 MEFs spread within the collagen gels and formed thin, elongated vinculin plaques at the end of actin stress fibers. Scale Bar $20 \mu \mathrm{m}$. (D) Gel thickness of FAKWT, FAK-KO, and re-expressing MEF collagen gels were determined by staining the cells with CellTracker Red dye and determining the top and bottom of the gel using two-photon fluorescent microscopy (10x/0.3 N.A. objective). Re-expression of FAK rescued the ability to compress the collagen gel. 


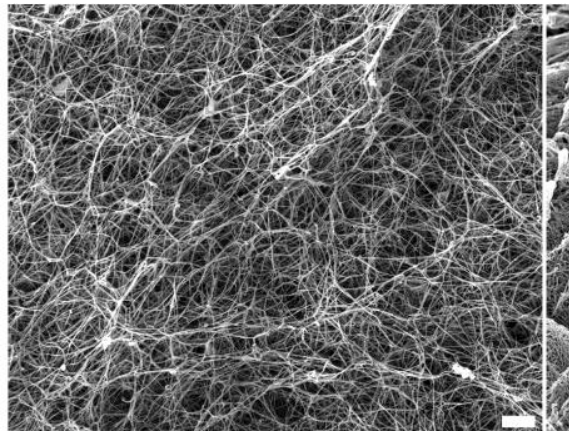

Collagen

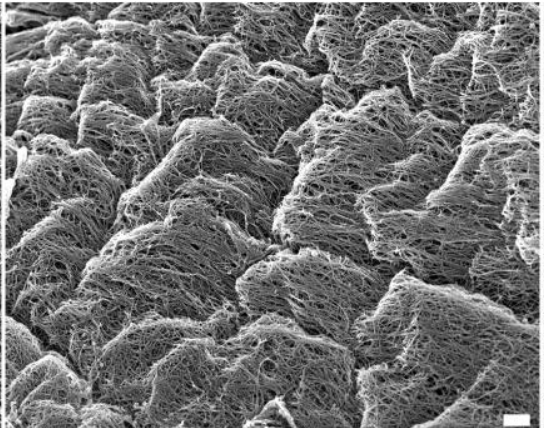

FAK-WT

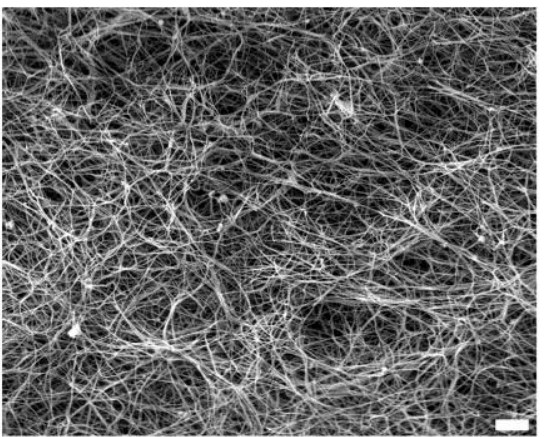

FAK-KO

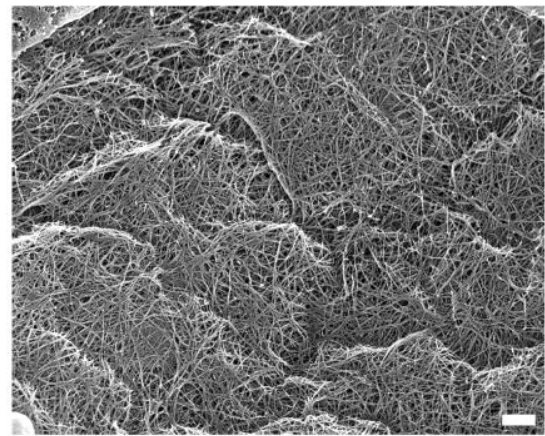

WT-FAK

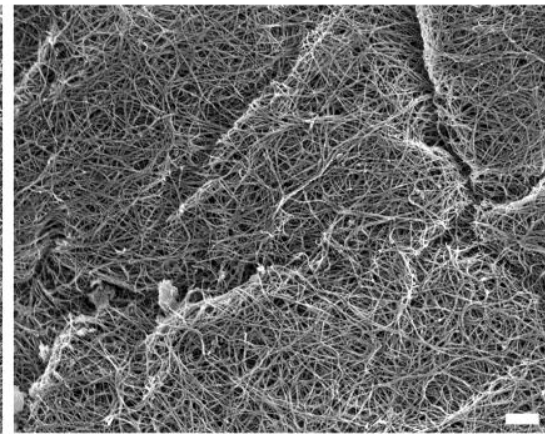

FAK180/3

\section{Figure 8}


Figure 8: Re-expression of FAK restores collagen matrix organization.

The collagen matrix of FAK-WT, FAK-KO, WT-FAK, and FAK180/3 collagen gels were imaged using scanning electron microscopy. Collagen fibers in WT-FAK and FAK180/3 formed a dense, organized matrix like in the FAK-WT collagen gels. Re-expression of FAK in FAKKO MEFs restored the ability to organize the collagen matrix. Scale Bar $1 \mu \mathrm{m}$. 

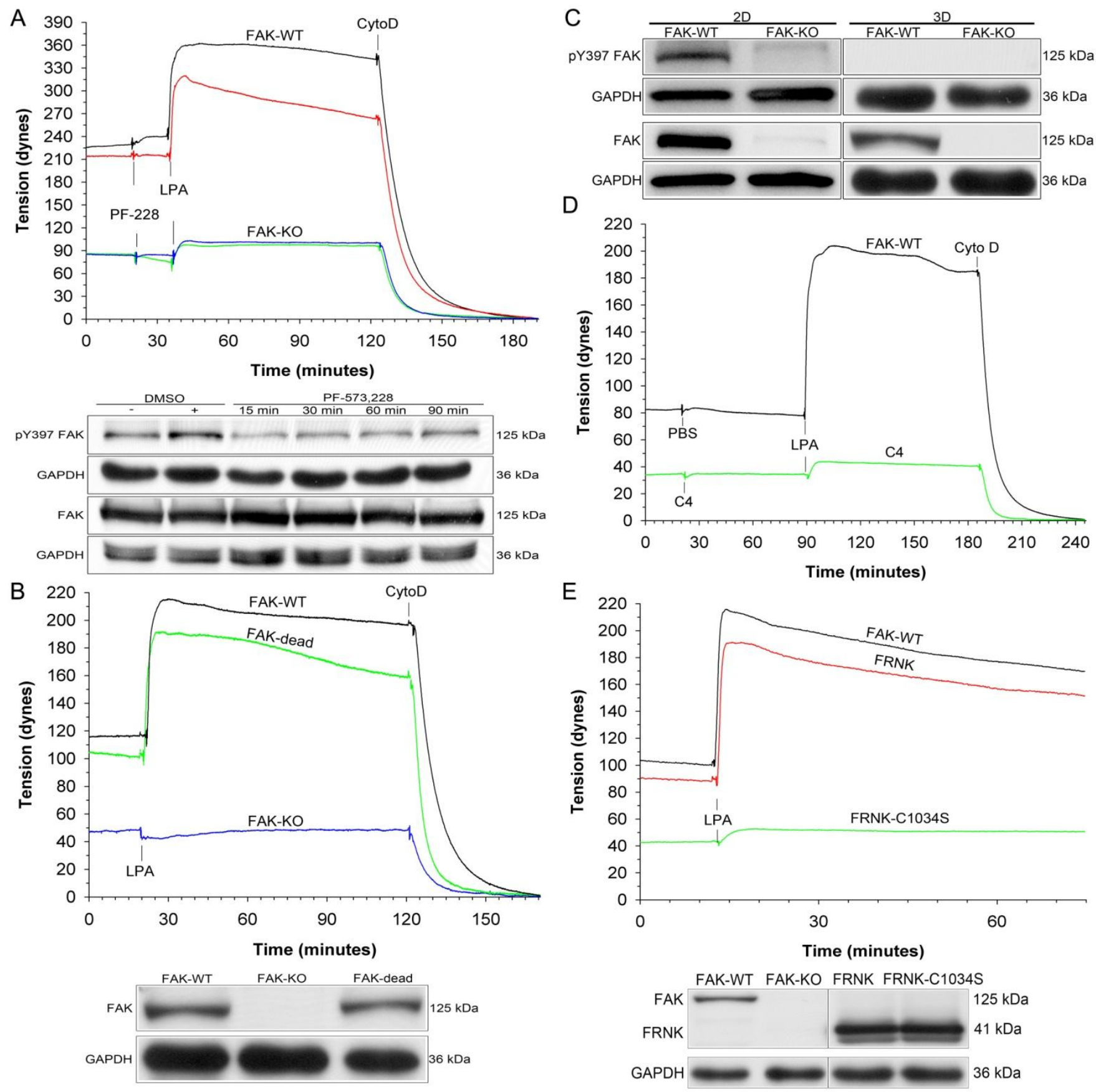

Figure 9 
Figure 9: FAK localization in focal adhesions and not kinase activity is important for tension generation

A) Representative force tracing of tension produced by FAK-WT treated with DMSO (black) or PF-573,288 (red) and FAK-KO MEFs treated with DMSO (blue) or PF-573,288 (green). Following establishment of basal tension, cells were treated with DMSO or $1 \mu \mathrm{M}$ PF-573,228 for 15 minutes and then stimulated with $1 \mu \mathrm{M}$ LPA. Inhibition of FAK kinase activity did not alter basal or agonist induced force. Time-course studies were performed on FAK-WT MEFs to determine greatest inhibition of FAK kinase activity. Level of phosphorylated tyrosine residue 397 of FAK was analyzed by Western blot to determine activation of FAK. GAPDH was used as a loading control. (B) Representative force tracing of tension produced by FAK-WT (black), FAK-KO (blue), and a kinase dead mutant form of FAK expressed in FAK-KO MEFs (FAKdead) (green). FAK-dead MEFs had a similar basal and agonist induced force as FAK-WT MEFs. Following tension experiments, expression of FAK was determined by Western blot. GAPDH was used as a loading control. (C) FAK-WT and FAK-KO MEF 2-D and 3-D extracts were analyzed for phosphorylated FAK protein levels by Western blots. GAPDH was used as a loading control. There was a robust amount of phosphorylated FAK in 2-D however FAK was not phosphorylated in 3-D. (D) Representative force tracing of tension produced by FAK-WT (black) and chloropyramine hydrochloride (C4) treated FAK-WT (green). Following stable basal tension, cells were treated with $1 \mu \mathrm{M} \mathrm{C} 4$ followed by stimulation with $1 \mu \mathrm{M}$ LPA. MEFs treated with $\mathrm{C} 4$ produced a lower basal tension than FAK-WT MEFs and minimal agonist induced force. (E) Representative force tracing of tension produced by FAK-WT (black), FAKrelated non-kinase (FRNK) expressed in FAK-KO MEFs (red), and a mutant form of FRNK (FRNK-C1034S) that does not localize to focal adhesions (green). FRNK MEFs had a similar 
basal and agonist induced force as FAK-WT MEFs where as FRNK-C1034S MEFs have low basal tension and minimal agonist induced force. Following tension experiments, expression of FRNK was determined by Western blot. GAPDH was used as a loading control. 


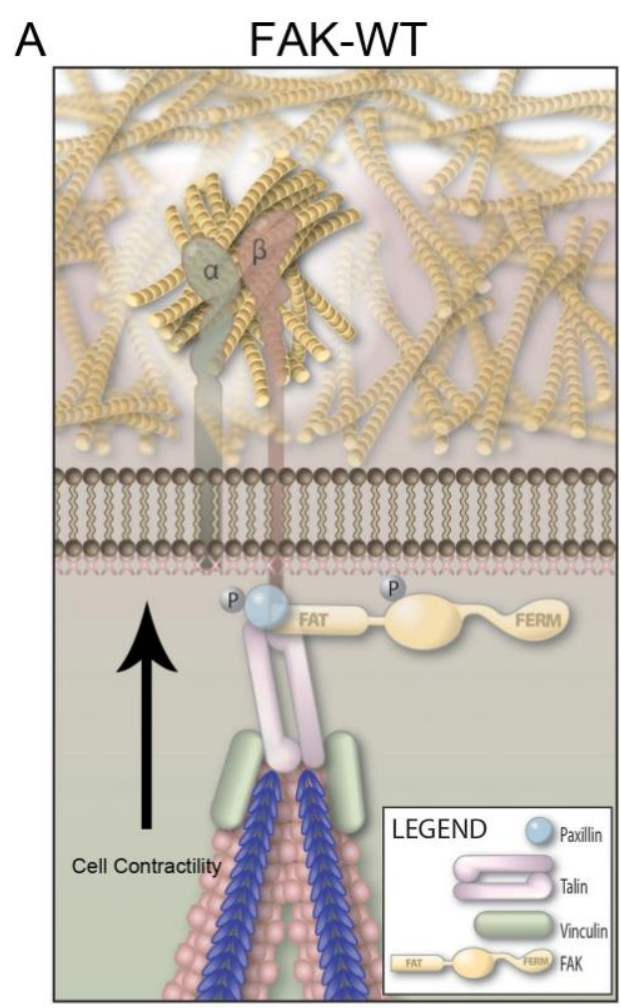

B

FAK-KO

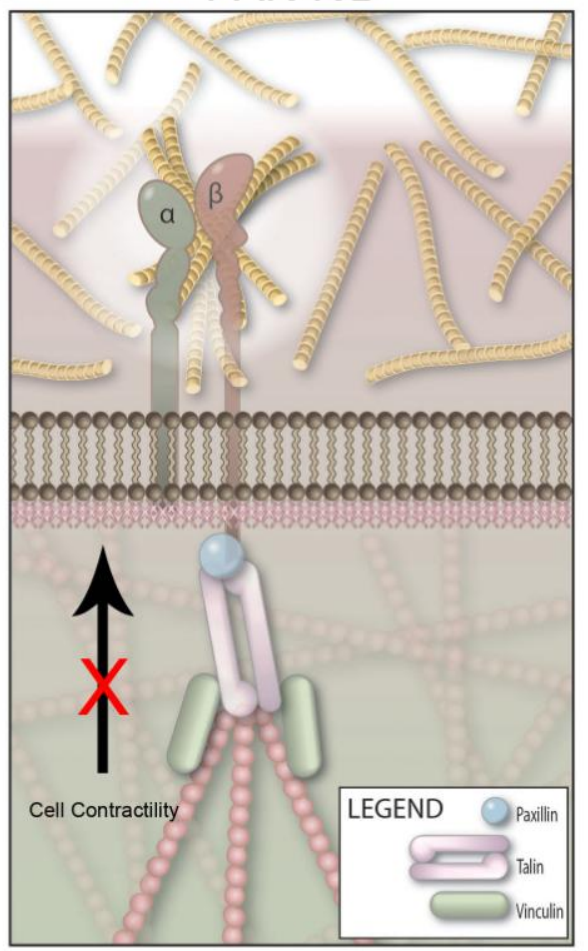

C

FRNK

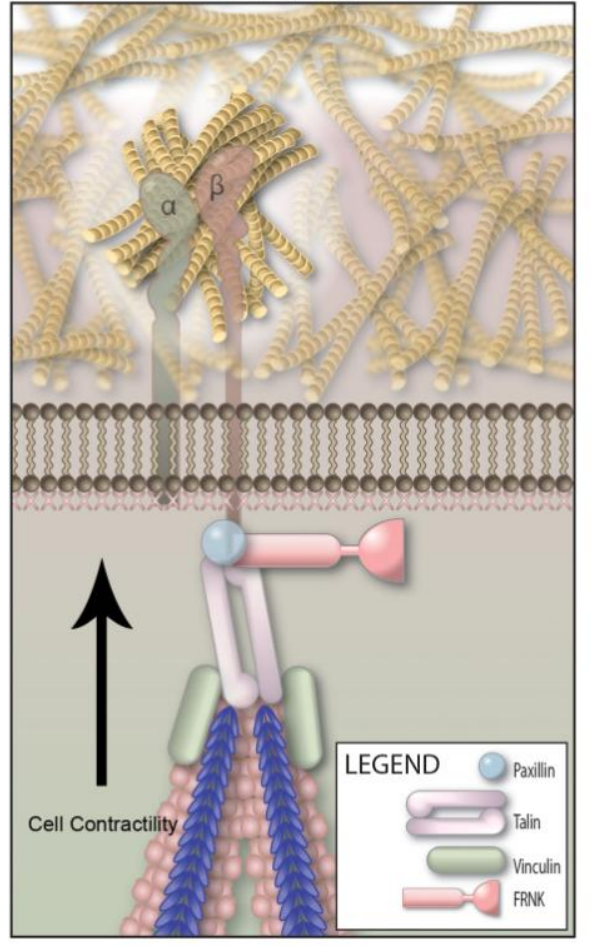

D

FRNK-C1034S

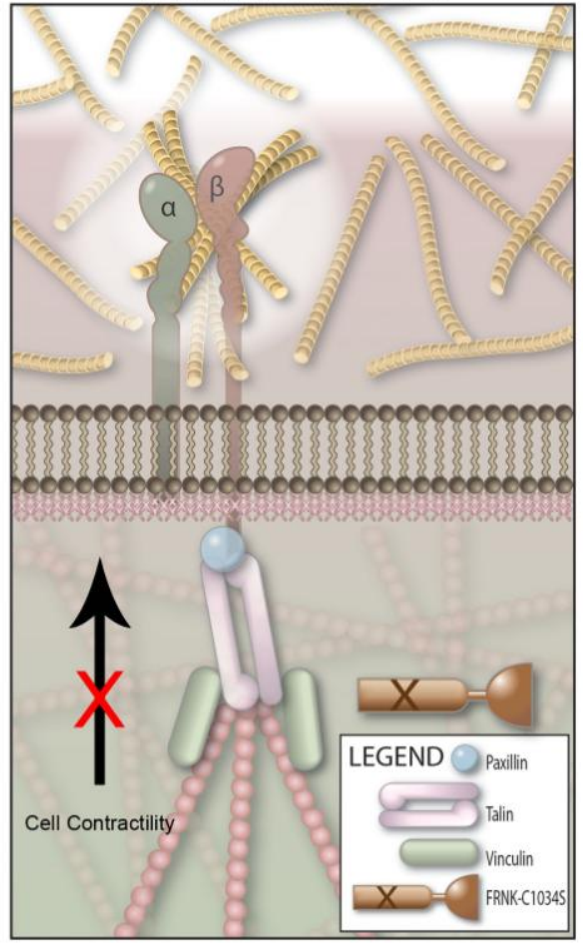

Figure 10 
Figure 10: Mechanism of FAK regulation of fibroblast tension generation and collagen matrix organization

A) FAK is localized to the focal adhesions and is necessary for the proper arrangement of the adhesion proteins and linkage to the actin cytoskeleton to allow internal force to be transmitted through the adhesion to the collagen matrix. Transmission of force to the ECM results in organization of the collagen fibers into a dense, organized network. This anchors the cell to the substrate, allowing for cell contraction to occur. B) Knockout of FAK causes an accumulation of focal adhesion proteins and an increase in cortical actin without classical focal adhesion formation. The result is a weak link between the actin cytoskeleton and focal adhesions. An increase in internal tension occurs, however due to the actin cytoskeleton not being linked up properly, force cannot be transmitted through the adhesion to the collagen matrix, resulting in the inability to organize the collagen fibers. C) FRNK localizes to the focal adhesions and this results in a phenotype similar to FAK-WT MEFs with the formation of a dense network of collagen fibers and the induction of cell contraction. D) Expression of a mutant form of FRNK that does not localize to focal adhesions (FRNK-C1034S) has a similar phenotype to the FAKKO MEFs with a lack of collagen matrix organization and minimal force generation, suggesting that FAK localization to the focal adhesion is critical for cell contraction and matrix organization. 
Supplementary Figures
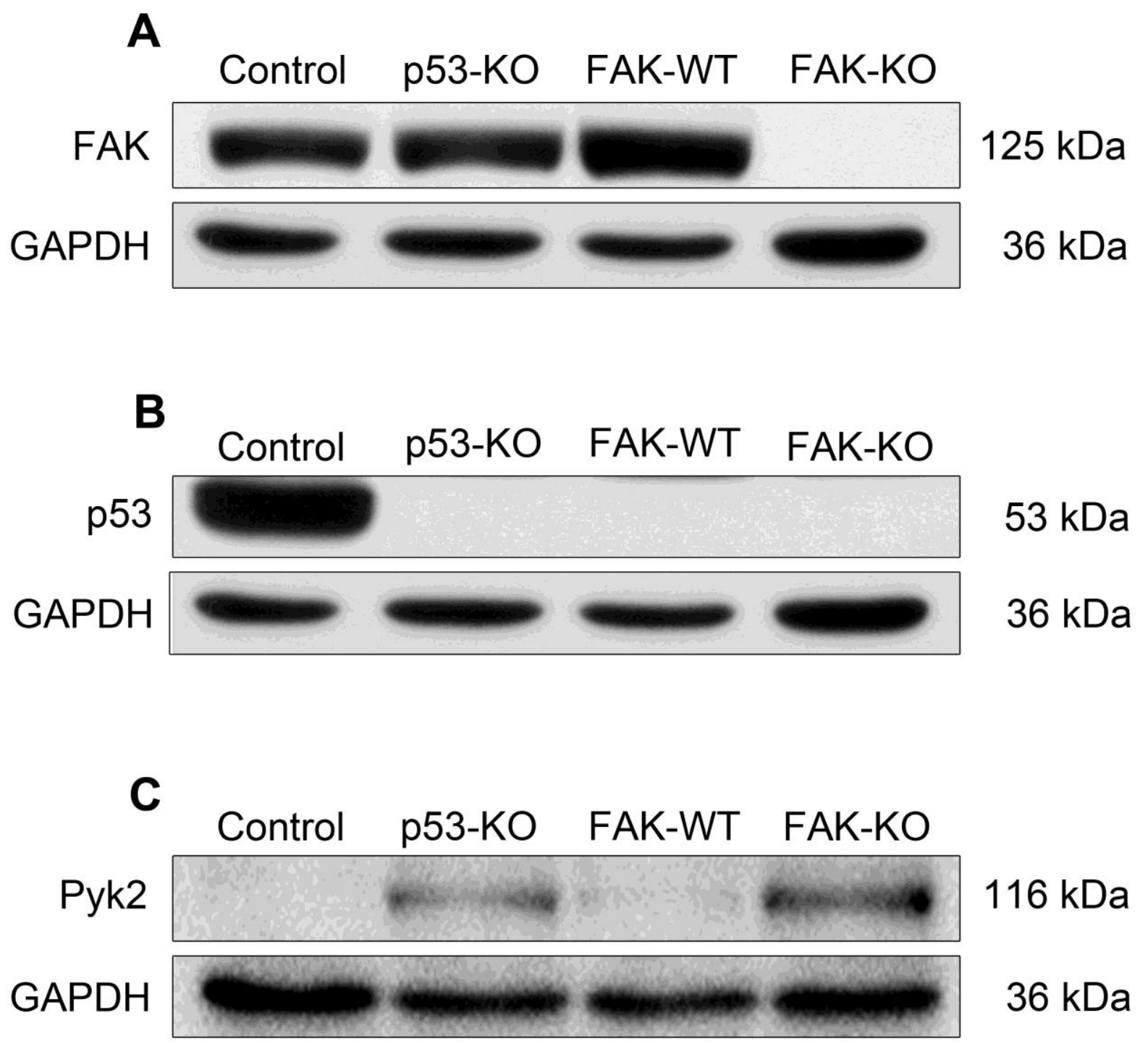

Supplementary Figure 1 
Supplementary Figure 1: Analysis of FAK, Pyk2 and p53 protein levels.

WT, p53-KO, FAK-WT, and FAK-KO MEF extracts from 3-D collagen gels were assessed for (A) FAK, (B) Pyk2, and (C) p53 protein levels. GAPDH was used as a loading control. 


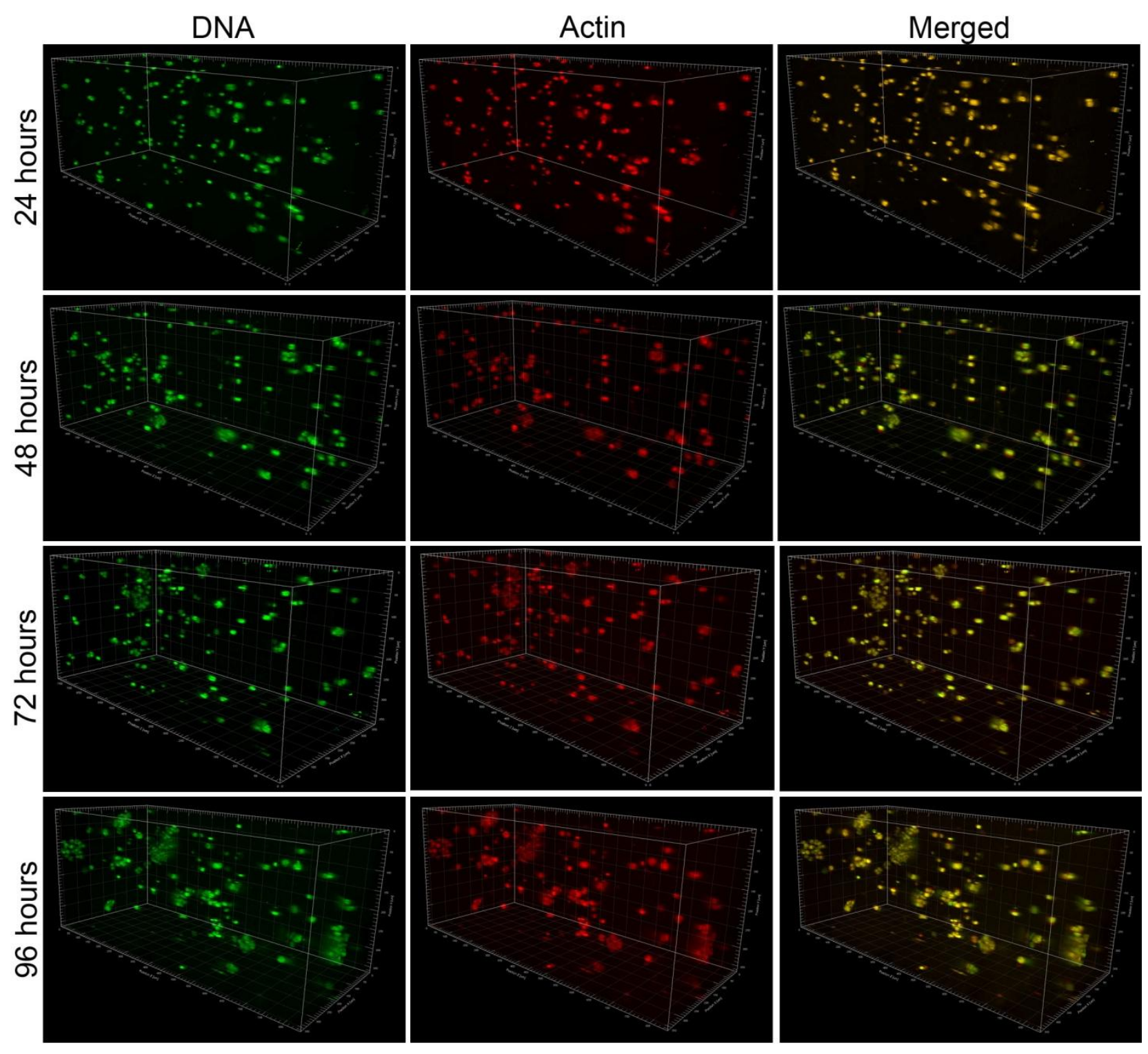

Supplementary Figure 2 


\section{Supplementary Figure 2: DNA staining}

FAK-KO MEFs cell nuclei and actin filaments were stained day 1, 2, 3 or 4 post-seeding to determine cell distribution within the collagen gel. Cells were imaged using two-photon fluorescent microscopy (20x/0.4 N.A. objective). On day 1, FAK-KO MEFs were evenly distributed along the depth of the collagen gel. Over the course of 4 days, the FAK-KO MEFs clustered together forming large conglomerates of cells throughout the depth of the gel. 

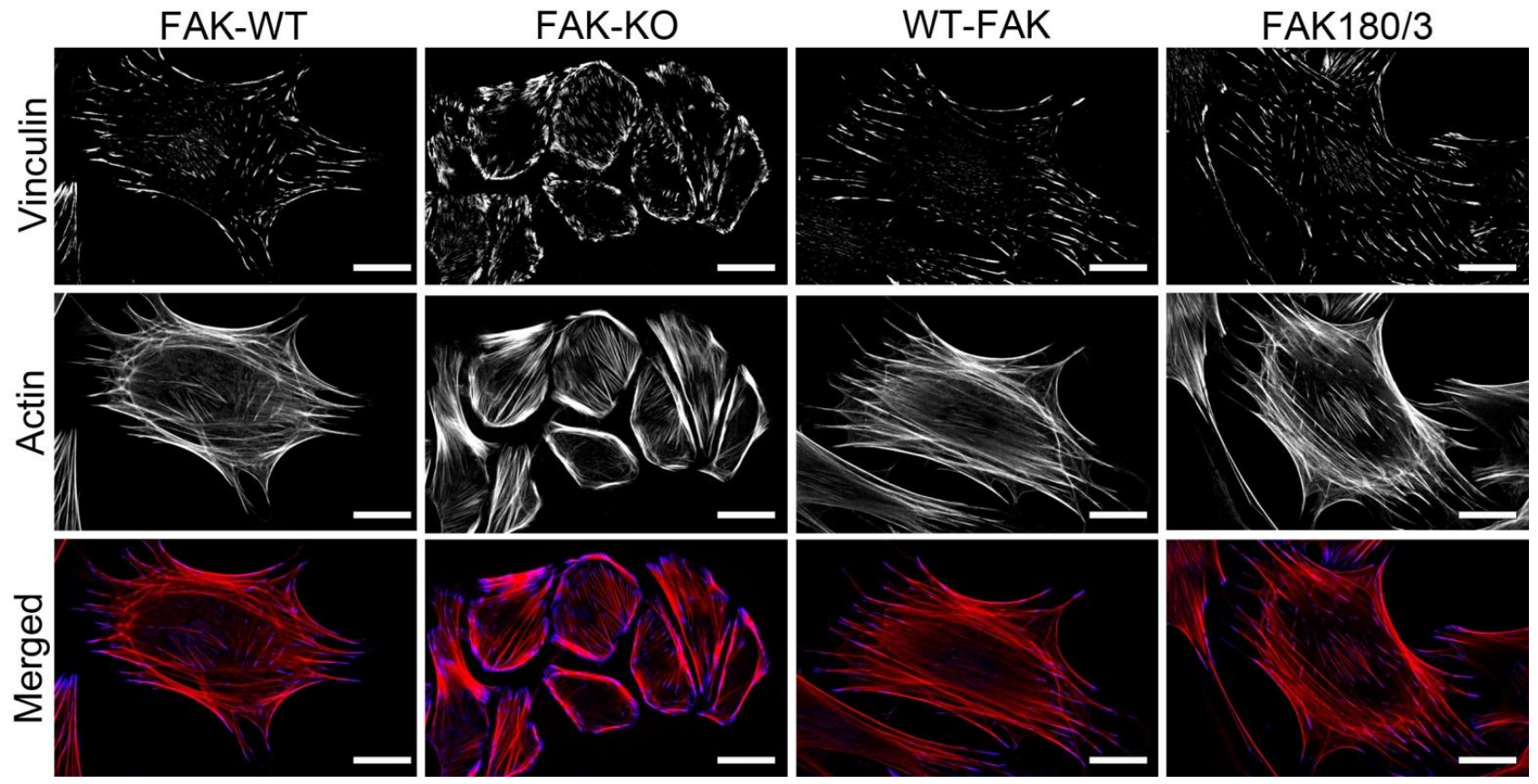

Supplementary Figure 3 
Supplementary Figure 3: Re-expression of FAK restores cell spreading and focal adhesion formation in 2-D.

Preconfluent FAK-WT, FAK-KO, WT-FAK, and FAK180/3 MEFs were grown for 24 hours on $25 \mu \mathrm{g} / \mathrm{ml}$ collagen coated dishes. Cells were fixed, permeabilized, and stained with rhodamine phalloidin to visualize actin filaments or immunostained with monoclonal vinculin antibody to localize focal adhesions. Images were taken using a Zeiss 510 laser confocal microscope (63x/1.4 N.A. oil immersion objective). Re-expression of FAK restored cell spreading and focal adhesion distribution in 2-D. Scale Bar $20 \mu \mathrm{m}$. 

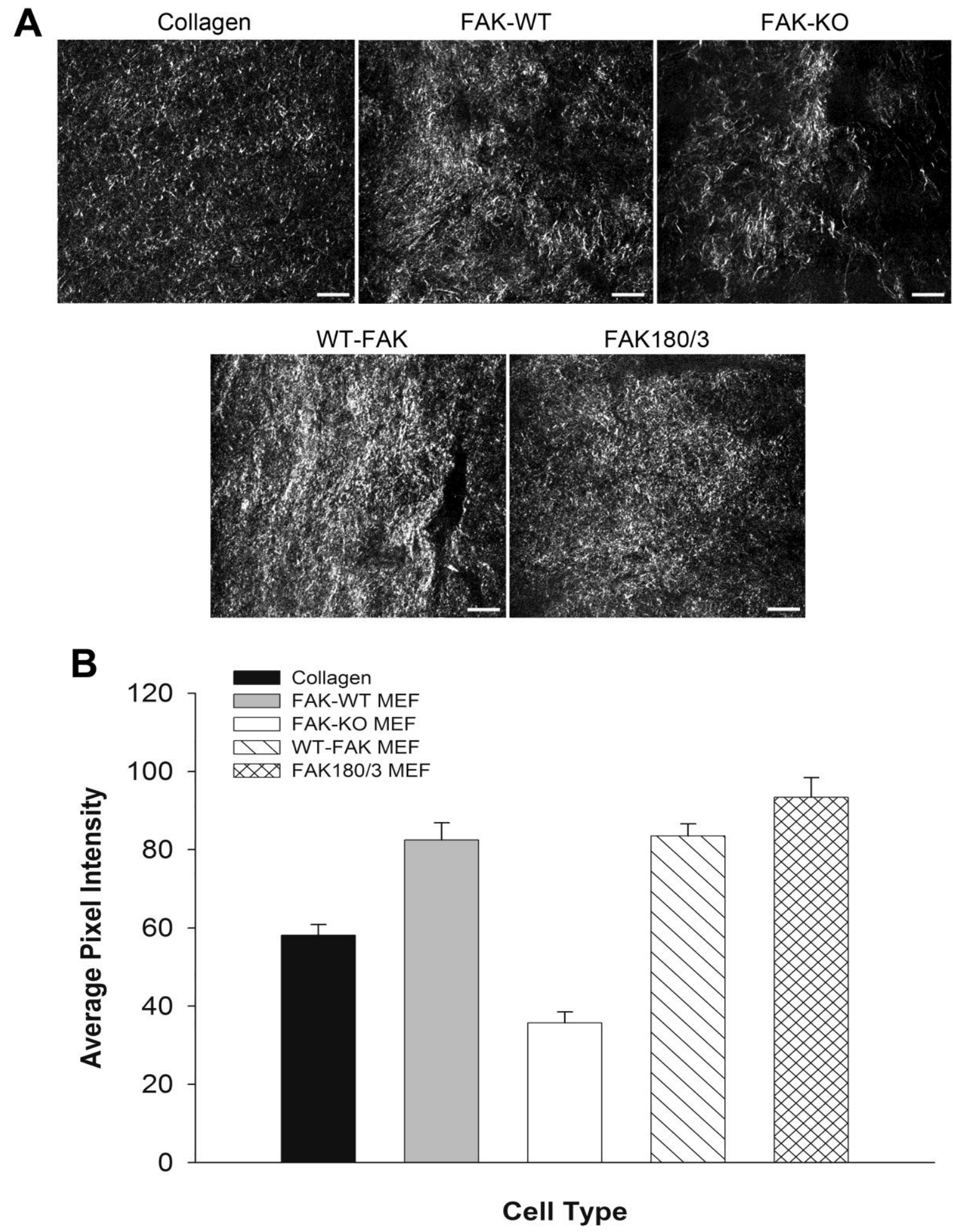

Supplementary Figure 4 


\section{Supplementary Figure 4: Restoration of SHG signal in FAK re-expressing MEFs}

A) Second harmonic generation ( $\mathrm{SHG}$ ) microscopy was utilized to image collagen matrix within collagen alone, FAK-WT, FAK-KO, WT-FAK, and FAK180/3 MEF collagen gels (40x/0.8 N.A. water immersion objective). Scale Bar $20 \mu \mathrm{m}$. (B) Pixel intensity per image was determined using Fiji software. Data was presented as average pixel intensity per cell type. Error bars represent standard error of the mean. Each cell type had 10 images. Quantitation of pixel intensity revealed both WT-FAK and FAK180/3 produced a similar strong SHG signal as FAKWT MEFs. 


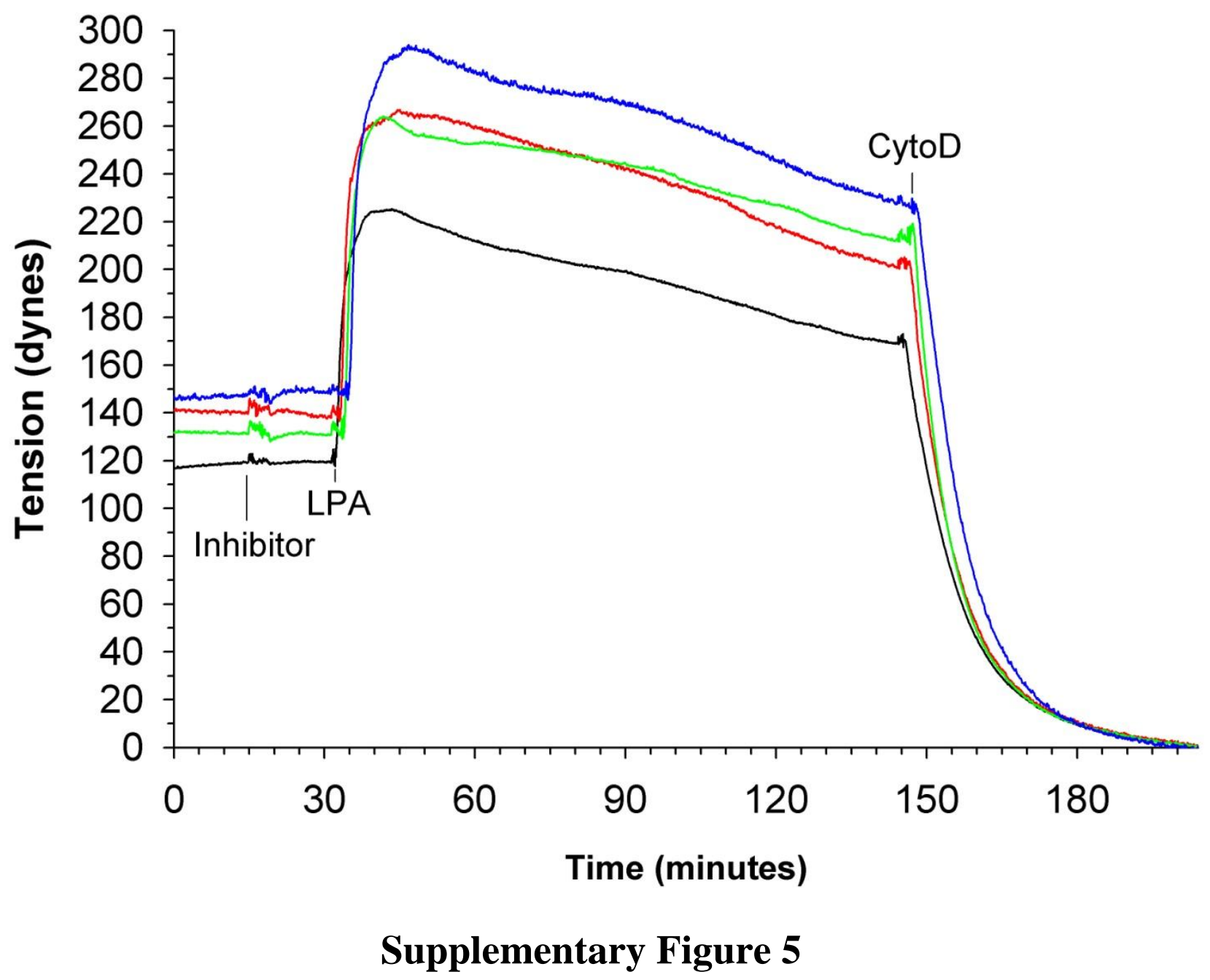




\section{Supplementary Figure 5: Inhibition of Src-family of kinases does not affect force generation}

FAK-WT MEFs were treated with water (black), DMSO (red) or $1 \mu \mathrm{M}$ of the Src-family kinase inhibitors PP2 (green) or SU6656 (blue) for 15 minutes prior to stimulation with LPA. Inhibition of the activity of the Src-family kinases did not alter FAK-WT basal and agonist-induced tension generation. 


\section{CHAPTER 4:}

\section{General Discussion}


FAK was initially discovered to be a protein crucial in transducing signals following integrin engagement to influence cellular behavior (Guan et al., 1991; Kornberg et al., 1991; Guan and Shalloway, 1992; Hanks et al., 1992; Kornberg et al., 1992; Schaller et al., 1992). Over the past 20 years, FAK has been implicated in having a role in a vast array of processes that includes cell motility and survival, to being a key factor in the development of diseases such as cardiovascular complications, fibrosis, and cancer (Ilic et al., 1995; Sieg et al., 1999; Sieg et al., 2000; Gabarra-Niecko et al., 2003; van Nimwegen, et al., 2005; Clemente et al., 2007; Peng et al., 2008; Wong et al., 2011; Lagares et al., 2012). Due to its location in the focal adhesions and connection with the contractile cytoskeleton and ECM, FAK has become a prime candidate for the cell's ability to sense and generate mechanical forces. However, current literature is not clear as to the role of FAK in tension generation. Also, studies primarily focus on investigating the role of FAK in cells seeded on a 2-D substrate coated surface. In the body, interactions between cells do not exist in a singular plane but in all directions. Therefore, it is important to study cell behavior in a system that closely mimics the physiological condition.

The overall goal of this dissertation was to gain a better understanding of how FAK is involved in the generation and transmission of nonmuscle cell contraction. In order to investigate this, tension of FAK knockdown endothelial cells and FAK knockout fibroblasts was measured in a 3-D collagen matrix. Knockdown of FAK in human pulmonary microvessel endothelial cells altered both cell morphology and actin distribution, and increased focal adhesion formation. Measuring tension generation in 3-D reveled that FAK depleted endothelial cells developed a higher basal tension without alterations in myosin II phosphorylation. However, agonist induced tension generation was not altered in FAK knockdown endothelial cells. The loss of FAK caused an increase in monolayer barrier function, which coincided with a 
slight alteration in VE-cadherin localization. Therefore, the loss of FAK in endothelial cells resulted in rearrangement of the actin cytoskeleton and enhancement in cell attachment to the underlying matrix producing a greater basal cellular tension, resulting in alterations in cell-cell cohesion and the formation of a tighter monolayer. However, measurement of tension generation by FAK knockout fibroblasts revealed that the loss of FAK reduced basal force and produced minimal agonist induced force generation without alterations in myosin II phosphorylation. The lack of classical focal adhesion formation and inability to organize the collagen matrix in the absence of FAK in 3-D suggests that FAK is responsible in the stabilization of the focal adhesions and transmission of force through the focal adhesion to the ECM in order to organize the collagen fibers and result in cell contraction. Inhibition of FAK kinase activity showed that FAK functions in a kinase independent manner to regulate force generation.

It was observed that loss of FAK resulted in an increase in basal force in endothelial cells but caused a reduction in basal force and produced minimal agonist induced force in fibroblasts. The reason for this difference in response to the loss of FAK is two-fold. FAK may play differing roles in each cell type depending on the individual cell's physiological function. Endothelial cells line the inner wall of blood vessels and regulate the movement of solutes across the vessel wall, as well as aiding in control of vascular dilation and constriction. Fibroblasts are among the most common cells in connective tissue and are involved in synthesizing the ECM proteins and play a critical role in wound healing. It has been shown that endothelial cells derived from different vascular beds exhibit differences in permeability suggesting that one has to consider not only the cell type but the location that the cells were isolated from (Schnitzer et 
al., 1994). Also, there may be differences in FAK function between species since the endothelial cells were of human origin and the fibroblasts were isolated from mice.

Secondly, the modulation of FAK protein levels was achieved in two different ways, knockdown with shRNA targeting FAK mRNA in endothelial cells and a genetic knockout in fibroblasts. Due to the nature of shRNA targeting of proteins, there was still at least $10 \%$ of the FAK protein remaining in the endothelial cells. This may be enough protein present to lock the focal adhesions to the actin cytoskeleton and allow the strengthening of the adhesion in order to transduce the cellular force to the ECM and allow cell contraction. Complete loss of FAK in fibroblasts resulted in a reduction in basal force and minimal production of agonist induced force. Current investigations are underway in our lab to create a stable FAK knockdown human dermal fibroblast cell line to investigate how knockdown of FAK alters tension generation in fibroblasts and see if it results in a similar phenotype exhibited by FAK knockout fibroblasts.

Our knowledge regarding FAK's role in cell contractility is derived primarily from studies conducted on cells seeded onto 2-D tissue culture substrates. However, studies have shown differences in cell morphology, biochemical signaling, and function in 2-D versus 3-D culture. Roskelley and Bissell (1995) have shown that epithelial cells grown in a 3-D environment promote normal epithelial polarity and differentiation compared to 2-D. Fibroblasts are normally nonpolar cells, however, culturing them on 2-D surfaces induces an artificial polarity. When fibroblasts are suspended in collagen or seeded onto tissue-derived 3-D matrix, they exhibit a nonpolar morphology and enhanced adhesion and cell migration (Elsdale and Bard, 1972; Friedl and Brocker, 2000; Cukierman et al., 2001). The physical structure of the 3D matrix appears to provide information that is not present when cells are attached to a ligand coated surface altering biochemical signaling and cell function. It is also uncertain if there is 
formation of stress fibers and discrete focal adhesions in 3-D. When cells are completely within a 3-D matrix in vivo or when placed on soft substrates, focal adhesions are not readily detectable (Friedl et al., 1998; Knight et al., 2000; Petroll et al., 2003). Utilization of live cell microscopy of cells embedded in a collagen matrix reveals no aggregation of common focal adhesion proteins (Fraley et al., 2010) where as other studies utilizing tagged focal adhesion proteins show focal adhesion formation (Kubow and Horwitz, 2011). However, it has been shown that the molecular composition of the focal adhesions differs in 3-D (Cukierman et al., 2001; Cukierman et al., 2002). However, the previous studies have utilized a 3-D matrix in which the apical surface of the cell was not in contact with the matrix or cells embedded within the collagen were seeded into a plastic culture dish compared to the 3-D matrix in which the cells are completely embedded within and do not have interaction with any solid surface. This dissertation shows that focal adhesions and stress fibers do form in cells embedded within a 3-D collagen matrix in which the cells can only interact with themselves and the collagen. Differences in visualization of focal adhesions and stress fiber formation as previously reported may be due to differences in culturing and microscopy techniques.

I have shown that FAK is a critical protein in the generation and transmission of cellular force and may act to regulate tension through several potential mechanisms, including generation of a competent signaling complex, binding of the integrin to the underlying matrix, and/or linkage of the integrin to the actin cytoskeleton. FAK is a necessary component to elicit downstream signaling cascades following integrin engagement. After recruitment of FAK to the focal adhesions, phosphorylated FAK binds active Src, creating binding sites for other focal adhesion proteins and generating a kinase complex that can phosphorylate downstream components. However, I have shown that neither FAK nor Src kinase activity is necessary for 
the generation of cellular tension indicating that activity of the FAK-Src signaling complex is not critical for cellular force. Also, previous reports have shown that Pyk2 protein levels are increased following FAK knockout (Lim et al., 2008; Weis et al., 2008). This is thought to coincide with increases in p190RhoGEF expression and focal adhesion formation (Lim et al., 2008). The data presented here shows that Pyk2 protein levels were elevated in FAK knockout fibroblasts in 3-D, however, Pyk2 was unaltered in FAK knockdown endothelial cells. It is possible that elevated Pyk2 expression could alter Rho dynamics in the cell resulting in the changes in cellular tension generation. It would prove interesting to determine the effect of deletion of Pyk2 protein on tension generation to determine if elevation in Pyk2 expression with the loss of FAK is causing the defect in the ability to produce tension through alterations in cytoskeletal dynamics.

Since FAK localization to the focal adhesion is necessary for tension generation, FAK may also be responsible in promoting binding of the integrin to its matrix ligand. Talin binds to the $\beta$-integrin cytoplasmic tail and induces integrin activation by unmasking and disrupting the $\alpha$ - and $\beta$-integrin cytoplasmic tail interaction (Pfaff et al., 1998; Calderwood et al., 1999; Vinogradova et al., 2002; Tadokoro et al., 2003). This induces a conformational change in the extracellular domain of the integrins and promotes high-affinity ligand binding of the integrins (Vinogradova et al., 2002; Tadokoro et al., 2003). Given FAK's close proximity to the cell surface and the ability to bind to both talin and the $\beta$-integrin cytoplasmic tail, FAK may be involved in promoting the high affinity binding of the integrins to the underlying matrix allowing efficient linkage of the internal focal adhesion to the ECM. Also, following the stimulation of contractility, there is aggregation of integrins in the focal adhesions (Chrzanowska-Wodnicka and Burridge, 1996). FAK may be responsible for the aggregation of the integrins, resulting in 
the formation of focal adhesions in 3-D, since I did not observe classical focal adhesion formation in FAK knockout fibroblasts in 3-D. In addition, integrin recruitment to the focal adhesions and to the cell surface may be altered with the loss of FAK and does not allow proper linkage of the focal adhesions to the underlying matrix. I discovered that there was an alteration in integrin protein levels in FAK knockout fibroblasts compared to controls indicating that FAK may play a role in expression of integrin proteins. Therefore, FAK may be responsible for integrin engagement with the ECM and promoting high-affinity ligand binding to allow strong cellular adhesion formation in order to generate and transmit cellular force.

In order for the integrins to grip tightly to the ECM, the integrin cytoplasmic domains must be anchored to the actin cytoskeleton. This occurs through interaction of focal adhesion proteins with both the integrin and the actin stress fibers. Vinculin is a focal adhesion protein that can bind to talin or $\alpha$-actinin and is thought to be involved in anchoring F-actin to the cellular membrane (Zamir and Geiger, 2001; Pokutta and Weis, 2002; Cohen et al., 2006; Golji and Mofrad, 2013). I showed that in both endothelial cells and fibroblasts, the loss of FAK altered vinculin localization with an increase in vinculin-containing focal adhesion plaques in endothelial cells and diffuse vinculin localization without classical focal adhesion formation in FAK knockout fibroblasts. Therefore, FAK may be responsible for the proper recruitment and organization of vinculin within the focal adhesion hierarchy to allow vinculin to crosslink talin to the actin filaments and reinforce the focal adhesion. In addition, I observed that the actin cytoskeleton was altered with the loss of FAK with a lack of actin stress fiber formation in FAK knockout fibroblasts in 3-D but an increase in cortical distribution of actin filaments. Rho is responsible for stress fiber and focal adhesion formation and is involved in the activation of ROCK, which alters both myosin activity and cytoskeletal dynamics (Ridley and Hall, 1992; 
Hall, 1994; Takai et al., 1995; Amano et al., 1996; Chrzanowska-Wodnicka and Burridge, 1996). Katoh and colleagues (2007) showed that inhibition of ROCK disassembled central actin stress fibers and focal adhesions without affecting peripheral actin formation. Also, myosin phosphorylation actually precedes formation of stress fiber and focal adhesion formation and accompanied by an increase in cell contractility (Chrzanowska-Wodnicka and Burridge, 1996). I showed that with the loss of FAK in fibroblasts and endothelial cells there were alterations in cellular tension without any changes in myosin II phosphorylation. It was previously shown that in 2-D, loss of FAK leads to a deregulation in Rho and ROCK dynamics (Ren et al., 2000; Chen et al., 2002; Pirone et al., 2006). Thus, the loss of FAK in 3-D may lead to a loss in Rho and ROCK activation, resulting in changes in the actin cytoskeleton and focal adhesion formation, leading to alteration in cellular tension. Therefore, determining Rho-GTP levels and activity of ROCK following the loss of FAK would be necessary to determine the involvement of FAK in the regulation of Rho-mediated cellular tension generation.

The finding that FAK is a critical protein in the regulation of cellular force can have many implications in the study of human disease. Increases in vascular permeability and the development of edema occur during inflammation and may be due in part to disruption of cell cohesion and changes in intrinsic contractile activity of endothelial cells (Lucas et al., 2009; Dushianthan et al., 2011; Mac et al., 2011). Given the importance of FAK in regulating endothelial cell tension and monolayer barrier, inhibition of FAK function in endothelial cells could aid in blocking permeability increases during the inflammatory process and lessen edema formation. Also, FAK may be important in blocking the progression of cancer. FAK expression has been found to be elevated in certain types of cancer (Owens, 1995). Enhanced expression of FAK could lead to increased force generation and remodeling of the ECM to produce a more 
rigid matrix. It has been shown that increased tumor matrix rigidity leads to cellular proliferation and tumor growth (Tilghman, 2010). Therefore, reduction in tumor specific FAK protein levels could reduce tumor cell proliferation and block tumor growth.

Fibroblasts are important cells within the body that are involved in the secretion and organization of matrix proteins. Pulmonary fibrosis occurs due to the formation of excess fibrous connective tissue in the lungs, which is mediated by fibroblasts. It has been shown previously that FAK expression is elevated in fibroblasts isolated from pulmonary fibrosis patients and inhibition of FAK prevents the formation of pulmonary fibrosis (Lagares, 2012). Enhanced FAK expression could lead to abnormal matrix secretion and organization leading to the formation of fibrosis. Reduction of FAK protein levels in lung fibroblasts could prevent the accumulation of matrix proteins and slow the progression of pulmonary fibrosis. Fibroblasts are also important in wound healing, and cellular force and matrix organization plays a large role in the closure of a wound. Postoperative adhesions are fibrous bands of abnormal internal scar tissue that join tissue surfaces that are not usually connected and can occur in any area of the human body that sustains injury or undergoes surgery. Although usually temporary, if these adhesions are not properly dissolved through digestion by fibrinolytic enzymes, macrophages and fibroblasts are recruited to the site and lay down matrix proteins forming a more permanent fibrous adhesion, which can prevent muscles and organs from moving freely and even twisting and pulling organs from their normal positions, resulting in severe pain, impaired organ function, and/or restriction of motion. The remodeling of the ECM and cell contraction is critical for proper wound healing. A previous study of skin fibrosis and hyptertrophic scar formation has shown that mechanical force causes the secretion of monocyte chemoattractant protein 1 (MCP1) and collagen production in a FAK-mediated process (Wong et al., 2011). Therefore, 
uncoupling mechanical tension from fibrosis formation may prove successful in preventing abnormal wound healing. Given FAK's kinase-independent involvement in cellular tension generation and collagen matrix organization in fibroblasts, developing treatments that locally alter FAK localization to focal adhesions in fibroblasts may protect against aberrant scar tissue formation and block the formation of post surgical adhesions.

In conclusion, the data presented here shows that FAK is an integral part in nonmuscle cellular tension. While many studies have shown FAK to be a necessary component of contractile signaling pathways, very few studies have attempted to determine FAK's direct role in the generation and transmission of adhesive forces. The investigation of nonmuscle cellular tension generation in 3-D shows a novel role for FAK in cell contraction. Initially, the primary function of FAK was discovered to be in the transmission of intercellular signals following integrin engagement via phosphorylation of downstream effectors in order to influence cellular behavior. However, data presented here shows that FAK localization to focal adhesions in fibroblasts is critical for tension generation without a need for kinase activity suggesting that FAK can function as a structural protein within the adhesion to stabilize adhesions and allow the cell to contract. While studying FAK function in 2-D tissue culture dishes is necessary, the utilization of 3-D culture allows the determination of FAK's involvement in cell contraction through direct measurement of cellular tension and results can be extrapolated to FAK function in vivo. Alterations in cell contractility and cell migration play key roles in the development of certain diseases including developmental disorders, Crohn's disease, atherosclerosis, and cancer (Raines, 2000; McManus and Golden, 2005; Brand et al., 2006; Chaffer and Weinberg, 2011). Thus, FAK may be a novel target in many cell types for the development of treatments to alter cell contractility and block disease progression. 


\section{References}

Amano, M., M. Ito, K. Kimura, Y. Fukata, K. Chihara, T. Nakano, Y. Matsuura, and K. Kaibuchi. 1996a. Phosphorylation and activation of myosin by Rho-associated kinase (Rhokinase). J. Biol. Chem. 271:20246-20249.

Brand, S., F. Beigel, T. Olszak, K. Zitzmann, S.T. Eichhorst, J-M. Otte, H. Diepolder, A. Marquardt, W. Jagla, A. Popp, S. Leclair, K. Hermann, J. Seidere, T. Ochsenkuhn, B. Goke, C.J. Auernhammer, and J. Dambacher. 2006. IL-22 is increased in active Crohn's disease and promotes proinflammatory gene expression and intestinal epithelial cell migration. Am.J. Physiol. Gastrointest. Liver Physiol. 290:G827-G838.

Calderwood, D.A., R. Zent, R. Grant, D.J. Rees, R.O. Hynes, and M.H. Ginsberg. 1999. The Talin head domain binds to integrin beta subunit cytoplasmic tails and regulates integrin activation. J. Biol. Chem. 274:28071-28074.

Chaffer, C.L., and R.A. Weinberg. 2011. A perspective on cancer cell metastasis. Science. 331:1559-1564.

Chen, B.H., J.T. Tzen, A.R. Bresnick, and H.C. Chen. 2002. Roles of Rho-associated kinase and myosin light chain kinase in morphological and migratory defects of focal adhesion kinasenull cells. J. Biol. Chem. 277:33857-33863.

Chrzanowska-Wodnicka, M., and K. Burridge. 1996. Rho-stimulated contractility drives the formation of stress fibers and focal adhesions. J. Cell Biol. 133:1403-1415.

Clemente, C.F., T.F. Tornatore, T.H. Theizen, A.C. Deckmann, T.C. Pereira, I. Lopes-Cendes, J.R. Souza, and K.G. Franchini. 2007. Targeting focal adhesion kinase with small interfering RNA prevents and reverses load-induced cardiac hypertrophy in mice. Circ Res. 101:13391348.

Cohen, D.M., B. Kutscher, H. Chen, D.B. Murphy, and S.W. Craig. 2006. A conformational switch in vinculin drives formation and dynamics of a talin-vinculin complex at focal adhesions. J. Biol. Chem. 281:16006-16015.

Cukierman, E., R. Pankov, D.R. Stevens, and K.M. Yamada. 2001. Taking cell-matrix adhesions to the third dimension. Science. 294:1708-1712.

Cukierman, E., R. Pankov, and K.M. Yamada. 2002. Cell interactions with three-dimensional matrices. Curr. Opin. Cell Biol. 14:633-639.

Dushianthan, A., M.P. Grocott, A.D. Postle, and R. Cusack. 2011. Acute respiratory distress syndrome and acute lung injury. Postgrad. Med. J. 87:612-622.

Elsdale, T., and J. Bard. 1972. Collagen substrata for studies on cell behavior. J. Cell Biol. 54:626-637. 
Fraley, S.I., Y. Feng, R. Krishnamurthy, D-H. Kim, A. Celedon, G.D. Longmore, and Wirtz, D. 2010. A distinctive role for focal adhesion proteins in three-dimensional cell motility. Nat. Cell Biol. 12:598-604.

Friedl, P., and E.B. Brocker. 2000. The biology of cell locomotion within three-dimensional extracellular matrix. Cell Mol. Life Sci. 57:41-64.

Friedl, P., F. Entschladen, C. Conrad, B. Niggeman, and K.S. Zanker. 1998. CD4+ T lymphocytes migrating in three-dimensional collagen lattices lack focal adhesions and utilize $\beta 1$ integrin-independent strategies for polarization, interaction with collagen fibers and locomotion. Eur. J. Immunol. 28:2331-2343.

Gabarra-Niecko, V., M.D. Schaller, and J.M. Dunty. 2003. FAK regulates biological processes important for the pathogenesis of cancer. Cancer Metastasis Rev. 22:359-374.

Golji, J., and M.R. Mofrad. 2013. The interaction of vinculin with actin. PLoS Comput. Biol. 9:e1002995.

Guan, J-L., and D. Shalloway. 1992. Regulation of focal adhesion-associated protein tyrosine kinase by both cellular adhesion and oncogenic transformation. Nature. 358:690-692.

Guan, J-L., J.E. Trevithick, and R.O. Hynes. 1991. Fibronectin/integrin interaction induces tyrosine phosphorylation of a 120-kDa protein. Cell Regulation. 2:951-964.

Hall, A., 1994. Small GTP-binding proteins and the regulation of the actin cytoskeleton. Annu. Rev. Cell Biol. 10:31-54.

Hanks, S.K., M.B. Calalb, M.C. Harper, and S.K. Patel. 1992. Focal adhesion protein-tyrosine kinase phosphorylated in response to cell attachment to fibronectin. Proc. Natl. Acad. Sci. USA. 89:8487-8491.

Ilic, D., Y. Furuta, S. Kanazawa, N. Takeda, K. Sobue, N. Nakatsuji, S. Nomura, J. Fujimoto, M. Okada, and T. Yamamoto. 1995. Reduced cell motility and enhanced focal adhesion contact formation in cells from FAK-deficient mice. Nature. 377:539-544.

Katoh, K., Y. Kano, and S. Ookawara. 2007. Rho-kinase dependent organization of stress fibers and focal adhesions in cultured fibroblasts. Genes Cells. 12:623-638.

Knight, B., C. Laukaitis, N. Akhtar, N.A. Hotchin, M. Edlund, and A.R. Horwitz. 2000.

Visualizing muscle cell migration in situ. Curr. Biol. 10:576-585.

Kornberg, L.J., H.S. Earp, J.T. Parsons, M. Schaller, and R.L. Juliano. 1992. Cell adhesion or integrin clustering increases phosphorylation of a focal adhesion-associated tyrosine kinase. $J$. Biol. Chem. 267:23439-23442. 
Kornberg, L.J., H.S. Earp, C.E. Turner, C. Prockop, and R.L. Juliano. 1991. Signal transduction by integrins: increased protein tyrosine phosphorylation caused by clustering of $\beta_{1}$ integrins.

Proc. Natl. Acad. Sci. USA. 88:8392-8396.

Kubow, K.E., and A.R. Horwitz. 2011. Reducing background fluorescence reveals adhesions in 3D matrices. Nat. Cell Biol. 13:3-5.

Lagares, D., O. Busnadiego, R.A. Garcia-Fernandez, M. Kapoor, S. Liu, D.E. Carter, D. Abraham, X. Shi-Wen, P. Carreira, B.A. Fontaine, B.S. Shea, A.M. Tager, A. Leask, S. Lamas, and F. Rodriguez-Pascual. 2012. Inhibition of focal adhesion kinase prevents experimental lung fibrosis and myofibroblast formation. Arthritis Rheum. 64:1653-1654.

Lim, Y., S.T. Lim, A. Tomar, M. Gardel, J.A. Bernard-Trifilo, X.L. Chen, S.A. Uryu, R. CaneteSoler, J. Zhai, H. Lin, W.W. Schlaepfer, P. Nalbant, G. Bokoch, D. Ilic, C. Waterman-Storer, and D.D. Schlaepfer. 2008. PyK2 and FAK connections to p190Rho guanine nucleotide exchange factor regulate RhoA activity, focal adhesion formation, and cell motility. J. Cell Biol. 180:187-203.

Lucas, R., A.D. Verin, S.M. Black, and J.D. Catravas. 2009. Regulators of endothelial and epithelial barrier integrity and function in acute lung injury. Biochem. Pharmacol. 77:1763:1772.

Mac, S.R., D.F. McAuley, and M.A. Matthay. 2011. Acute lung failure. Semin. Respir. Crit. Care Med. 32:607-625.

McManus, M.F., and J.A. Golden. 2005. Neuronal migration in developmental disorders. J. Child Neurol. 20:280-286.

Owens, L.V., L. Xu, R.J. Craven, G.A. Dent, T.M. Weiner, L. Kornberg, E.T. Liu, and W.G. Cance. 1995. Overexpression of the focal adhesion kinase (p125FAK) in invasive human tumors. Cancer Res. 55:2752-2755.

Peng, X., X. Wu, J.E. Druso, H. Wei, A.Y. Park, M.S. Kraus, A. Alcaraz, J. Chen, S. Chien, R.A. Cerione, and J.L. Guan. 2008. Cardiac developmental defects and eccentric right ventricular hypertrophy in cardiomyocyte focal adhesion kinase (FAK) conditional knockout mice. Proc. Natl. Acad. Sci. USA. 105:6638-6643.

Petroll, W.M., L. Ma, and J.V. Jester. 2003. Direct correlation of collagen matrix deformation with focal adhesion dynamics in living corneal fibroblasts. J. Cell Sci. 116:1481-1491.

Pfaff, M., S. Liu, D.J. Erle, and M.H. Ginsberg. 1998. Integrin beta cytoplasmic domains differentially bind to cytoskeletal proteins. J. Biol. Chem. 273:6104-6109.

Pirone, D.M., W.F. Liu, S.A. Ruiz, L. Gao, S. Raghaven, C.A. Lemmon, L.H. Romer, and C.S. Chen. 2006. An inhibitory role for FAK in regulating proliferation: a link between limited adhesion and RhoA-ROCK signaling. J. Cell Biol. 174:277-288. 
Pokutta, S., and W.I. Weis. 2002. The cytoplasmic face of cell contacts. Curr. Opin. Struct. Biol. 12:255-262.

Raines, E.W. 2000. The extraceullar matrix can regulate vascular cell migration, proliferation, and survival: relationships to vascular disease. Internatl. J. Exp. Pathology. 81:173-182.

Ren, X.D., W.B. Kiosses, D.J. Sieg, C.A. Otey, D.D. Schlaepfer, and M.A. Schwartz. 2000. Focal adhesion kinase suppresses Rho activity to promote focal adhesion turnover. J. Cell Sci. 113:3673-3678.

Ridley A.J., and A. Hall. 1992. The small GTP-binding protein rho regulates the assembly of focal adhesions and actin stress fibers in response to growth factors. Cell. 70:389-399.

Ruoslahti, E. 1991. Integrins. J. Clin. Invest. 87:1-5.

Roskelley, C.D., and M.J. Bissell. 1995. Dynamic reciprocity revisited: a continuous, bidirectional flow of information between cells and the extracellular matrix regulates mammary epithelial cell function. Biochem. Cell Biol. 73:391

Schaller, M.D., C.A. Borgman, B.S. Cobb, R.R. Vines, A.B. Reynolds, and J.T. Parsons. 1992. pp125FAK, a structurally distinctive protein-tyrosine kinase associated with focal adhesions. Proc. Natl. Acad. Sci. USA. 89:5192-5196.

Schnitzer, J.E., A. Siflinger-Birnboim, P.J. Del Vecchio, and A.B. Malik. 1994. Segmental differentiation of permeability, protein glycosylation, and morphology of cultured bovine lung vascular endothelium. Biochem. Biophys. Res. Commun. 199:11-19.

Sieg, D.J., C.R. Hauck, D. Ilic, C.K. Klingbeil, E. Schaefer, C.H. Damsky, and D.D. Schlaepfer. 2000. FAK integrates growth-factor and integrin signals to promote cell migration. Nat. Cell Biol. 2:249-256.

Sieg, D.J., C.R. Hauck, and D.D. Schlaepfer. 1999. Required role of focal adhesion kinase (FAK) for integrin-stimulated cell migration. J. Cell Sci. 112:2677-2691.

Tadokoro, S., S.J. Shattil, K. Eto, V. Tai, R.C. Liddington, J.M. de Pereda, M.H. Ginsberg, and D.A. Calderwood. 2003. Talin binding to integrin beta tails: a final common step in integrin activation. Science. 302:103-106.

Takai, Y., T. Sasaki, K. Tanaka, and H. Nakanishi. 1995. Rho as a regulator of the cytoskeleton. Trends Biochem. Sci. 20:227-231.

Tilghman, R.W., C.R. Cowan, J.D. Mih, Y. Koryakina, D. Gioeli, J.K. Slack-Davis, B.R. Blackman, D.J. Tschumperlin, and J.T. Parsons. 2010. Matrix rigidity regulates cancer cell growth and cellular phenotype. PLoS One. 5:e12905. 
van Nimwegen, M.J., S. Verkoeijen, L. van Buren, D. Burg, and B. van de Water. 2005. Requirement for focal adhesion kinase in the early phase of mammary adenocarcinoma lung metastasis formation. Cancer Res. 65:4698-4706.

Vinogradova, O., A. Velyvis, A. Velyviene, B. Hu, T. Haas, E. Plow, and J. Qin. 2002. A structurally mechanism of integrin alpha(IIb)beta(3) "inside-out" activation as regulated by its cytoplasmic face. Cell. 110:587-597.

Weis, S.M., S.T. Lim, K.M. Lutu-Fuga, L.A. Barnes, X.L. Chen, J.R. Gothert, T.L. Shen, J.L. Guan, D.D. Schlaepfer, and D.A. Cheresh. 2008. Compensatory role for Pyk2 during angiogenesis in adult mice lacking endothelial cell FAK. J. Cell Biol. 181:43-50.

Wong, V.W., K.C. Rustad, S. Akaishi, M. Sorkin, J.P. Glotzbach, M. Januszyk, E.R. Nelson, K. Levi, J. Paterno, I.N. Vial, A.A. Kuang, M.T. Longaker, and G.C. Gurtner. 2011. Focal adhesion kinase links mechanical force to skin fibrosis via inflammatory signaling. Nat. Med. 18:148-152.

Zamir, E., and B. Geiger. 2001. Molecular complexity and dynamics of cell-matrix adhesions. J. Cell Sci. 114(Pt 20):3583-3590. 
Kimberly (Miede) Arnold

48 Brier Avenue Wilmington, DE 19805

(410) 982-9123 kamiede@gmail.com

\section{EDUCATION}

Ph.D., Biomedical Sciences

June 2013

Cellular \& Integrative Physiology program

West Virginia University School of Medicine

Morgantown, WV

Dissertation title: The role of focal adhesion kinase in nonmuscle cell contraction

Advisor: Robert B. Wysolmerski, Ph.D.

Committee members: Dr. Jun Liu, Dr. Karen Martin, Dr. Fred Minnear, Dr. Michael

Schaller

Bachelor of Science in Genetic Engineering

Cedar Crest College

May 2005

Thesis title: Bacteriophage codon usage reflects host codon usage

Allentown, PA

Advisor: Richard Kliman, Ph.D.

\section{RESEARCH EXPERIENCE:}

Postdoctoral Researcher

College of Health Sciences, University of Delaware

Advisors: Daniel Flynn, Ph.D. and Jennifer Sims-Mourtada, Ph.D.

July 2013-Present

Newark, DE

Graduate Research Assistant

July 2006-June 2013

Biomedical Sciences, West Virginia University School of Medicine Morgantown, WV Advisor: Robert Wysolmerski, Ph.D.

- Showed focal adhesion kinase (FAK) is an essential protein for cellular tension generation through measurement of tension production by cells within a threedimensional matrix

- Determined FAK is important in reorganizing the extracellular matrix by imaging a three-dimensional collagen matrix with two-photon and scanning electron microscopy

- Developed method to image cell adhesions in three-dimensional matrix

Independent Undergraduate Research

Department of Biology, Cedar Crest College
January 2003-May 2005

Allentown, PA

Project title: Investigating Codon Bias in Bacteria and Respective Bacteriophage Genomes

Advisor: Richard Kliman, Ph.D.

- Examined the codon usage in the genome of bacteria and their respective infecting bacteriophage to determine if phage are related to their host

- Employed the use of statistical analysis and genomic analysis using high capacity computers 
Project title: Investigating Codon Bias in Bacteria and Respective Bacteriophage Advisor: Richard Kliman, Ph.D.

- Determined the correlation between bacteriophage and host codon usage

Freshman Research Director

Department of Biology, Cedar Crest College

Project tile: Codon Bias Among Eukaroytes and Prokaryotes

- Managed and designed an independent research project for a freshman biology student

\section{Freshman Research}

January 2002-May 2002

Department of Biology, Cedar Crest College

Allentown, PA

Project title: Sequencing the ORF-314 Gene In Janthinobacterium lividum Infecting Bacteriophage

- Isolated and sequenced the ORF-314 tail fiber gene of the bacteriophage to examine evolutionary relationships among phage

\section{RESEARCH SKILLS}

- Tissue culture techniques: growth and maintenance of mammalian cell lines (including human) in two- and three-dimensional culture, transfection, and development of stable cell lines through lentiviral infection

- Immunocytochemistry \& cellular imaging techniques: use of confocal, live cell, and two-photon microscopy

- Molecular biology techniques: subcloning, PCR, agarose gel electrophoresis, and DNA extraction

- Microbial culture techniques: growth and maintenance of $E$. coli in liquid and solid media, and transformation

- Viral techniques: large scale amplification and purification of adenovirus and lentivirus

- Protein techniques: western immunoblotting

\section{PUBLICATIONS}

Arnold, K.M., Goeckeler, Z.M., Galvin, N., and R.B. Wysolmerski. Focal adhesion kinase knockout alters isometric tension generation and extracelluar matrix organization. (Manuscript to be submitted September 2013) 
Arnold, K.M., Goeckeler, Z.M., and R.B. Wysolmerski. 2013. Loss of focal adhesion kinase enhances endothelial barrier function and increases focal adhesions. Microcirculation. doi: 10.1111/micc.12063.

\section{TEACHING EXPERIENCE}

Teaching Assistant, Medical Student Level course

Human Function: CCMD 730

August 2007-December 2007

West Virginia University School of Medicine Morgantown, WV

- Facilitated a small group of first year medical students in answering physiology related questions

Genetics, Freshman Chemistry, \& Introduction to Cell Biology Tutor Academic Services, Cedar Crest College

August 2002-May 2005 Allentown, PA

- Assisted students with homework problems, understanding concepts, and studying for upcoming tests and quizzes

\section{PRESENTATIONS}

Arnold, K.M. and R. Wysolmerski. May 2011. Role of focal adhesion kinase in cell contractility. Presented for Julie Betschart Symposium, Department of Physiology \& Pharmacology, West Virginia University, Morgantown, WV (Award for first place).

Arnold, K.M. and R. Wysolmerski. January 2011. Involvement of focal adhesion kinase in vascular permeability. Presented for Department of Physiology Seminar, West Virginia University, Morgantown, WV.

Miede, K. and R. Wysolmerski. February 2010. Focal adhesion kinase and endothelial cell tension. Presented for Department of Physiology Seminar, West Virginia University, Morgantown, WV.

Miede, K. and R. Wysolmerski. September 2009. Focal adhesion kinase and endothelial cell isometric tension. Presented at Cardiovascular and Respiratory Sciences Center Work in Progress meeting, West Virginia University, Morgantown, WV.

Miede, K. and R. Wysolmerski. April 2009. Focal adhesion kinase and endothelial cell isometric tension. Presented at cytoskeletal research interest group meeting, West Virginia University, Morgantown, WV.

Miede, K. and R. Wysolmerski. May 2007. Integrin-linked kinase (ILK) and endothelial cell isometric tension. Presented for Department of Physiology Seminar, West Virginia University, Morgantown, WV. 


\section{POSTERS}

Arnold, K.M., Goeckeler, Z.M., Galvin, N., and R.B. Wysolmerski. 2012. Role of focal adhesion kinase in isometric tension generation and extracellular matrix organization. Molecular Biology of the Cell 23:24 (suppl), Abstract \#2128, The American Society for Cell Biology, San Francisco, CA.

Arnold, K.M., Goeckeler, Z.M., Galvin, N., and R.B. Wysolmerski. 2012. Role of focal adhesion kinase in isometric tension generation and extracellular matrix organization. Appalachain Regional Cell Conference, Charleston, WV.

Arnold, K.M., Goeckeler, Z., and R. Wysolmerski. 2011. FAK knockdown alters endothelial cell monolayer integrity without changing isometric tension. FASEB J. 25, 820.22 Experimental Biology, Washington, DC.

Arnold, K., Goeckeler, Z., and R. Wysolmerski. 2011. FAK knockdown alters endothelial cell monolayer integrity without changing isometric tension. E. J. Van Liere Memorial Research Convocation, Morgantown, WV. (Award for first place poster, Basic Science 2005)

Miede, K., Goeckeler, Z., Pumiglia, K., and R. Wysolmerski. 2010. FAK and endothelial cell isometric tension. FASEB J. 24, lb423 Experimental Biology, Anaheim, CA

Miede, K., Goeckeler, Z., and R. Wysolmerski. 2010. FAK and endothelial cell isometric tension. E.J. Van Liere Memorial Research Convocation, Morgantown, WV.

Miede, K., Goeckeler, Z., and R. Wysolmerski. 2009. Focal adhesion kinase and isometric tension. E. J. Van Liere Memorial Research Convocation, Morgantown, WV.

Miede, K., Goeckeler, Z., and R. Wysolmerski. 2008. Involvement of integrin-linked kinase in endothelial cell isometric tension. CoBRE-InBRE meeting, Morgantown, WV.

Miede, K., Goeckeler, Z., and R. Wysolmerski. 2008. Involvement of integrin-linked kinase in endothelial cell isometric tension. E. J. Van Liere Memorial Research Convocation, Morgantown, WV.

Kliman, R.M. and K.A. Miede. 2007. Coevolution of bacteriophage and host codon and amino acid usage. Lehigh Valley Ecology and Evolution Society, Center Valley, PA.

Miede, K.A. and R.M. Kliman. 2005. Bacteriophage codon usage reflects host codon usage. Pennsylvania Academy of Science, Camp Hill, PA.

Miede, K.A. and R.M. Kliman. 2005. Bacteriophage codon usage reflects host codon usage. Northeast Ecology and Evolution Conference, State College, PA. 
Miede, K.A. and R.M. Kliman. 2004. Bacteriophage codon usage reflects host codon usage. Society for the Study of Evolution, Fort Collins, CO.

Miede, K.A. and R.M. Kliman. 2004. Correspondence between phage and host codon usage. Lehigh Valley Ecology and Evolution Society, Allentown, PA.

Miede, K.A. and R.M. Kliman. 2004. Correspondence between phage and host codon usage. Pennsylvania Academy of Science, Pittsburgh, PA.

Miede, K.A. and R.M. Kliman. 2003. Correspondence between phage and host codon usage. New England Molecular Evolutionary Biologists, Storrs, CT. (Prize for best undergraduate poster).

\section{AWARDS \& FUNDING:}

- Travel Award; West Virginia University

December 2012 Biomedical Sciences Research Office

- First place oral presentation at Julie Betschart Symposium

Department of Physiology \& Pharmacology, West Virginia University

May 2011

- First place poster presentation at E.J. Van Liere Memorial Research Convocation Department of Physiology \& Pharmacology, West Virginia University May 2011

- $\underline{\text { Predoctoral Training Grant Awardee }}$

NIH Cardiovascular and Pulmonary Disease T-32 Training Grant July 2008-June 2011

- Golden Key International Honour Society

November 2008

- Beta Beta Beta/Department of Biological Sciences Award

May 2005

- Delphi, Cedar Crest College Honor Society

May 2004

- Beta Beta Beta, National Honor Society for Biology

May 2003 (Chapter president 2004-05)

\section{PROFESSIONAL MEMBERSHIPS}

The American Society for Cell Biology

August 2012-Present

\section{PROFESSIONAL DEVELOPMENT WORKSHOPS}

Grant Writing Seminar

June 2011

West Virginia University Morgantown, WV

Preparing Future Faculty Workshop

May 2010

West Virginia University Morgantown, WV 